\title{
Modelling and optimizing through-the-Earth radio transmissions
}

\author{
by \\ Maxim Ralchenko \\ A thesis submitted to the \\ Faculty of Graduate and Postdoctoral Affairs \\ in partial fulfillment of the requirements for the degree of
}

Doctor of Philosophy in Earth Sciences

Department of Earth Sciences

Carleton University

Ottawa, Ontario

2017

(C)Copyright

Maxim Ralchenko, 2017 


\section{Abstract}

Through-the-Earth (TTE) radio has been proposed for emergency communications in locations inaccessible by conventional means, such as underground mines. While the technology is viable, it is unclear how the signal propagates in inhomogeneous media; neither modelling or obtaining a conductivity distribution in the context of TTE radio has been previously attempted. With a robust model, many practical questions can be answered, such as what is the maximum range or the optimal frequency to use, or where the transmitter and receiver should be ideally placed. To this end, a finitedifference time-domain (FDTD) code was developed and optimized for the forward modelling of TTE radio transmissions. This method is computationally intensive, and to improve performance, it was run on a graphics processing unit (GPU). The code was validated against analytical solutions for simple geometries.

The use of a TTE radio signal to estimate the conductivity structure of an operating environment was demonstrated at an experimental coal mine. A transmitter was set up on the surface, and the signal strength in the underground mine was measured at several locations and frequencies. A four-layer conductivity model, with topography, was fitted to this data, using the FDTD code.

TTE radio signal range has been experimentally observed to increase in the presence of elongated conductors. A thin-wire approximation was added to the FDTD code, and conductivity models were fitted to match observed data for propagation 
along elevator shafts in multistory buildings and along railway tracks. Multistory buildings present a separate challenge due to their conductive reinforced concrete structure. By representing the floors and walls as thin sheets of a given conductance, observed and modelled data were reconciled for a variety of buildings, including aboveground reinforced concrete constructions, a building with steel floors, and a building partially below ground, and for placements of the transmitter and receiver inside or outside the building.

Further work remains in modelling a wider variety of settings where TTE radio could be used, such as buildings with different materials. Another avenue of research is to improve the performance of the modelling code, by refining the numerical method used. 


\section{Acknowledgments}

This thesis could not have been done without the contributions of many people.

First, I would like to thank my supervisor, Prof. Claire Samson, for her invaluable advice for not only during this thesis, but over my whole scientific career since the summer after my second year during my undegraduate studies.

I thank Vital Alert Communication, Inc., for their valuable financial support, via the Industrial Postgraduate Scholarship program with the Natural Sciences and Engineering Research Council (NSERC) over the course of my research. Mr. Markus

Svilans provided extended advice and guidance during my $\mathrm{PhD}$, particularly during the first half. Mr. Mike Roper also provided considerable input over the course of the $\mathrm{PhD}$ thesis, especially during the latter half. Mr. Michael Homer is thanked for proofreading and giving his blessing to submit all the papers and abstracts, as well as for his assistance in the field. Mr. Vladimir Puzakov is thanked for his help with the hardware and for assistance in the field. Mr. Brian Du is thanked for his assistance in the field. Dr. Peter Kwasniok is thanked for his input into the numerous experiments done over the past three years. Mr. Andrew Schenk is thanked for his assistance with the hardware. Mr. Andrew Wahl is thanked for his logistical assistance. Mr. John Steckley, of Innovative Wireless Techologies, is thanked for his assistance in the field, during a trip to West Virginia demonstrating IWT and Vital Alert products.

The committee for my comprehensive examination, Prof. Dariush Motazedian 
(Carleton Univesity), Prof. Pascal Audet (University of Ottawa), Prof. Michel Chouteau (École Polytechnique), and Dr. Richard Ernst (Carleton University and Ernst Geosciences), provided valuable advice to guide my research. I further thank Prof. Chouteau, as well as Dr. Randy Enkin (Natural Resources Canada) for the discussions that led to the second paper in the integrated thesis. I further thank my thesis examination committee, Prof. Heather Logan (Carleton University), Prof. James Macnae (RMIT University), Prof. Andy Adler (Carleton University), Prof. Audet, and Prof. Chouteau, for their input.

I thank Alpha Natural Resources and the National Institute of Occupational Safety and Health (NIOSH) for giving access to their mines. Furthermore, I thank Mr. Nick Damiano and Mr. Bruce Whisner of NIOSH during the visit to NIOSH's Safety Research Coal Mine.

I am grateful for the assistance in the field from Mr. Andrew Hay and Mr. Peter Oliver, both from Carleton University.

The input of anonymous reviewers resulted in improvements to the manuscripts. Last but not least, I wish to thank Ms. Carling Walsh, who has done everything between proofreading papers, helping in the field, to being a co-author. 


\section{Preface}

This document is an integrated thesis consisting of four articles published, accepted, or submitted to peer-reviewed scientific journals on the topic of modelling and optimizing through-the-Earth (TTE) radio transmissions:

1. Ralchenko, M., M. Svilans, C. Samson, and M. Roper, 2015, Finite-difference time-domain modelling of through-the-Earth radio signal propagation: Computers \& Geosciences, 85, 184-195. [Chapter 3]

2. Ralchenko, M., M. Svilans, C. Samson, C. Walsh, and M. Roper, 2017, Estimating subsurface conductivity while optimizing through-the-Earth radio communications using a wideband electromagnetic signal: accepted in Geophysics. [Chapter 4]

3. Ralchenko, M., M. Roper, M. Svilans, and C. Samson, 2017, Coupling of very low frequency through-the-Earth radio signals to elongated conductors: accepted in IEEE Transactions on Antennas and Propagation. [Chapter 5]

4. Ralchenko, M., M. Roper, C. Samson, and M. Svilans, 2017, Near-field VLF electromagnetic signal propagation in multistory buildings: submitted to IEEE Transactions on Antennas and Propagation. [Chapter 6]

Each article is reproduced in whole. Table and figure numbers, as well as reference formatting, has been standardized and updated to be consistent within the thesis. 
In paper 1, M. Ralchenko wrote the manuscript, was the lead software developer, and performed and analyzed all the simulations. M. Svilans assisted with the development of the code. M. Svilans and M. Roper provided guidance during the development of the software and provided comments on the article. C. Samson provided extensive comments on the article.

In paper 2, M. Ralchenko wrote the manuscript, developed and ran the field procedures, and developed and ran analysis code. In paper 2, M. Svilans provided guidance during the development of the field procedure, participated in the field work, developed the data processing routines for reducing the raw field data to stacked and filtered waveforms, and reduced the raw field data. C. Walsh contributed a 2-D and 3-D map describing the field work. M. Svilans and M. Roper provided comments on the article. C. Samson provided extensive comments on the manuscript and helped to address the reviewers' comments.

In paper 3, M. Ralchenko wrote the manuscript, developed the software, and performed the data analysis. M. Ralchenko and M. Roper developed and refined the field procedure and gathered field data. C. Samson provided extensive comments on the article. M. Svilans gathered field data and provided comments on the article.

In paper 4, M. Ralchenko wrote the manuscript, developed the simulaton procedure, and performed the data analysis. M. Ralchenko and M. Roper developed and refined the field procedure and gathered field data. C. Samson provided extensive comments on the article. M. Roper and M. Svilans provided additional comments on the article.

The thesis supervisor, Prof. Claire Samson, acknowledges the above information as accurate. 


\section{Table of Contents}

$\begin{array}{lll}\text { Abstract } & \text { ii }\end{array}$

Acknowledgments $\quad$ iv

Preface vi

Table of Contents viii

List of Tables $\quad$ xii

List of Figures $\quad$ xiii

1 Introduction 1

1.1 Through-the-Earth radio . . . . . . . . . . . . . . . . . . . 1

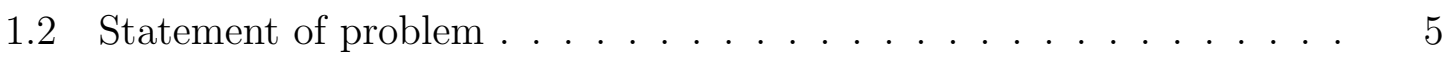

1.3 Objectives and originality $\ldots \ldots \ldots \ldots \ldots \ldots \ldots$

2 Methodology $\quad 9$

2.1 Overview . . . . . . . . . . . . . . . . . . . 9

2.2 Finite-difference time-domain method . . . . . . . . . . . . . . 10

2.3 Through-the-Earth radio equipment . . . . . . . . . . . . . . . 12

3 FDTD modelling of TTER signal propagation 14 
3.1 Abstract . . . . . . . . . . . . . . . . . . . 14

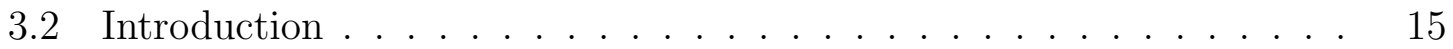

3.3 Literature review $\ldots \ldots \ldots \ldots \ldots$

3.4 Methods . . . . . . . . . . . . . . . . . . . . . . . . 19

3.4.1 Finite-difference time-domain method . . . . . . . . . . . . 19

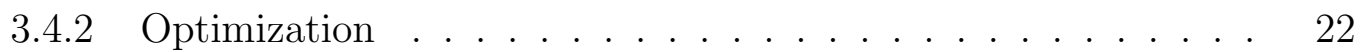

3.5 Results and discussion $\ldots \ldots \ldots \ldots \ldots$

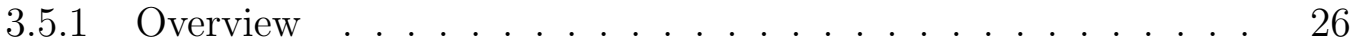

3.5 .2 Case A: Biot-Savart law . . . . . . . . . . . . . . . 29

3.5.3 Case B: Homogeneous halfspace . . . . . . . . . . . . . . 32

3.5 .4 Case C: Layered Earth . . . . . . . . . . . . . . . . 33

3.5.5 Case D: Faulted coal seam . . . . . . . . . . . . . . 35

3.5 .6 Case E: Salt diapir . . . . . . . . . . . . . . . . . . . . 38

3.6 Conclusion . . . . . . . . . . . . . . . . . . . . . . . . . . . . . . . 41

3.7 Acknowledgments . . . . . . . . . . . . . . . . . . . . . . . . . . 42

3.8 Appendix I: Homogeneous halfspace . . . . . . . . . . . . . . . 43

3.9 Appendix II: Three-layer Earth . . . . . . . . . . . . . . . . . 44

4 Estimating subsurface conductivity 46

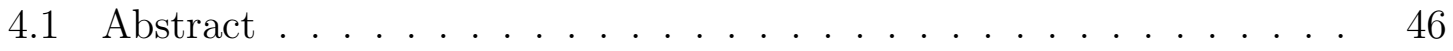

4.2 Introduction and objective $\ldots \ldots \ldots \ldots \ldots \ldots$

4.3 Literature review . . . . . . . . . . . . . . . . . . . . 48

4.3 .1 Electromagnetic methods . . . . . . . . . . . . . . . 48

4.3 .2 Radio imaging method . . . . . . . . . . . . . . . . 49

4.3 .3 Transmission tests . . . . . . . . . . . . . . . . . 49

4.4 Theoretical background . . . . . . . . . . . . . . . . 52 
4.5 Proposed approach . . . . . . . . . . . . . . . . 56

4.6 Field survey . . . . . . . . . . . . . . . . . . . . . . . . . . . . 58

4.7 Field data processing and analysis . . . . . . . . . . . . . 62

4.7.1 Data quality and uncertainty . . . . . . . . . . . 62

4.7.2 Homogeneous halfspace model . . . . . . . . . . . . . . 63

4.7.3 Layered Earth model . . . . . . . . . . . . . . . . . . . . 65

4.8 Summary and conclusions . . . . . . . . . . . . . . . . 72

4.9 Acknowledgments . . . . . . . . . . . . . . . . . . . . . . . . . . . . 73

5 Coupling of VLF TTER signals to elongated conductors $\quad 74$

5.1 Abstract . . . . . . . . . . . . . . . . . . 74

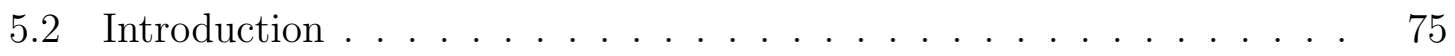

5.3 Theoretical background . . . . . . . . . . . . . . . . 77

5.4 Methods . . . . . . . . . . . . . . . . . . . 78

5.4 .1 TTE radio system $\ldots \ldots \ldots \ldots \ldots \ldots$

5.4.2 Finite-difference time-domain modelling . . . . . . . . . 79

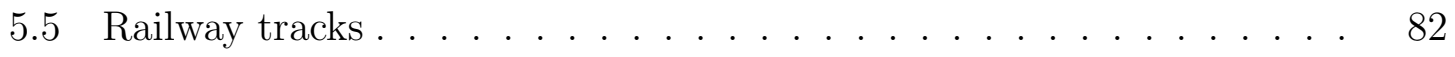

5.5 .1 Underground railway tracks . . . . . . . . . . . . . . 82

5.5 .2 Surface railway tracks f . . . . . . . . . . . . . . 82

5.5 .3 Numerical modelling . . . . . . . . . . . . . . . 85

5.6 Elevator shaft $\ldots \ldots \ldots \ldots \ldots \ldots \ldots \ldots$

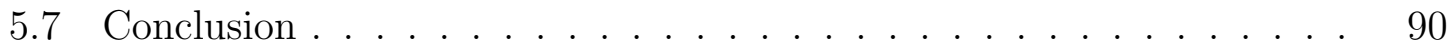

5.8 Acknowledgments . . . . . . . . . . . . . . . . . . . . . . . . 92

6 Near-field VLF EM signal propagation in multistory buildings 94

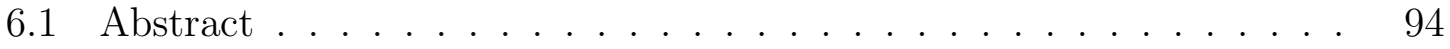

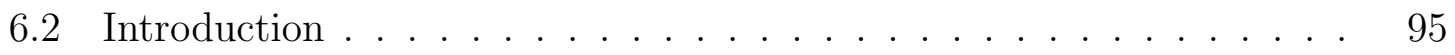


6.3 Methodology . . . . . . . . . . . . . . . . 97

6.3.1 Through-the-Earth radio system . . . . . . . . . . . . . 97

6.3.2 Finite-difference time-domain modelling . . . . . . . . . . 97

6.3.3 Thin sheets . . . . . . . . . . . . . . . . . 98

6.3.4 Synthetic data . . . . . . . . . . . . . . . . . . 99

6.3.5 Conductance model . . . . . . . . . . . . . . . . 100

6.4 Case studies . . . . . . . . . . . . . . . . . . . . . . . 101

6.4.1 Parking garage . . . . . . . . . . . . . . . 101

6.4.2 Hotel . . . . . . . . . . . . . . . . . . . 104

6.4.3 Embassy . . . . . . . . . . . . . . . . . . . . 108

6.4.4 Loeb Building . . . . . . . . . . . . . . . . . . 110

6.5 Discussion . . . . . . . . . . . . . . . . . . . . . . . . . . 112

6.6 Conclusion . . . . . . . . . . . . . . . . . . . . . 115

6.7 Acknowledgments . . . . . . . . . . . . . . . . 116

$\begin{array}{lll}7 & \text { Conclusion } & 117\end{array}$

7.1 Summary . . . . . . . . . . . . . . . . . 117

7.2 Future work . . . . . . . . . . . . . . . . . 119

$\begin{array}{ll}\text { List of References } & 120\end{array}$ 


\section{List of Tables}

4.1 Deviation of observed and calculated $Q$ in decibels for the model shown in Figure $4.11 \ldots \ldots \ldots \ldots$

5.1 CanaryTalk TTE radio system specifications . . . . . . . . . . 79

6.1 Conductance model specifications and comparison of signal strength . 100 


\section{List of Figures}

1.1 Examples of TTE radio applications $\ldots \ldots \ldots \ldots \ldots$

3.1 Benchmarking of GPU-accelerated FDTD code $\ldots \ldots \ldots \ldots$

3.2 Configuration for a square loop $\ldots \ldots \ldots \ldots \ldots$

3.3 Cosine-modulated Gaussian function . . . . . . . . . . . 30

3.4 Vertical magnetic field in free air . . . . . . . . . . . . . . 31

3.5 Vertical magnetic field in homogeneous halfspace . . . . . . . . . . 32

3.6 Vertical magnetic field in three-layer Earth . . . . . . . . . . . 34

3.7 Total magnetic field in faulted coal-bearing stratigraphy $\ldots \ldots \ldots$

3.8 Time-dependent magnetic field in faulted coal-bearing stratigraphy . 37

3.9 Total magnetic field in salt-bearing stratigraphy $\ldots \ldots \ldots \ldots$

3.10 Time-dependent magnetic field in salt-bearing stratigraphy . . . . . . 40

4.1 Vertical magnetic field in a homogeneous halfspace versus conductivity 55

4.2 Vertical magnetic field in a homogeneous halfspace versus frequency . 55

4.3 Vertical magnetic field in a homogeneous halfspace versus radial distance 56

4.4 Fourier spectra of synthetic data generated using the FDTD method . 58

4.5 Map and 3D view of Safety Research Coal Mine . . . . . . . . . . 59

4.6 TTE radio interharmonic modulation (IHM) waveform in time domain and frequency domain. . . . . . . . . . . . . . 61 
4.7 Propagation factor $Q$ versus frequency for receiver 9 and transmitter

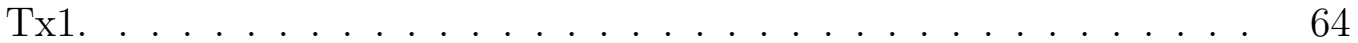

4.8 Propagation factor $Q$ at location 9 and transmitter Tx1 for four representative frequencies. . . . . . . . . . . . . . . . . . 64

4.9 Apparent conductivity versus frequency for receiver 9 and transmitter

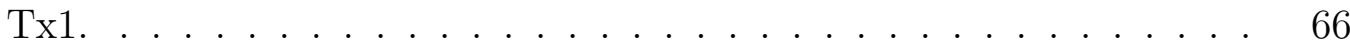

4.10 Flowchart for fitting a four-layer conductivity model . . . . . . . . . . 67

4.11 Four-layer model with fitted conductivities for each layer. . . . . . . . 70

4.12 Calculated and observed data after fitting a conductivity model to the

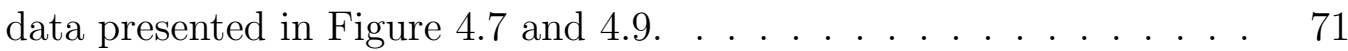

5.1 Excitation of a horizontal conductor by a vertical magnetic dipole. . . 77

5.2 Comparison between induced axial current calculated analytically and with FDTD . . . . . . . . . . . . . . . . . . . . 81

5.3 Equipment setup along surface railway tracks at Chelsea, Canada . . 84

5.4 Signal decay along surface railway tracks at Chelsea, Canada. . . . . 84

5.5 Signal strength and rate of change with distance along railway tracks 86

5.6 Architectural layout of Dunton Tower . . . . . . . . . . . . . . . . . . 88

5.7 Comparison of signal strength in Dunton Tower measured in the field and computed using FDTD. . . . . . . . . . . . . . . . . . . . . 90

5.8 Signal strength in Dunton Tower matched to field data for small loop. $\quad 91$

5.9 Signal strength, baseline with no elevators, in Dunton Tower using small loop. . . . . . . . . . . . . . . . . . . . . . . . . . . 91

6.1 Model of parking garage . . . . . . . . . . . . . . . . . . . . . 102

6.2 Comparison between observed and modelled data in parking garage . 103

6.3 Modelled signal strength in the parking garage . . . . . . . . . . 105

6.4 Model of hotel . . . . . . . . . . . . . . . . . . . 106 
6.5 Comparison between observed and modelled data in hotel . . . . . . . 107

6.6 Model of embassy . . . . . . . . . . . . . . . . . . . . . . . . . 108

6.7 Comparison between observed and modelled data in embassy . . . . . 109

6.8 Model of Loeb Building . . . . . . . . . . . . . . . . . . . . . 110

6.9 Comparison between observed and modelled data in Loeb Building 111

6.10 Modelled signal strength in Loeb Building . . . . . . . . . . . . . . . 112 


\section{Chapter 1}

\section{Introduction}

\subsection{Through-the-Earth radio}

Through-the-Earth (TTE) radio has been proposed as a means of wireless communications, often for emergencies, in a variety of settings that are difficult to access or are entirely inaccessible by conventional means. Several applications have been proposed for TTE radio (Figure 1.1). A particularly important setting is underground environments, such as mines, civilian infrastructure such as tunnels for utilities or transportation, or parking garages. Other examples include multistory buildings, especially those that are difficult to access by conventional means, military applications, and even wireless detonation. The distinguishing feature of TTE radio is that its operating frequency is in or below the VLF band (very low frequency; $3-30 \mathrm{kHz}$ ), which permits wireless communications in most geological settings. The TTE radio systems used in this study have a range of 400-9000 Hz, which corresponds to the VLF and ULF (ultra low frequency; 300-3000 Hz) bands. In underground mines in particular, other communication tools exist, such as line-of-sight systems, wired communications, and leaky feeder systems, which are conceptually a combination of a wired and a line-of-sight system where a signal will "leak" from an uninsulated wire 


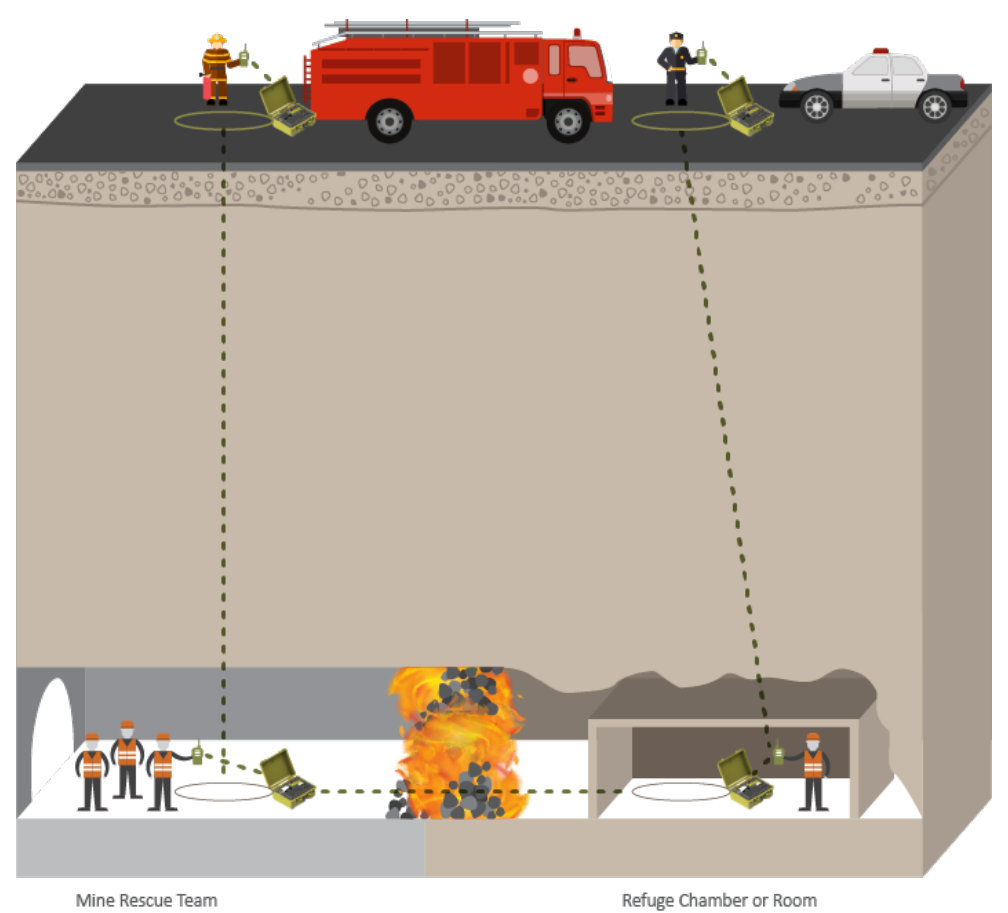

(a)

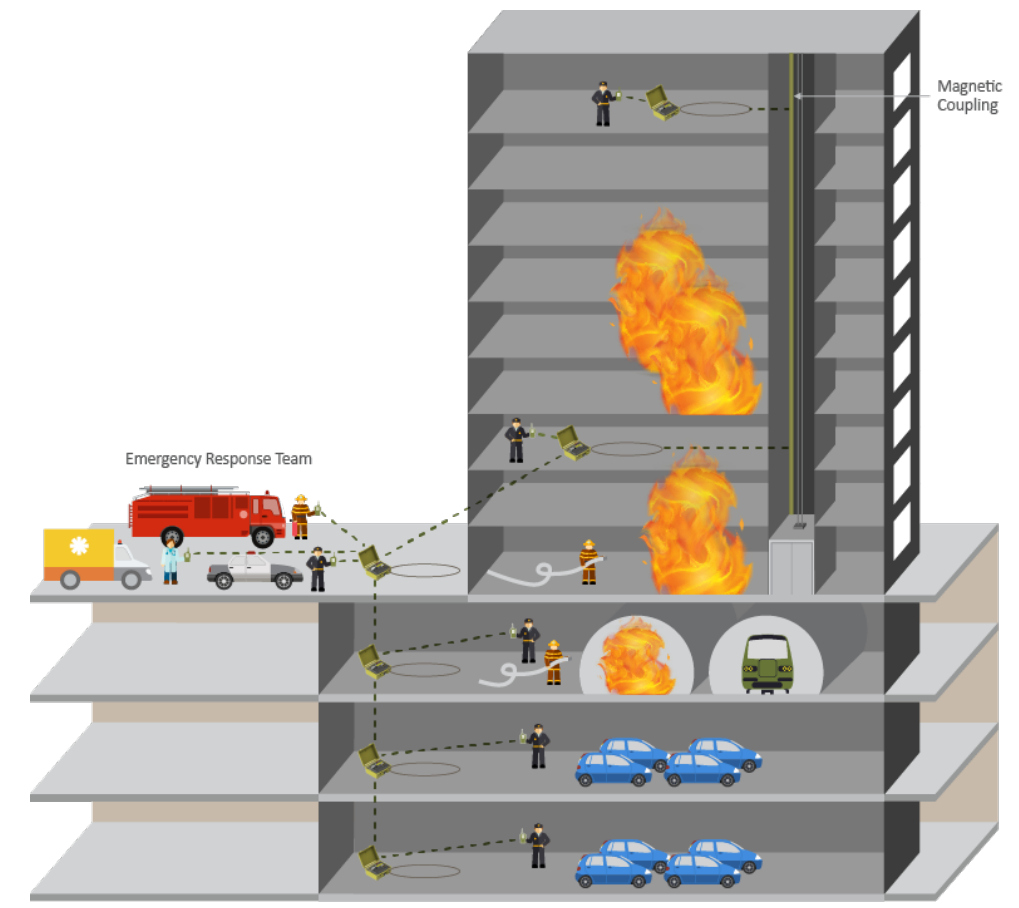

(b)

Figure 1.1: Examples of TTE radio applications in underground mines (a) and in multistory buildings (b). Reproduced with permission of Vital Alert Communication, Inc. 
(Delogne, 1982; Forooshani et al., 2013; Yarkan et al., 2009). The major advantage of TTE systems over these systems is that it can remain operational even in the case of incidents such as mine collapses, rock bursts, explosions, floods, or fires. The majority of TTE systems operate on magnetic induction, where an alternating current is run in a transmitting loop, which, per Faraday's law, induces a time-varying magnetic field, which in turn induces a voltage across either another wire loop or a ferrite coil, which acts as the receiver. In contrast, there also exist electric field or galvanic TTE radio systems, which operate on sensing currents injected into the ground by a pair of electrodes. The drawback of such systems is higher noise (Yenchek et al., 2012). A further limitation in TTE communications, regardless of whether it is an inductive or galvanic system, is that signal strength cannot be arbitrarily increased, for two reasons: first, it is a common requirement that systems remain portable, and second, to reduce the risk of a spark if operating in a methane-air mixture, which can be the case after a coal mine explosion.

TTE radio by itself is not a novel technology. Nikola Tesla proposed wireless transmissions through the Earth as early as 1899 (Forooshani et al., 2013; Yarkan et al., 2009), and Guglielmo Marconi was believed to have understood the limitations the ground posed to conventional radio (Wait, 1975). In World War I, grounded electrodes were used to communicate between trenches; this method is referred to as télégraphie par sol (TPS), or telegraphy through the ground (Williams, 1951). Considerable further research was done on the feasibility of this technology during the 1920s (Ilsley et al., 1928; Felegy and Coggeshall, 1948; Large et al., 1973) by the Bureau of Mines in the United States, and during the mid-1930s in Nazi Germany (Felegy and Coggeshall, 1948). This research was largely abandoned by World War II (Durkin, 1982; Hill and Wait, 1982), at least as evidenced by the paucity of contributions to the scientific literature, although further military research is entirely possible 
given the applications of such technology. Work largely focused on evaluating the feasibility of TTE communications was done after the war by both the governmental Bureau of Mines (Coggeshall et al., 1948; Felegy and Coggeshall, 1948) and by a private military contractor (Ames et al., 1963).

Further work was undertaken on TTE radio in the mid 1970s to early 1980s, with less of a military orientation (Delogne, 1982). In contrast to the previous theme of assessing the feasiblity of a communication link, more varied studies were done. One avenue of research was direction finding, where it was investigated whether below ground signals could be used to estimate the position of a trapped miner (Geyer and Keller, 1976; Hill and Wait, 1982; Lagace et al., 1980; Large et al., 1973; Olsen and Farstad, 1973; Pittman et al., 1985), although this problem largely remains open today (Sogade et al., 2004; Ayuso et al., 2010; Sheinker et al., 2014) given the complexity involved in accounting for the distortion of an electromagnetic signal by a conductive ground. Another line of work was using TTE transmissions to constrain the conductivity structure of a mine overburden (Geyer et al., 1974; Lagace et al., 1980; Durkin, 1984a, 1991), by measuring the received signal underground. It builds upon work done after World War II on the transmission of signals usually of conventional radio frequencies through highly resistive overburdens (Haycock et al., 1949; Barret, 1952; Pritchett, 1952; McGehee, 1954; Pullen, 1953; Dokoupil et al., 1962; Fritsch, 1963). Theoretical work has been done in the context of TTE communications, where analytic solutions for the propagation of electromagnetic energy through relevant media, such as a layered Earth, have been derived. The work of J. R. Wait is noted in particular, who considered layered Earth situations, propagation in coal seams, waveguide effects, and wired systems (Hill and Wait, 1974; Tripp et al., 2000; Wait, 1954, 1970, 1971, 1975, 1982, 1986; Wait and Spies, 1972).

Recent work on TTE radio has been motivated by the passage of the United States 
Mine Improvement and New Emergency Response (MINER) act (June 2006), which prescribes a requirement to have wireless communications between the mine level and the surface in underground mines to improve the likelihood of rescue. Further work has been done for modelling TTE radio transmission (Gibson, 2003; Yan et al., 2013; Yan, 2014), which has also included revisiting the data obtained in the 1970s for determining mine overburden conductivity based on TTE radio transmissions (Yan et al., 2014). Another line of research deals with a phenomenon, observed in testing of VLF TTE radios, where there is a marked increase in signal range in the presence of elongated conductors. This effects is also noted in the MF band (medium frequency; 300-3000 kHz). In this band, the effect has been observed since the 1920s (Carson, 1926; Stolarczyk, 1991). Recent work has treated the phenomenon in the MF range as a transmission line effect (Li et al., 2012, 2013, 2014; Brocker et al., 2015). This effected in the MF band is exploited in geophysical detection of such objects (McKenna and McKenna, 2010; McKenna et al., 2013; Naus, 2013); in contrast, most electromagnetic exploration geophysical systems use frequencies in the VLF band and below to penetrate the ground to a greater depths.

\section{$1.2 \quad$ Statement of problem}

Previous work, both of a practical and theoretical nature, has shown unambiguously that TTE radio is a viable technology. Existing work on modelling the propagation of a TTE radio signal is nevertheless lacking. Information derived from modelling can be critical in numerous situations, such as to optimize transmitter and receiver placement, in determining if a TTE link is at all possible in a given setting, or what is the maximum transmitter-receiver spacing at which a link remains possible. Prior work has been either experimental in nature, which involved testing the hardware 
required for a fully functional TTE radio system, or was based on analytical equations to predict signal strength at depth. The complex geometry of complicated geological settings, in the presence of elongated conductors, or even in a non-geologic setting such as a multistory building, cannot be adequately described by analytical solutions. Numerical modelling was done in Yan et al. (2013) to verify analytic results but was not extended to an arbitrary geometry. The absence of sophisticated modelling is in part attributable to a previous lack of computing power to efficiently handle highly inhomogeneous media. Additionally, TTE transmissions have not been systematically studied in the presence of elongated conductors or in multistory buildings, while such applications of the technology have been proposed. Indeed, the propagation of VLF waves in multistory buildings has only been investigated for the penetration of far-field VLF signals from military transmitters (Smith, 1978).

A problem related to producing sophisticated models of TTE radio signal propagation is to determine the conductivity distribution of a transmission setting, which is the most important physical property that controls the propagation of the signal. Any model is only as good as the conductivity model underlying the simulation. Previous work has used TTE radio signals to estimate the subsurface conductivity distribution, but the methodology was largely based on fitting a regression model to US coal mines (Durkin, 1984a). This approach further used one transmitter-receiver orientation with only a few frequencies and idealized models of the subsurface (e.g. homogeneous halfspace). Site-specific information would yield a better result, and in the case of propagation through more complex media, such as in the presence of elongated conductors or in multistory buildings, simple models such as homogeneous halfspaces can no longer be used, and appropriate conductivities must be supplied for the model to be representative. 


\subsection{Objectives and originality}

The objectives of this research are broadly split among four avenues of investigation, which correspond each to a paper included in the integrated thesis:

1. To develop a robust and efficient forward modelling tool optimized for simulating VLF signals in a variety of complex media

2. To estimate a site-specific conductivity structure by measuring a transmitted VLF TTE radio signal at several locations and frequencies

3. To characterize, experimentally and numerically, the coupling of a VLF signal to elongated conductor and apply it to TTE radio

4. To characterize, experimentally and numerically, the propagation of a VLF signal in multistory buildings and apply it to TTE radio

All VLF signals referenced are near-field inductive TTE radio signals, but the work is applicable to any near-field signal of a similar frequency. These objectives build upon and extend existing knowledge about TTE radio, and form together a coherent body of work. The first objective improves on existing methods of forward modelling of TTE radio, which furthers the knowledge of how a VLF signal propagates in inhomogeneous conductive media. The second objective builds on previous general studies (e.g. Durkin (1984a)) by considering site-specific information, and several transmitter-receiver orientations and frequencies; both approaches are more geophysically robust as even for US coal mines, there is considerable variation in parameters that would influence the conductivity distribution, such as geological structure. This second objective builds on the first objective, as the forward modeller is used for data analysis. The major contribution to knowledge in the third and fourth objectives is 
that the propagation of near-field VLF signals is systematically studied in two situations previously not considered in the literature, namely in the presence of elongated conductors and in multistory buildings. Furthermore, these last two objectives build on the first two objectives, as they rely on having a robust and efficient forward modeller optimized for VLF signals, and the ability to estimate a site-specific conductivity distribution. Combined together, these objectives address the existing scientific and engineering problem of the inability to estimate the performance or viability of a TTE radio system at a given site for lack of an adequate understanding of how the TTE radio signal will propagate at the given site, by developing a tool to efficiently model such sites and by developing a methodology to estimate the site-specific conductivity structure that controls signal propagation. 


\section{Chapter 2}

\section{Methodology}

\section{$2.1 \quad$ Overview}

This thesis follows a common methodology involving the use of a finite-difference time-domain (FDTD) code to model through-the-Earth (TTE) radio transmissions, and making measurements with the TTE radio receivers or a noise measurement unit termed ANDAU (Automated Noise Data Acquisition Unit) to acquire data to validate models or to use to fit conductivity models. The four body chapters in this thesis each represent a standalone manuscript published, accepted, or under review in a peer-reviewed journal (see Preface). As such, they are written to fully describe the methodological approach used in each case. Chapter 3 describes only the validation of the FDTD code with analytical methods as applied to TTE radio communications, whereas Chapters 4-6 integrate both field measurements and modelling results. A concise summary of the common methodological considerations common to the thesis is described as follows. 


\subsection{Finite-difference time-domain method}

Finite-difference time-domain (FDTD) is a numerical technique used to solve Maxwell's equations, which describe all classical electromagnetic phenomena. An original code implementing FDTD was developed for use in this research. First, it was used to model the TTE radio transmissions; the system in question uses magnetic induction in a frequency range from 400 to $9000 \mathrm{~Hz}$ to wirelessly transmit signal through a conductive overburden. The numerical results were validated based on comparison to analytical methods and field data. Second, the FDTD code was used to fit conductivity models to field data, which was applied to mine overburdens, coupling of signal to elongated conductors, and in multistory buildings. A detailed discussion of the theoretical basis of the FDTD method is beyond the scope of the thesis, and the reader is referred to Taflove and Hagness (2005) or Elsherbeni and Demir (2009). Instead, a brief background and details on the implementation are provided. These details are largely shared between all body chapters.

FDTD is a differential equation method, and starts from the curl form:

$$
\nabla \times \mathbf{E}=-\mu \frac{\partial \mathbf{H}}{\partial t}
$$

and

$$
\nabla \times \mathbf{H}=\sigma \mathbf{E}+\epsilon \frac{\partial \mathbf{E}}{\partial t},
$$

where $\mathbf{E}$ is the electric field; $\mathbf{H}$ is the magnetic field; $\sigma$ is the conductivity of the medium; $\mu$ is the permeability of the medium; and $\epsilon$ is the permittivity of the medium.

To discretize the electric and magnetic fields, the Yee cell (Yee, 1966) is used. In this configuration, the $\mathbf{E}$ and $\mathbf{H}$ fields are offset both spatially and temporally by a half cell and time step, respectively, which permits an explicit solution. The geometrical 
domain is discretized in rectangular cells, which is a simpler approach, both in terms of algorithm complexity and computational resources, compared to a finite-element scheme. Each cell is assigned a value for the three electromagnetic properties of interest, namely $\sigma, \mu$, and $\epsilon$. The electromagnetic property of greatest importance for TTE radio is the conductivity $\sigma$, which varies logarithmically in geologic and manmade materials. In contrast, the magnetic permeability $\mu$ is largely constant outside of magnetic materials (e.g. magnetite), whereas the effect of the electric permittivity $\epsilon$ is largely insignificant at the very low frequencies used in this application. When energy reaches the edge of the grid, numerical reflections occur. To mitigate these reflections, the convolutional perfectly matched layer (CPML) absorbing boundary condition $(\mathrm{ABC})$ is used. CPML cells are added to the edges of the computational domain to absorb incoming electromagnetic energy - the mechanical analogy is an anechoic chamber. Unlike, for example, a perfect electric conductor ABC, perfectly matched layer (PML) ABCs are designed to not reflect energy back into the grid.

The time step used is a function of the cell size. Assuming a cubic cell with side length $\Delta s$, the maximum time step $\Delta t$, as given by the Courant-Friedrich-Levy stability criterion, is

$$
\Delta t=\frac{\Delta s}{v_{c} \sqrt{3}},
$$

where $v_{c}$ is the speed of light in the medium of interest. If a bigger time step is used, the FDTD algorithm will diverge. This limitation presents a challenge for modelling signals in a frequency range of 400-9000 Hz. First, modelling a geometrical domain on order of $100 \mathrm{~m}$ across at a resolution of $1 \mathrm{~m}$ would require millions of cells, which results in a considerable requirement for both computational memory and execution time. 
To improve performance, the FDTD code is parallelized and executed on a generalpurpose graphics processing unit (GPU). In comparison to a single-thread Fortran implementation of the code, a cubic domain with a side length of 50 cells can be processed approximately 24 times faster, i.e. what would initially require a day to compute can be done in an hour. This comparison is not entirely representative of usual operating conditions, as a bigger geometrical domain is used. The computational throughput (how many cells are processed per second) increases as more of the GPU memory becomes saturated, and for a domain with a side length of 300 cells, is tenfold compared to that for 50 cell cube. FDTD is particularly well suited for parallelization. Due to the leapfrog nature of the algorithm, where the magnetic field is dependent on the electric field in the previous time steps, all cells for all three components of either field can be updated simultaneously. While there exist unconditionally stable variations of the FDTD method, they are more difficult to parallelize and suffer from accuracy problems when the chosen time step is too large.

\subsection{Through-the-Earth radio equipment}

In this study, the Canary series of TTE radios made by the industrial sponsor of this research, Vital Alert Communication Inc., were used. During the course of this research, several models of Canary TTE radios were used, as a response to improvements to their design. The transmitted magnetic field, which uses either standard quadrature phase-shift keying (QPSK) modulation or Canary-specific interharmonic modulation (IHM) (cf. Chapter 4), does not fundamentally change.

A TTE radio consists of a main terminal, to which a transmitter and receiver is connected. The main terminal is a Pelican case which houses the power supply and hardware such as the circuit boards. The transmitter is a loop of wire that 
is connected to the terminal. The receiver, originally also a loop of wire, is now a box with three mutually orthogonal ferrite coils (also termed 3D receiver). Each terminal has both a transmitter and receiver to permit two-way communications with another terminal. Two-way communications were not the focus of research; instead, one terminal was designated as the transmitting unit whereas the second terminal, the receiver unit, was used to measure the signal strength, so that it could be used to derive information about the conductivity structure of a transmitting environment, or to validate the FDTD model.

In Chapter 4, it is demonstrated that a TTE radio signal can be used to estimate the conductivity structure of a mine overburden. While the same transmitter set up was used as in the other experiments, in this case we used Vital Alert's Automated Noise Data Acquisition Unit (ANDAU) to record the transmitted signal at depth, instead of using the TTE radio receiver. While the ANDAU was originally designed to measure a time series of ambient noise conditions, it can equally be used to record the time series of the transmitted signal at depth. By taking a Fourier transform of this time series, the signal strength at multiple frequencies can be determined, as opposed to the signal strength corresponding to the central frequency of the TTE radio signal as measured with the receiver that is attached to the terminal. 


\section{Chapter 3}

\section{Finite-difference time-domain modelling of through-the-Earth radio signal propagation}

\subsection{Abstract}

This research seeks to extend the knowledge of how a very low frequency (VLF) through-the-Earth (TTE) radio signal behaves as it propagates underground, by calculating and visualizing the strength of the electric and magnetic fields for an arbitrary geology through numeric modelling. To achieve this objective, a new software tool has been developed using the finite-difference time-domain method. This technique is particularly well suited to visualizing the distribution of electromagnetic fields in an arbitrary geology. The frequency range of TTE radio (400-9000 Hz) and geometrical scales involved ( $1 \mathrm{~m}$ resolution for domains a few hundred meters in size) involves processing a grid composed of millions of cells for thousands of time steps, which is computationally expensive. Graphics processing unit acceleration was used to reduce execution time from days and weeks, to minutes and hours. Results from the new modelling tool were compared to three cases for which an analytic solution 
is known. Two more case studies were done featuring complex geologic environments relevant to TTE communications, that cannot be solved analytically. There was good

agreement between numeric and analytic results. Deviations were likely caused by numeric artifacts from the model boundaries; however, in a TTE application in field conditions, the uncertainty in the conductivity of the various geologic formations will greatly outweigh these small numeric errors.

\subsection{Introduction}

Through-the-Earth (TTE) radio has been proposed as a means of emergency communication in a variety of subterranean situations, such as in mining and transportation tunnels. A typical TTE radio communication system includes a node located at the surface and a node placed underground. To enable two-way communication, both nodes are equipped with a transmitter $(\mathrm{Tx})$ and receiver $(\mathrm{Rx})$. TTE radio works through electromagnetic (EM) induction in the extremely low to very low frequency (ELF-VLF) band - in practice, between 400-9000 Hz, in order to reach depths down to $500 \mathrm{~m}$ in mining applications. Two-way text and voice data can be transmitted. Voice transmission requires higher frequencies, as a sufficiently high bit rate is needed, which comes at the expense of signal range, as higher frequencies get attenuated more in materials. The TTE link provides a means of communication in an emergency where the conventional wire-based systems have been compromised by fire, explosion, or flooding.

It is well known that electromagnetic signals, even at low frequencies, will get attenuated as they propagate through the conductive Earth. Some basic analytic solutions are available to calculate signal strength along the transmission path; however, these apply to simple one-dimensional cases and often reduce the transmitter to 
an idealized dipole. Displacement currents that are relevant to more resistive geologic bodies are ignored, and many other critical factors, such as external noise, geologic complexity, and infrastructure (cultural effects), cannot be accounted for. Furthermore, solutions are typically in the frequency domain and are thus applicable for a single frequency of interest, which is not the ideal approach for handling a modulating TTE signal, or to account for various sources.

This research seeks to extend the knowledge of how VLF signal behaves as it propagates through the Earth by calculating and visualizing the strength of the electric and magnetic fields for an arbitrary geology through numeric modelling. A new modelling tool will be validated by performing simulations for three cases for which an analytic solution exists. Then, two cases featuring complex geologic environments for which an analytic solution does not exist will be presented. In particular, a faulted coal seam will be modelled. TTE communication has particular application in coal mines, which are especially prone to accidents, explosions, or collapses that can sever any through-the-air (TTA) or through-the-wire (TTW) communication links. As such, it is important to understand how the magnetic field propagates in such environments so that a TTE system could be strategically installed.

\subsection{Literature review}

The first proposal for the transmission of a signal through the Earth was made as early as 1899 by Nikola Tesla (Forooshani et al., 2013; Yarkan et al., 2009), although Guglielmo Marconi arguably understood the limitations to conventional radio communications posed by the ground (Wait, 1975). Wireless communication between trenches through grounded electrodes was accomplished during World War I. Experiments were also done with induction-based systems for potential military applications, 
during World War I (Williams, 1951) and the 1920s (Large et al., 1973), but encouraging results were not obtained, and the technology was largely abandoned by World War II (Durkin, 1982; Forooshani et al., 2013; Hill and Wait, 1982). More work on TTE communications was undertaken in the mid 1970s to early 1980s, this time with civilian applications in mind as opposed to previous military-oriented research (Delogne, 1982). Of particular interest was direction finding (Geyer and Keller, 1976; Hill and Wait, 1982; Lagace et al., 1980; Large et al., 1973; Olsen and Farstad, 1973; Pittman et al., 1985), where an operator on the surface attempts to locate a trapped miner. For example, one could measure the horizontal magnetic field from an underground horizontal loop. Assuming the transmitter is small enough to be worn, a miner could be located by finding the null of the field, which occurs in the centre of the loop. Considerable work was also done on rock properties (Durkin, 1982), as the critical effect overburden conductivity has on the ability to transmit and receive communications was widely recognized by that time (Yan et al., 2013). A recent law, the United States Mine Improvement and New Emergency Response (MINER) act (June 2006), has stimulated further research in TTE communications (Forooshani et al., 2013; Yenchek et al., 2012). The MINER act aims to increase the likelihood of rescue by mandating wireless communications between trapped miners and the surface (Yan et al., 2013).

The advantage of a TTE communication link is that it can remain operational even in the case of incidents such as mine collapse, rock burst, explosion, flood, or fire. In contrast, TTA, where the signal passes along a line of sight, TTW, and leaky feeders, which is a permutation of TTA and TTW where signal "leaks" from an uninsulated wire (Delogne, 1982), are all vulnerable to destruction in the aforementioned incidents (Forooshani et al., 2013; Yarkan et al., 2009). Nevertheless, there are major difficulties with TTE radio communication. There is significant noise in the typical operating 
frequency range (400-9000 Hz), which includes lightning strikes (sferics), ionospheric interactions, and cultural noise, especially AC (50 or $60 \mathrm{~Hz}$ ) harmonics (Okada and Iwai, 1988; Watt, 1967). Electromagnetic induction, as opposed to grounded dipoles, is typically used for TTE radio, as the background noise is lower in the magnetic channels as opposed to the electric channels. Nevertheless, fighting noise simply by increasing signal strength is not option, as it would require too much power, which is dangerous in a potentially methane-rich atmosphere (Yenchek et al., 2012).

Previous modelling work has largely been analytic. The propagation of an electromagnetic wave through a layered medium has been considered in some detail in the literature, although these analytic solutions are limited to simple geometries and excitations. Of particular importance is the work of J.R. Wait, which includes extensive publications on layered Earth scenarios, propagation in coal seams, waveguide effects, cable-based communications, ambient mine field intensity, and TTE communications (Hill and Wait, 1974; Tripp et al., 2000; Wait, 1954, 1970, 1971, 1975, 1982, 1986; Wait and Spies, 1972).

In this paper, three analytic cases are considered. First, a simple Biot-Savart law example, per Griffiths (1999), is demonstrated; next, the results for a homogeneous halfspace are compared to those of Wait and Spies (1972) (cf. Appendix 3.8); and finally, a three-layer Earth, per Shope (1982), which builds upon Wait and Spies (1972), is modelled (cf. Appendix 3.9). A homogeneous halfspace was previously successfully numerically modelled by Yan et al. (2013) using a method-of-moments solver, and compared to the results from Wait and Spies (1972). However, more realistic numeric models have not been previously attempted. The new modelling tool has full 3D capabilities, which constitutes the main contribution of this paper. 


\subsection{Methods}

\subsubsection{Finite-difference time-domain method}

The finite-difference time-domain (FDTD) method, first proposed by Yee (1966), is a fully explicit solution to Maxwell's curl equations. As a time-domain solution, it is capable of modelling arbitrary excitations and generating a time series of the electromagnetic behavior. It uses no linear algebra, and is easily parallelizable(Simpson, 2012).

As a differential equation method, FDTD begins with Maxwell's curl equations (Elsherbeni and Demir, 2009; Griffiths, 1999; Taflove and Hagness, 2005):

$$
\nabla \times \mathbf{E}=-\mu \frac{\partial \mathbf{H}}{\partial t}
$$

and

$$
\nabla \times \mathbf{H}=\sigma \mathbf{E}+\epsilon \frac{\partial \mathbf{E}}{\partial t},
$$

where $\mathbf{E}$ is the electric field; $\mathbf{H}$ is the magnetic field; $\sigma$ is the conductivity of the medium; $\mu$ is the permeability of the medium; and $\epsilon$ is the permittivity of the medium.

The parameters $\sigma, \mu$, and $\epsilon$ are the fundamental electromagnetic properties. The conductivity $\sigma$ varies over several orders of magnitude for geologic materials; the permeability $\mu$ can be assumed constant outside of magnetic materials (e.g. magnetite, pyrrhotite); and the permittivity $\epsilon$ can be assumed largely irrelevant at the frequencies used in TTE communications (cf. Section 3.4.2).

The curl equations are discretized and rearranged to form recursive update equations, with constants based on the material properties $\sigma, \mu$, and $\epsilon$, a time step $\Delta t$, and a grid with spacings of $\Delta x, \Delta y$, and $\Delta z$ : 


$$
\begin{aligned}
E_{x}^{n+1}(i, j, k)= & C_{\text {exe }}(i, j, k) \times E_{x}^{n}(i, j, k)+ \\
& C_{\text {exhz }}(i, j, k) \times\left(H_{z}^{n+\frac{1}{2}}(i, j, k)-H_{z}^{n+\frac{1}{2}}(i, j-1, k)\right)+ \\
& C_{e x h y}(i, j, k) \times\left(H_{y}^{n+\frac{1}{2}}(i, j, k)-H_{y}^{n+\frac{1}{2}}(i, j, k-1)\right)+ \\
& C_{e x j}(i, j, k) \times J_{i x}^{n+\frac{1}{2}}(i, j, k) .
\end{aligned}
$$

The reader is directed to Taflove and Hagness (2005) or Elsherbeni and Demir (2009) for the complete FDTD updating equations.

A major challenge in FDTD is grid termination. When energy propagates through discrete space, it reaches the end of the grid, which presents a discontinuity that causes numeric reflections, and impacts the results. To reduce this numeric error, energy must be absorbed at the edges; the mechanical analogy is an anechoic chamber. The absorption is achieved using the convolutional perfectly matched layer (CPML) (Roden and Gedney, 2000). Perfectly matched layers are a fictitious material added to the grid boundaries; they are reflectionless regardless of the angle of incidence. Convolution was added after the original PML scheme was postulated (Elsherbeni and Demir, 2009; Taflove and Hagness, 2005) to resolve late-stage instability and to better handle different materials.

FDTD is mostly used in radio frequency $(\mathrm{RF})$ engineering, photonics, radar, and microwave applications; it has been applied to geophysics previously, but not specifically to TTE radio communication. Examples of geophysical applications include modelling the Earth-ionosphere waveguide (Simpson, 2012; Taflove and Hagness, 2005); modelling the transient EM response of the Earth (Wang and Hohmann, 1993); forward modelling and inversion of magnetotelluric data to delineate a salt dome for 
petroleum exploration (de la Kethulle de Ryhove et al., 2013; de la Kethulle de Ryhove and Mittet, 2014); dispersion in ground-penetrating radar surveying (Bergmann et al., 1998); and controlled-source electromagnetic (CSEM) seafloor exploration for petroleum exploration (Dukeshire et al., 2011; Niu and Simpson, 2012).

In geophysics, numeric methods other than FDTD have been used, especially integral equation (IE) and finite-element (FE) methods. The integral equation methods are often avoided, as the computation of the required system matrix is an extremely complicated problem by itself, before even solving for field values (Adveev, 2005). A related technique, the method-of-moments has been used to model a simplistic TTE scenario (Yan et al., 2013), but it is poorly suited to handle inhomogeneous media and dielectrics. The finite-element method is primarily a frequency domain method with limited time-domain variants (Lee et al., 1997). This technique fits a tetrahedral mesh to the computational domain, and unlike finite-difference methods which fit a grid, usually rectangular, it can effectively model non-rectangular geometries, or features at disparate scales. The drawback is that mesh generation remains a major open question, in addition to the speed and robustness of the approach (Adveev, 2005; Lee et al., 1997). While FE methods arguably generate better meshes than finitedifference methods, the added complication and the nature of the geometry model, along with the difficulty in generating a finite-element mesh, do not justify using them. Furthermore, the linear algebra inherent to the IE and FE methods presents a computational bottleneck (Simpson, 2012). As such, FDTD is the method of choice for modelling TTE communications. It handles the requisite materials better; it can be parallelized easily; it requires no linear algebra; and it is a time-domain technique that shows the propagation of the signal over time in a straightforward manner. Although more established, frequency-domain techniques handle one frequency at a time and are best suited for periodic signals. Using a time-domain technique simplifies the 
modelling of transient signals, which gives the flexibility of modelling various types of possible TTE signals, which is useful for further research and development of the technology. In this study, a broadband excitation is used to approximate a typical TTE signal (cf. Section 3.5.1). Furthermore, a time-domain modeler allows the superposition of various signals. Of particular interest are lightning strikes (sferics) and cultural noise (e.g. from $50 \mathrm{~Hz}$ or $60 \mathrm{~Hz}$ AC power lines) - in the frequency domain, modeling simultaneously this variety of sources is more difficult.

\subsubsection{Optimization}

A drawback of the FDTD method is its long execution time. This numeric method is only conditionally stable; the Courant-Friedrichs-Levy (CFL) stability criterion prescribes the longest possible time step:

$$
\Delta t \leq \frac{1}{v_{c} \sqrt{\frac{1}{(\Delta x)^{2}}+\frac{1}{(\Delta y)^{2}}+\frac{1}{(\Delta z)^{2}}}}
$$

where $v_{c}$ is the speed of light in a given medium (Elsherbeni and Demir, 2009; Taflove and Hagness, 2005). For a uniform grid (i.e. $\Delta x=\Delta y=\Delta z=\Delta s$ ) the CFL criterion simplifies to

$$
\Delta t \leq \frac{\Delta s}{v_{c} \sqrt{3}} .
$$

At low frequencies $(<10 \mathrm{kHz})$ and high resolution (e.g. $1 \mathrm{~m})$, the amount of timesteps required can easily exceed 100,000. For example, the maximum timestep at $1 \mathrm{~m}$ resolution is $1926 \mathrm{ps}$; the period of a $2000 \mathrm{~Hz}$ signal is $0.5 \mathrm{~ms}$; hence, to model one cycle of this signal, over 250,000 timesteps are need. Furthermore, if mine-scale features are modelled at such resolutions, the size of the computational domain can 
grow very large - on the order of millions of cells.

A method to speed up execution time - assuming there is no air in the model space - is to artificially decrease the speed of light in the various media by increasing their permittivity. The speed of light in a medium is

$$
v_{c}=\frac{1}{\sqrt{\epsilon \mu}}
$$

In general, the ground behaves as a conductor in the VLF frequency range. The predominance of conduction currents versus displacement currents can be assessed through the loss tangent

$$
\tan \delta=\frac{\sigma}{\epsilon \omega}
$$

where $\omega$ is the angular frequency. When the loss tangent is approximately unity, both conduction and displacement currents are of equal importance. When the loss tangent is much greater than unity, conduction currents predominate, and when it is much smaller than unity, displacement currents predominate. At frequencies used in TTE radio, the denominator of the loss tangent is on the order of $10^{-8} \mathrm{~S} / \mathrm{m}$. For this reason, the permittivity of rock is generally of no significance to TTE communications, unless the rock has anomalously low conductivity. Artificially increasing permittivity can therefore significantly improve performance with very minimal effect on the results (Simpson, 2012).

A more generalized way to improve performance is to parallelize the code. In the FDTD technique, the results for timestep at time $t$ depends on the results for the previous timestep at time $t-1$; hence, incrementing has to be done sequentially. However, individual cells can be updated in parallel; the only caveat is that since the grid is staggered both spatially and temporally, only the electric field or the magnetic 
field can be updated in parallel at once. There are many methods to implement a parallel algorithm; for central processing unit (CPU) clusters, communication between the units must be established with a framework such as OpenMP (Open MultiProcessing) or MPI (Message Passing Interface). However, CPU clusters are physically bulky, consume significant amounts of power, and have high startup and maintenance costs. A cheaper and more efficient solution that has gained popularity in recent years is the use of Graphical Processing Units (GPUs) (Inman et al., 2007; Newman, 2013; Stefański et al., 2011, 2013; Toivanen et al., 2011).

The primary purpose of a GPU is to perform calculations to render graphics; these calculations are very similar to the operations performed in FDTD. GPUs are composed of several computational cores, which operate in parallel. An economical means by which to parallelize and accelerate algorithms, general-purpose GPU programming has greatly expanded in recent years. There are two main approaches: CUDA (Compute Unified Device Architecture) and OpenCL (Open Computing Language). CUDA is proprietary and specific to Nvidia graphics cards; OpenCL supports heterogeneous computing and can be used for GPUs or CPUs of most manufacturers. The portability of OpenCL does come at the cost of performance, in comparison to CUDA. Both CUDA and OpenCL implementations involve writing short snippets of code, called kernels, to replace previous single-threaded code.

In this study, an OpenCL-based implementation is used, following Stefański et al. (2011, 2013). A single GPU optimized for general-purpose use was used, which yielded superior computational throughput compared to a similar configuration reported by Stefański et al. (2011, 2013). The GPU used had a clock frequency of $1020 \mathrm{MHz}$ and $6 \mathrm{~GB}$ of memory, and the host CPU had a clock frequency of $2400 \mathrm{MHz}$ and 16 GB RAM. The implementation of the CPML boundary condition had to be carefully considered, as it is more complex than implementing the main field-updating 


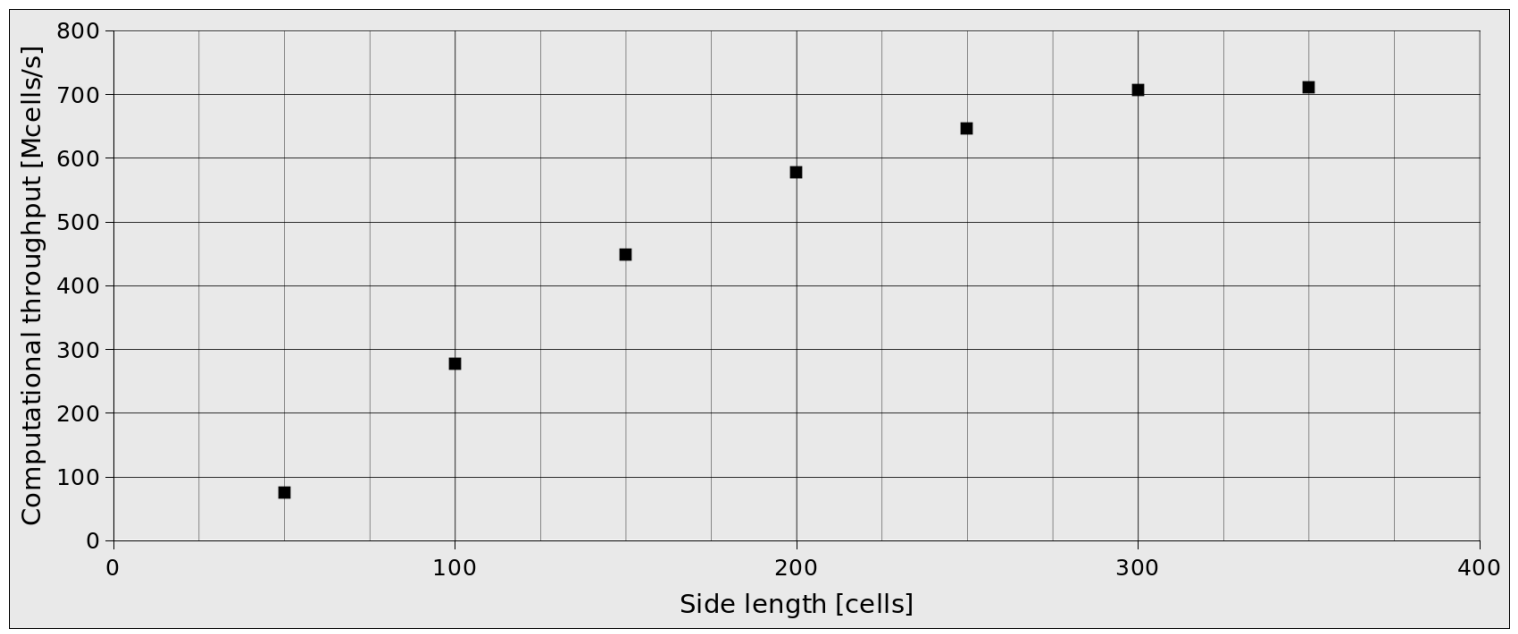

Figure 3.1: Benchmarking of GPU-accelerated FDTD code. A cubic computational domain was used; the side length included a 16-cell CPML. Computational throughput refers to how many cells can be processed per second, which covers several timesteps.

equations (Inman et al., 2007; Toivanen et al., 2011). A scheme similar to one described in Toivanen et al. (2011) was used to apply the CPML; separate kernels were written depending on how many CPML layers were overlapping in any given part of the computational domain.

Benchmarking results for computational throughput with increasing computational domain size are given in Figure 3.1. Note that unlike in the case of a CPU, the efficiency of a GPU increases with memory buffer size. For comparison, a previous proof-of-concept single-thread Fortran code would have taken days to compute the same simulation that now takes hours. 


\subsection{Results and discussion}

\subsubsection{Overview}

Five case studies are presented to demonstrate the capabilities of the new FDTDbased modelling tool. The first three examples have analytic solutions; the latter two are two- and three-dimensional problems, respectively, that can only be solved numerically. The analytic examples have previously been discussed in the literature, whereas cases of a complexity similar to the latter two do not appear to have been attempted in this context (Forooshani et al., 2013; Shope, 1982; Wait and Spies, 1972; Yan et al., 2013; Yarkan et al., 2009; Yenchek et al., 2012).

In TTE radio communication systems, the transmitter is usually a horizontal loop, equivalent to a vertical magnetic dipole (VMD); this configuration tends to optimize the signal-to-noise ratio, as opposed to, for example, grounded dipoles. In the magnetostatic case (i.e. at the DC limit) in free space, the Biot-Savart law for a magnetic field created by a current element applies. For a square loop with side length $u$, the integrated solution is (Griffiths, 1999):

$$
H_{z}=\frac{I u^{2}}{2 \pi\left(z^{2}+\frac{u^{2}}{4}\right) \sqrt{z^{2}+\frac{u^{2}}{2}}},
$$

where $H_{z}$ is the vertical magnetic field, $I$ is the current, and $z$ is the distance along the vertical axis (cf. Figure 3.2). For an AC source, a correction $\xi$ was introduced by Greene (1967):

$$
\xi=\sqrt{1+\left(\frac{\omega z}{c}\right)^{2}},
$$

where $c$ is the speed of light in a vacuum.

For the case where $z \gg u$, equation 3.8 reduces to 


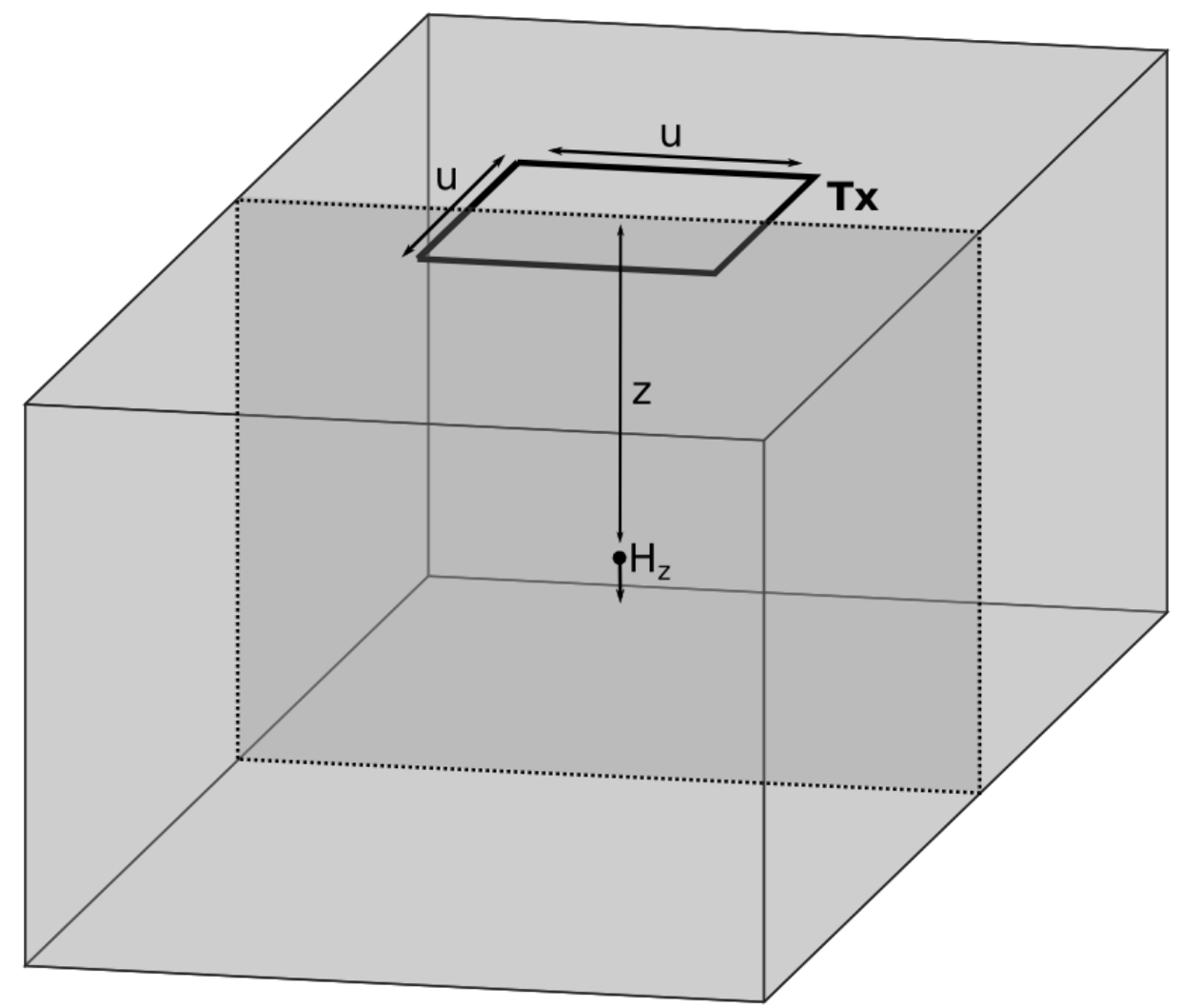

Figure 3.2: Configuration for a square loop (Tx) with side length $u$. A single point of interest is represented at a distance $z$ away from the centre of the loop. Along the loop axis, only the vertical component of the magnetic field is present $\left(H_{z}\right)$, and hence we represent the point of interest as a vector. For Case A (Section 3.5.2), the subsurface is assumed to be free space; for Case $\mathrm{B}$ (Section 3.5.3), it is a homogeneous halfspace. In Case C (Section 3.5.4), the subsurface is layered horizontally. In Cases D and E (Sections 3.5.5 and 3.5.6), the plane drawn in a darker shade of grey represents the cross-section of interest. 


$$
H_{z}=\frac{M}{2 \pi z^{3}}
$$

where $M$ is the dipole moment, which is the product of the number of turns in the transmitter coil $N$, current $I$, and loop area $\mathcal{A}$ :

$$
M=N I \mathcal{A}
$$

For $z \gg u$, there is no difference between the magnetic field of a square loop and that of a circular loop. The latter is usually considered in analytic solutions as it allows the use of cylindrical symmetry in a 1D Earth. However, in TTE radio applications, a square geometry is generally used. For example, in a pillar-and-room coal mine, the transmitter wire is typically wrapped around a rectangular pillar. In addition, a rectangular transmitter can be easily modeled in FDTD; circular geometry is adversely affected by staircasing discretization artifacts.

For propagation outside of free space, equation 3.10 is multiplied by a quality factor $\hat{Q}$ dependent on the material properties and geometric parameters (Shope, 1982; Wait and Spies, 1972). The results for a homogeneous halfspace and a threelayer Earth are provided in appendices 3.8 and 3.9, respectively.

For the FDTD simulations, a cosine-modulated Gaussian function $f$, also called a Morlet wavelet, is used as the current waveform to approximate a typical TTE radio waveform:

$$
f(t)=\cos \left(\omega_{c} t\right) \cdot e^{-\left(\frac{t^{2}}{\tau}\right)}
$$

where $\tau$, a parameter used to describe the width of a Gaussian pulse, is dependent on a central frequency $\omega_{c}$ and a bandwidth $\Delta f$ : 


$$
\tau=\frac{2 \sqrt{2.3}}{\pi \Delta f}
$$

In the frequency domain, this function is bell-shaped, with a peak at the central frequency and the width equivalent to the bandwidth:

$$
F(\omega)=\frac{\tau \sqrt{\pi}}{2}\left(e^{-\frac{\tau^{2}\left(\omega-\omega_{c}\right)}{4}}+e^{-\frac{\tau^{2}\left(\omega+\omega_{c}\right)}{4}}\right) .
$$

The excitation function are given in Figure 3.3 (Elsherbeni and Demir, 2009).

\subsubsection{Case A: Biot-Savart law}

The first case considered was signal propagation in free space as described by the Biot-Savart law. This situation was the easiest to accurately describe analytically; a square loop was used in the expression and the $\mathrm{AC}$ correction (equation 3.9) was applied. The results and exact case parameters are given in Figure 3.4.

There is a very high degree of agreement between analytic and FDTD results - the percent difference is on the order of $0.01 \%$. This close agreement is due to two factors. First, the analytic solution precisely describes the transmitter geometry. Second, a very large air buffer and thick CPML is present around the loop, which greatly reduces inaccuracies arising from the numeric reflections at domain edges. However, the execution time for such a large computational domain for the 500,000 timesteps required for this frequency is approximately $5 \frac{1}{2}$ hours, which can be prohibitively long to run routinely. Furthermore, for a realistic TTE simulation, the high uncertainty in the material parameters - especially the conductivity - , reduces any accuracy gained with a big material buffer. 


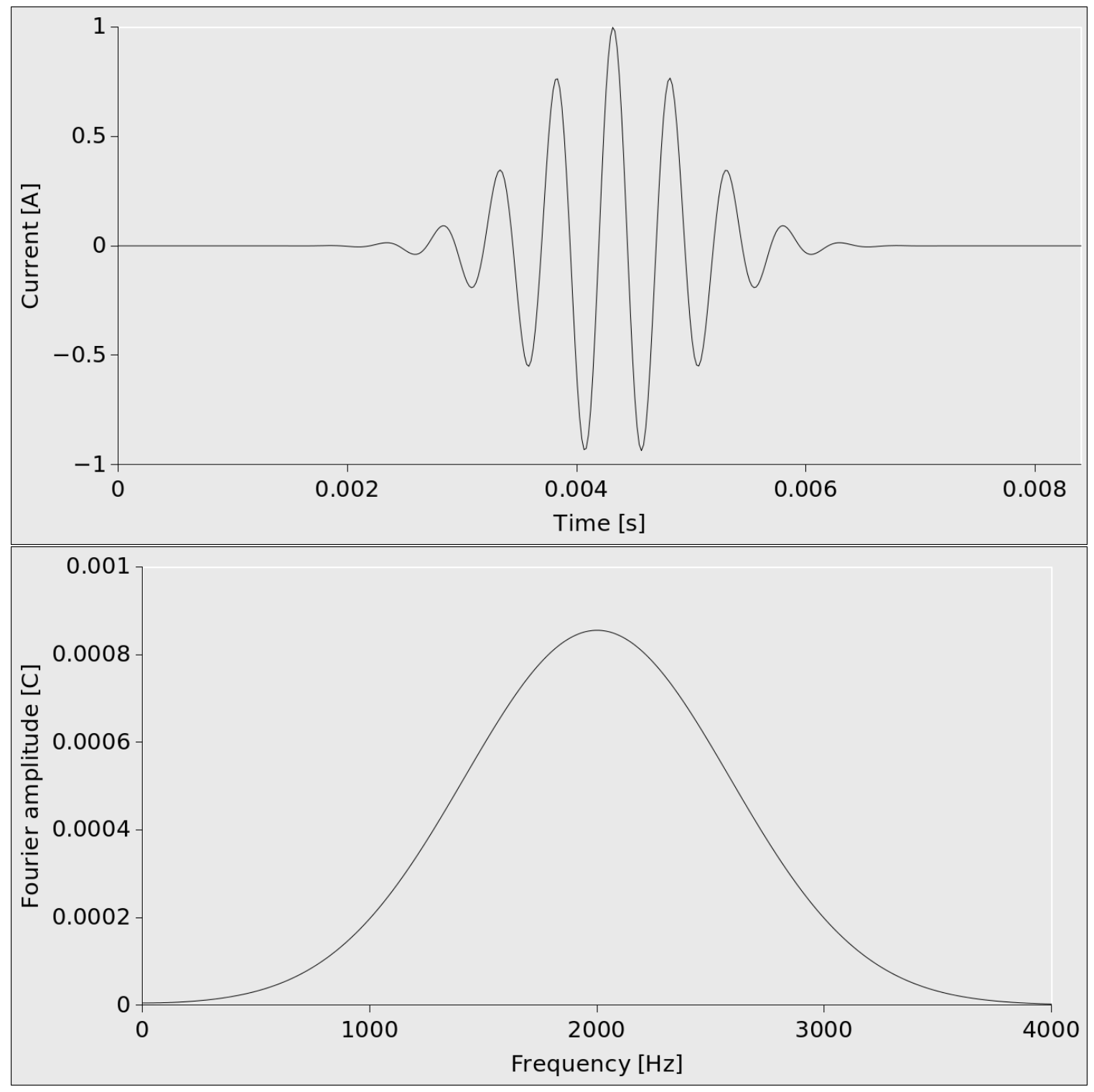

Figure 3.3: Time-domain (top) and frequency-domain (bottom) graphs of the cosine-modulated Gaussian function used as the excitation waveform for the FDTD simulations. These graphs are for a central frequency of $2000 \mathrm{~Hz}$ and a bandwidth of $1000 \mathrm{~Hz}$; these parameters were used in cases D and E. 


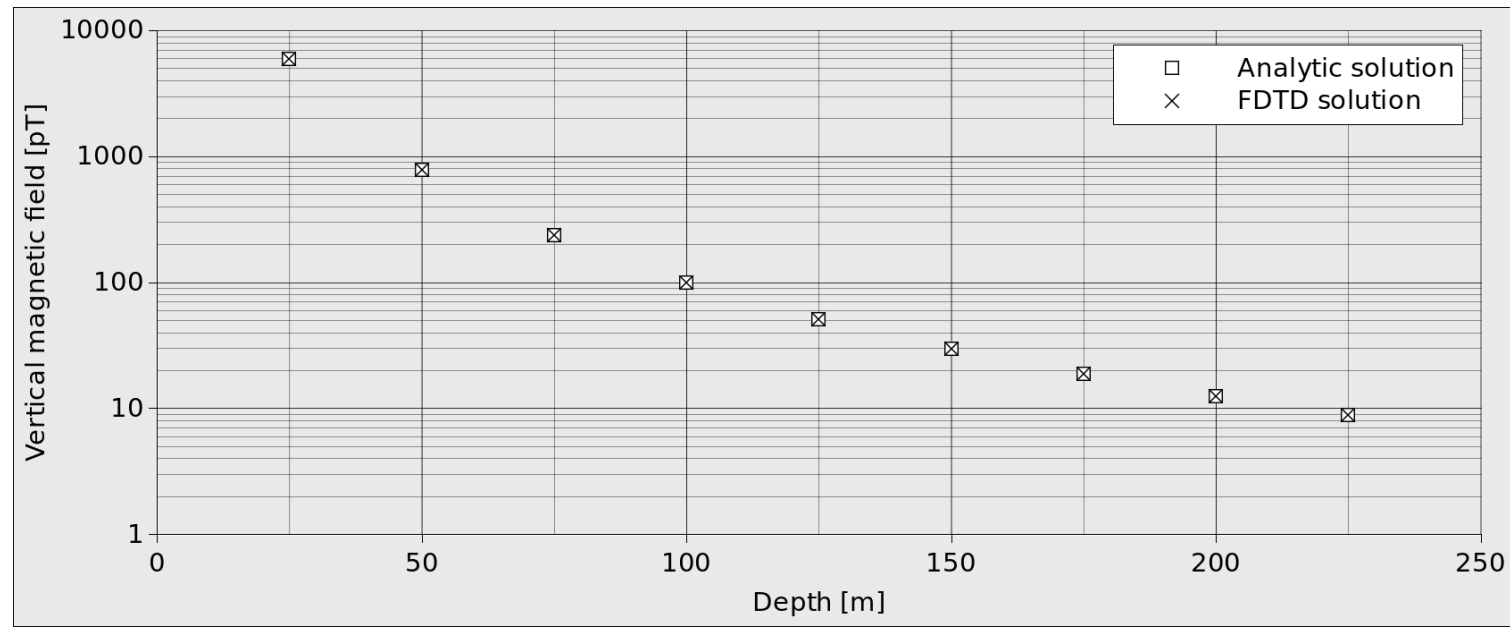

\begin{tabular}{|l|l|}
\hline Domain size & $300 \mathrm{~m} \times 300 \mathrm{~m} \times 300 \mathrm{~m}$ \\
\hline CPML thickness & 16 cells \\
\hline Cell size & $1 \mathrm{~m}$ \\
\hline Loop size & $10 \mathrm{~m} \times 10 \mathrm{~m} ;$ VMD in centre of domain at $\mathrm{z}=50 \mathrm{~m}$ \\
\hline Current & $5 \mathrm{~A}$ \\
\hline Frequency & $10.0 \mathrm{kHz}$ \\
\hline Bandwidth & $5.0 \mathrm{kHz}$ \\
\hline Geometry & Free space \\
\hline
\end{tabular}

Figure 3.4: Comparison of the vertical magnetic field in free air versus increasing depth along the transmitter axis obtained from the Biot-Savart law (analytic) and FDTD. 


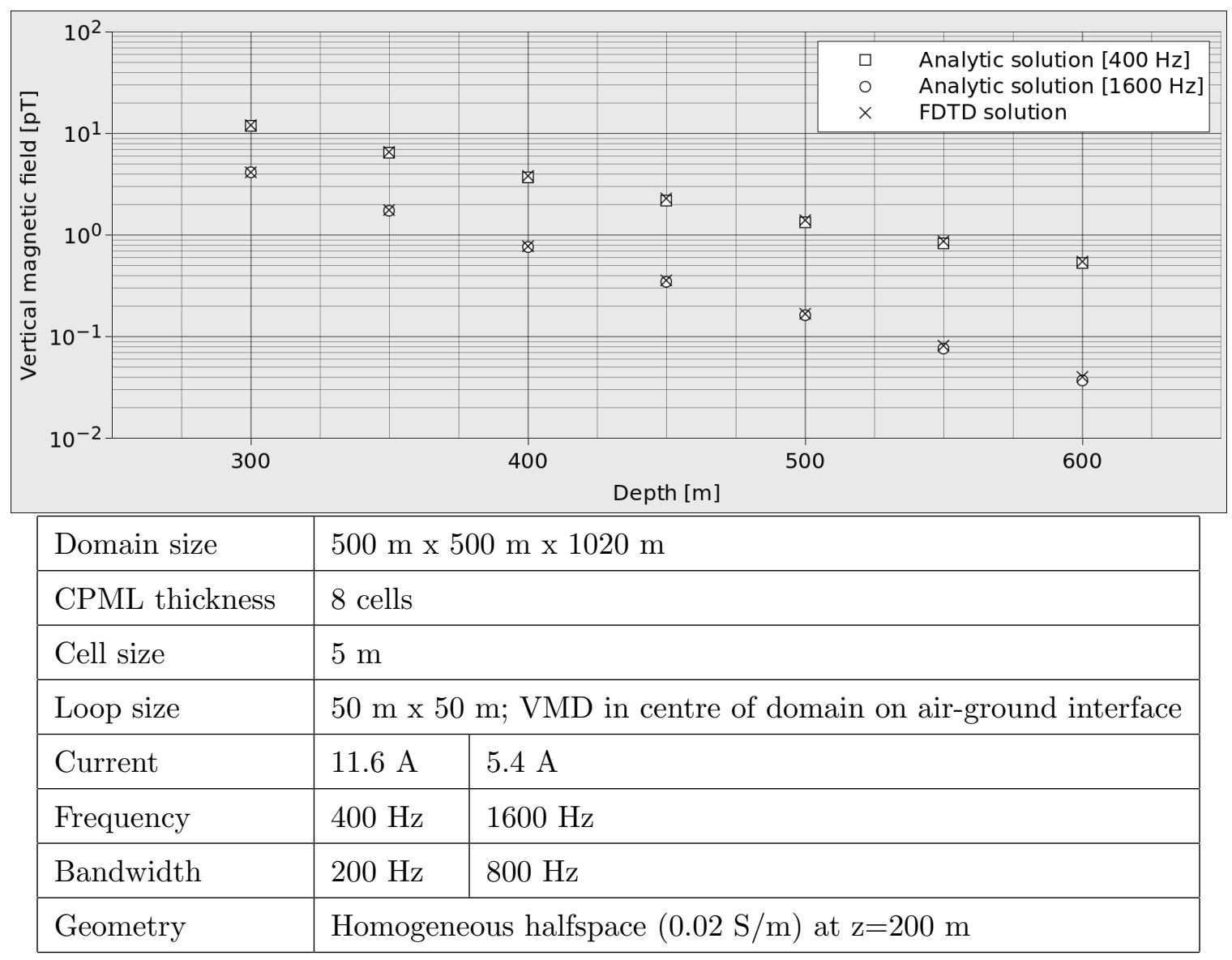

Figure 3.5: Comparison of the vertical magnetic field in a homogeneous halfspace versus increasing depth along the transmitter axis obtained from an analytic solution (Wait and Spies, 1972) and FDTD, for two different frequencies. The vertical magnetic field strength has been normalized to a current of $1 \mathrm{~A}$. Field strength at $400 \mathrm{~Hz}$ is greater at the same depth compared to that at $1600 \mathrm{~Hz}$.

\subsubsection{Case B: Homogeneous halfspace}

The second analytically solvable case considered was transmission in a homogeneous halfspace; the expression, per Wait and Spies (1972), is given in Appendix 3.8. The analytic results were previously verified numerically by Yan et al. (2013) using a method-of-moments solver, in the context of TTE communications.

Per Figure 3.5, there is close agreement between the analytic and numeric results at both $400 \mathrm{~Hz}$ and $1600 \mathrm{~Hz}$. As expected, the values for $400 \mathrm{~Hz}$ are greater than those 
for $1600 \mathrm{~Hz}$, as lower-frequency signals attenuate less during propagation. A source of error, albeit slight, is geometry; the FDTD loop is a square, whereas the analytic result was calculated for a circular loop of equivalent area. The boundary conditions also contribute to the discrepancy; by trial-and-error, it has been observed that slight changes to CPML parameters or thickness can cause minor differences in the output on the scale of the deviations seen in Figure 3.5. In the context of modelling TTE communications, differences of a few percent are negligible in practice because of the typically poor control on conductivity estimates.

\subsubsection{Case C: Layered Earth}

The final case with an analytic solution considered is a three-layer Earth model (Figure 3.6). The expression, per Shope (1982), is given in Appendix 3.9; note that this solution is applicable only for a buried transmitter. A stratigraphic sequence varying in one dimension is modelled, with conductivities typical of sedimentary rocks. A transmitter is placed underground with the receiver at the surface.

Overall, the modelling tool accurately replicated results from analytic expressions. The loss of signal strength with increasing frequency due to the expected attenuation by a conductive material, is clearly seen. There are, however, some small deviations between the analytic and numeric results. As in Case B, the geometric difference between a square FDTD loop and a circular analytic loop contributes to the difference, but it is likely not the prime source of error.

In this case, two other sources of error have an impact on the results. First, as with

Case B, slight changes to the boundary conditions will shift values by as much as a few percent. This effect is perhaps more noticeable in this case study, because the material buffer around the transmitter loop is smaller. Similarly, the bottom layer (Rock 3) is thin, and may insufficiently approximate an infinite halfspace. Furthermore, as this 


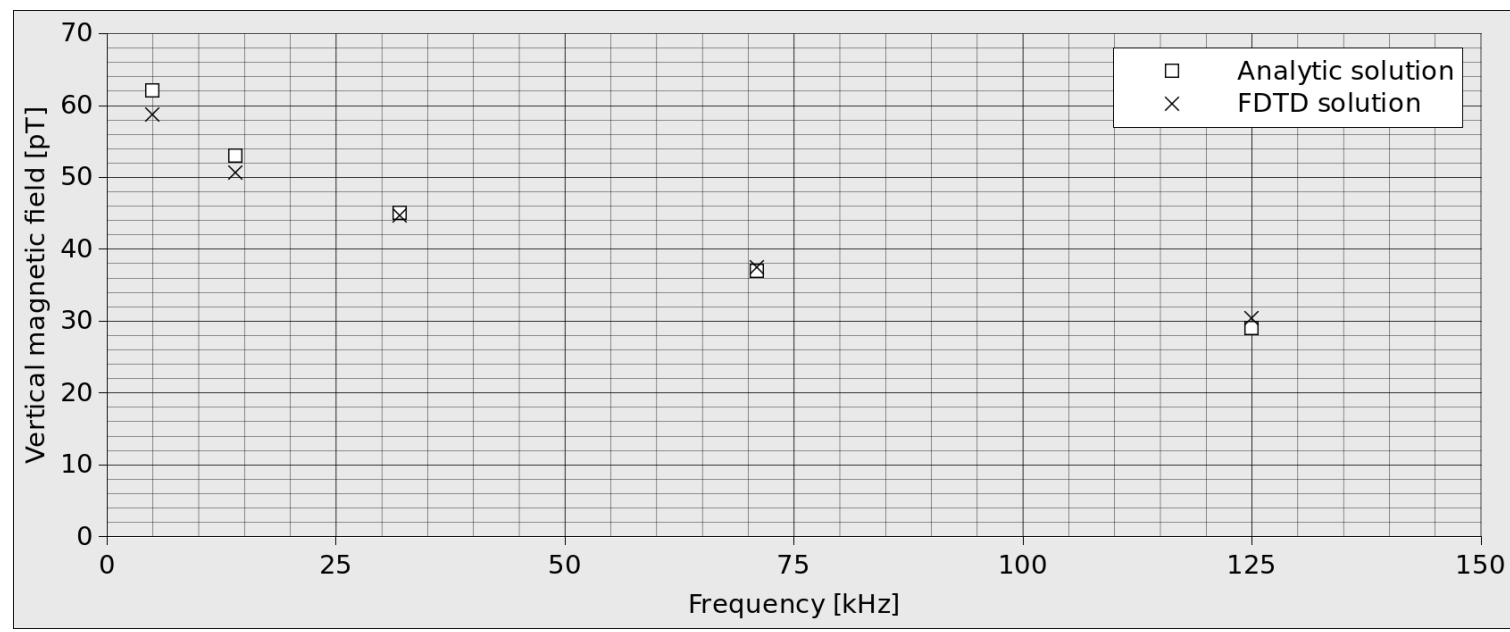

\begin{tabular}{|c|c|c|c|c|c|}
\hline Domain size & \multicolumn{5}{|c|}{$50 \mathrm{~m} \times 50 \mathrm{~m} \times 150 \mathrm{~m}$} \\
\hline CPML thickness & \multicolumn{5}{|l|}{8 cells } \\
\hline Cell size & \multicolumn{5}{|l|}{$1 \mathrm{~m}$} \\
\hline Loop size & \multicolumn{5}{|c|}{$10 \mathrm{~m} \times 10 \mathrm{~m} ; \mathrm{VMD}$ in the Earth at $\mathrm{z}=120 \mathrm{~m}$} \\
\hline Receiver location & \multicolumn{5}{|c|}{ Air-ground interface at $\mathrm{z}=20 \mathrm{~m}$, along Tx axis } \\
\hline Current & \multicolumn{5}{|l|}{$5 \mathrm{~A}$} \\
\hline Frequency & $5.0 \mathrm{kHz}$ & $14.0 \mathrm{kHz}$ & $32.0 \mathrm{kHz}$ & $71.0 \mathrm{kHz}$ & $125.0 \mathrm{kHz}$ \\
\hline Bandwidth & $2.5 \mathrm{kHz}$ & $7.0 \mathrm{kHz}$ & $16.0 \mathrm{kHz}$ & $35.5 \mathrm{kHz}$ & $62.5 \mathrm{kHz}$ \\
\hline \multirow{4}{*}{ Geometry } & Air & \multicolumn{4}{|l|}{$0-20 \mathrm{~m}$} \\
\hline & Rock 1 & \multicolumn{4}{|c|}{$0.0001 \mathrm{~S} / \mathrm{m} ; 20-110 \mathrm{~m}$} \\
\hline & Rock 2 & \multicolumn{4}{|c|}{$0.01 \mathrm{~S} / \mathrm{m} ; 110-130 \mathrm{~m}$} \\
\hline & Rock 3 & \multicolumn{4}{|c|}{$0.25 \mathrm{~S} / \mathrm{m} ; 130-150 \mathrm{~m}$} \\
\hline
\end{tabular}

Figure 3.6: Comparison of the vertical magnetic field in a three-layer Earth versus frequency obtained from an analytic solution (Shope, 1982) and FDTD. 
layer is located close to the transmitter, the numeric artifacts may contribute more to the magnetic field at the surface.

\subsubsection{Case D: Faulted coal seam}

A faulted coal seam is considered in Figure 3.7. A coal seam with a thickness of $8 \mathrm{~m}$ has been normally faulted; the hanging wall has been downthrown by $5 \mathrm{~m}$. The coal is significantly more conductive than the host rock, and is presumed to impede TTE radio transmissions. An analytic solution does not exist for this scenario, given its two-dimensional nature. Coal-bearing geology does not always consists of uniform flat-lying beds such as in Case C; it often exhibits significant structural features.

The FDTD simulation shows that the magnetic field diffuses down along the fault. As expected, there is a marked reduction in field strength below the coal seam, as this conductive layer absorbs the transmitted EM energy by forming eddy currents. The reduction is asymmetric and depends on the vertical thickness of the coal seam. Local minima are observed within the base of the coal layer at points farthest away from the transmitter; however, the field strength in the host rock directly below is greater than in the coal seam.

A strength of FDTD modelling is that, as a time-domain technique, a time series of field behavior can be sampled. We can therefore visualize the propagation of the magnetic field as an animation. A few frames from this animation are given sequentially in Figure 3.8. A quarter period is shown, from the null to the peak of a crest (cf. Figure 3.3). The diffusion of the signal into the Earth is clearly visible, as is its attenuation as it reaches the conductive coal seam. At $4.25 \mathrm{~ms}$ and $4.30 \mathrm{~ms}$, a local minimum is seen propagating down; this minimum corresponds to the signal null which occurs when the field reverses direction. By $4.42 \mathrm{~ms}$, we see that the peak of the crest of the field has passed and signal amplitude has begun to decrease. 

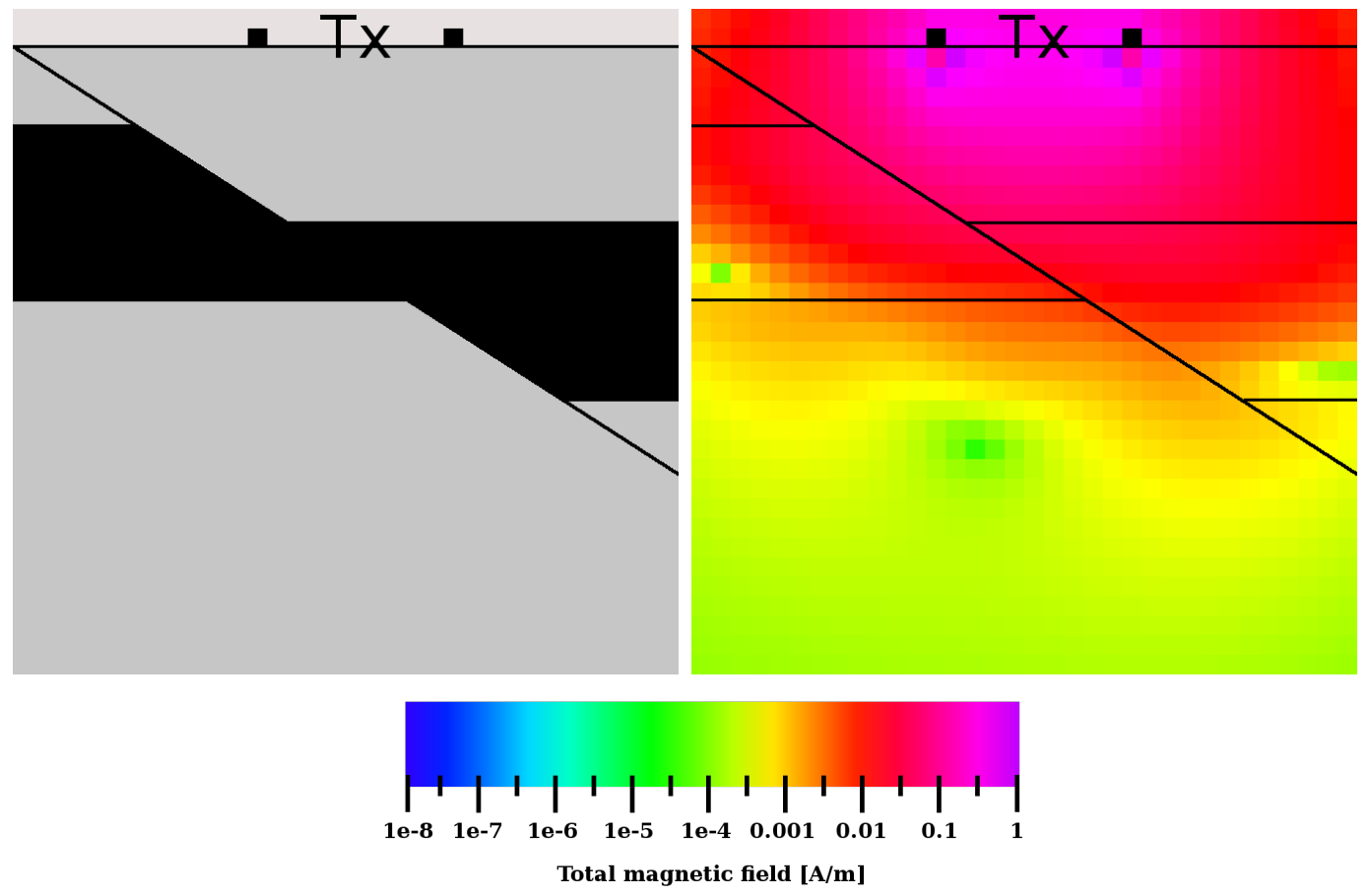

\begin{tabular}{|c|c|c|}
\hline Domain size & \multicolumn{2}{|c|}{$50 \mathrm{~m} \times 50 \mathrm{~m} \times 50 \mathrm{~m}$} \\
\hline CPML thickness & \multicolumn{2}{|l|}{8 cells } \\
\hline Cell size & \multicolumn{2}{|l|}{$1 \mathrm{~m}$} \\
\hline Loop size & \multicolumn{2}{|c|}{$10 \mathrm{~m} \times 10 \mathrm{~m}$; VMD in centre of domain on air-ground interface } \\
\hline Current & \multicolumn{2}{|l|}{$5 \mathrm{~A}$} \\
\hline Frequency & \multicolumn{2}{|l|}{$2000 \mathrm{~Hz}$} \\
\hline Bandwidth & \multicolumn{2}{|l|}{$1000 \mathrm{~Hz}$} \\
\hline \multirow{3}{*}{ Geometry } & Air [light grey] & $0 \mathrm{~S} / \mathrm{m} ; 10 \mathrm{~m}$ thick \\
\hline & Rock [grey] & $0.0001 \mathrm{~S} / \mathrm{m}$; variable thickness \\
\hline & Coal [black] & $10 \mathrm{~S} / \mathrm{m} ; 8 \mathrm{~m}$ thick \\
\hline
\end{tabular}

Figure 3.7: Total magnetic field $\left(H_{m}=\sqrt{{H_{x}}^{2}+{H_{y}}^{2}+H_{z}{ }^{2}}\right)$ in faulted coal-bearing stratigraphy, sampled at a timestep $4.81 \mathrm{~ms}$ into the simulation. The rock units extend uniformly into the third dimension, thus giving only $2 \mathrm{D}$ variation. The stratigraphic cross-section (L) and magnetic field cross-section (R) are $34 \mathrm{~m} \mathrm{x}$ $34 \mathrm{~m}$; the eight CPML cells (1 $\mathrm{m}$ each) on both sides have been cropped out. Note the logarithmic colormap. The black squares represent the cross-sectional cut of the transmitter loop. 


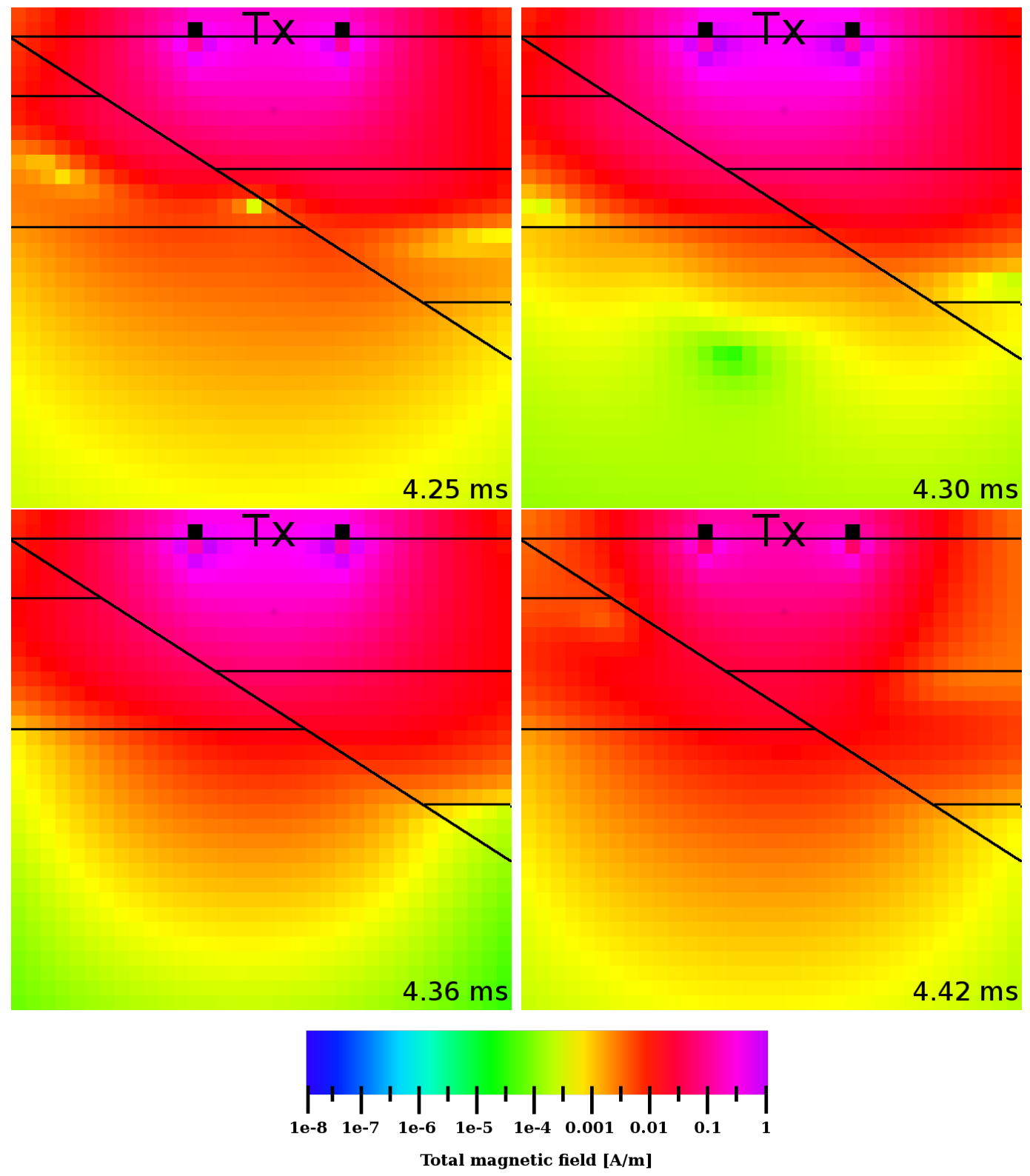

Figure 3.8: Magnetic field propagation through the model shown in Figure 3.7 versus time; Figure 3.7 corresponds to $4.81 \mathrm{~ms}$. The field is sampled (left-toright) at $4.25 \mathrm{~ms}, 4.30 \mathrm{~ms}, 4.36 \mathrm{~ms}$, and $4.42 \mathrm{~ms}$; the excitation waveform is given in Figure 3.3. 
Such modelling work has important practical implications. A TTE receiver deployed in the vicinity of a major fault will pick up more signal, as it is leaking down the fault. Although the coal does clearly attenuate the signal, there is still substantial signal strength at depth. These detailed results can suggest an optimal location to place an underground transmitter-receiver pair; in this case, below the coal seam, there remains some appreciable signal strength along the fault.

\subsubsection{Case E: Salt diapir}

The final case study concerns a propagation through salt diapir. This example was chosen to illustrate the 3D capabilities of the new modelling tool. In this case, there is $3 \mathrm{D}$ variation; a hemispherical body is superimposed upon a layered Earth. This case study also demonstrated that the modelling tool can accurately handle objects that do not have a rectangular geometry.

While not a typical geologic setting for TTE radio, the technology has been recently demonstrated in evaporitic geology, specifically in a trona mine and a potash mine. Evaporites are typically resistive; on the other hand, saline water is an excellent electrolytic conductor (Parkhomenko, 1967). A salt diapir is modelled in Figure 3.9, with a highly conductive cap (black) above the tabular salt layer and its associated diapir (dark grey). This conductive cap is assumed to have formed by leaching of the salt. Even though the cap is very thin $(2 \mathrm{~m})$, it acts as a barrier to the propagation of radio signal and a reduction in the magnetic field by 1-2 orders of magnitude is observed below. However, the presence of the highly resistive salt body permits more energy to reach into and slightly below the diapir. This example shows that even though an evaporite layer per se would be a favorable setting for TTE communications, leaching of the evaporitic layer greatly attenuates the signal.

An animation (Figure 3.10) was sampled at the same times as for Case D (note 

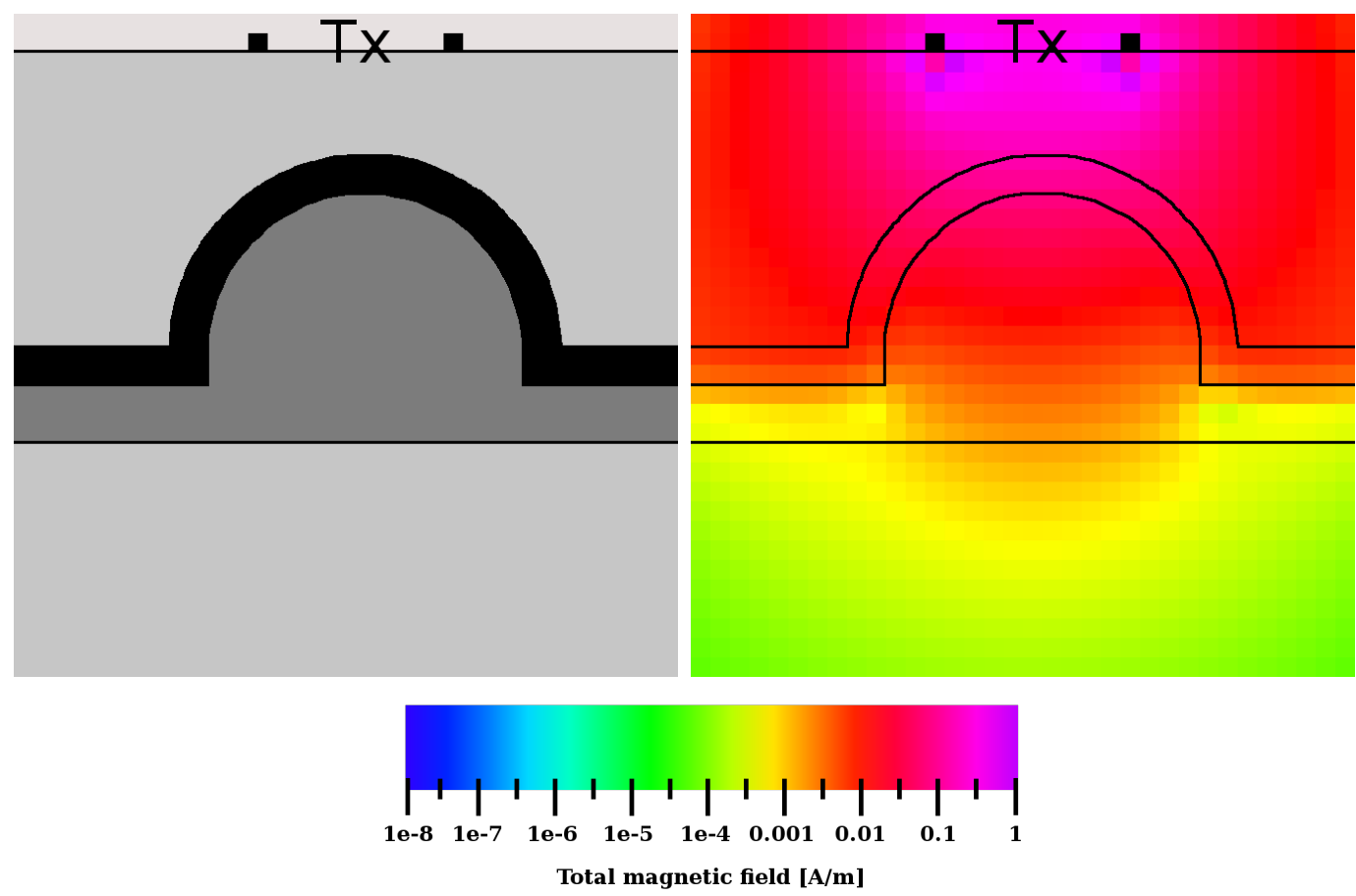

\begin{tabular}{|c|c|c|}
\hline Domain size & \multicolumn{2}{|l|}{$50 \mathrm{~m} \times 50 \mathrm{~m} \times 50 \mathrm{~m}$} \\
\hline CPML thickness & \multicolumn{2}{|l|}{8 cells } \\
\hline Cell size & \multicolumn{2}{|l|}{$1 \mathrm{~m}$} \\
\hline Loop size & \multicolumn{2}{|c|}{$10 \mathrm{~m} \times 10 \mathrm{~m}$; VMD in centre of domain on air-ground interface } \\
\hline Current & \multicolumn{2}{|l|}{$5 \mathrm{~A}$} \\
\hline Frequency & \multicolumn{2}{|l|}{$2000 \mathrm{~Hz}$} \\
\hline Bandwidth & \multicolumn{2}{|l|}{$1000 \mathrm{~Hz}$} \\
\hline \multirow{4}{*}{ Geometry } & Air [light grey] & $0 \mathrm{~S} / \mathrm{m} ; 10 \mathrm{~m}$ thick \\
\hline & Rock [grey] & $\begin{array}{l}0.0001 \mathrm{~S} / \mathrm{m} ; 15 \mathrm{~m} \text { thick above } \\
\text { and } 18 \mathrm{~m} \text { thick below main salt } \\
\text { layer }\end{array}$ \\
\hline & Wet saline rock [black] & $\begin{array}{l}10 \mathrm{~S} / \mathrm{m} ; 2 \mathrm{~m} \text { thick around main } \\
\text { salt layer and diapir }\end{array}$ \\
\hline & Salt [dark grey] & $\begin{array}{l}10^{-7} \mathrm{~S} / \mathrm{m} \text {; diapir and } 5 \mathrm{~m} \text { thick } \\
\text { tabular layer }\end{array}$ \\
\hline
\end{tabular}

Figure 3.9: Total magnetic field $\left(H_{m}=\sqrt{{H_{x}}^{2}+{H_{y}}^{2}+{H_{z}}^{2}}\right)$ in salt-bearing stratigraphy, sampled at a timestep $4.81 \mathrm{~ms}$ into the simulation. The tabular units vary only with depth, whereas the salt diapir is effectively a hemispherical body in three dimensions, which gives overall 3D variation. The stratigraphic crosssection (L) and magnetic field cross-section (R) are $34 \mathrm{~m} \mathrm{x} 34 \mathrm{~m}$; the eight CPML cells (1 m each) on both sides have been cropped out. Note the logarithmic colormap. 


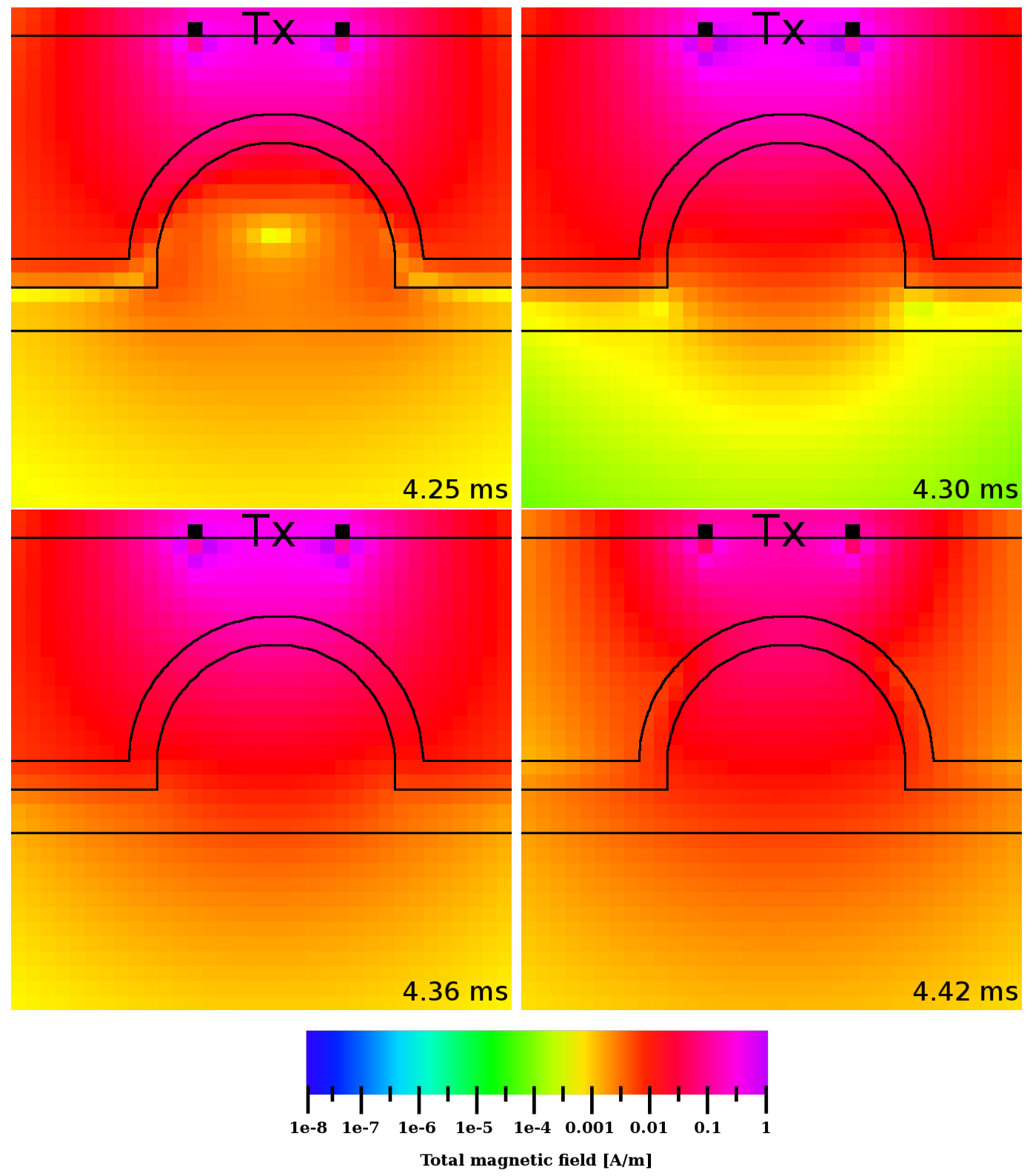

Figure 3.10: Magnetic field propagation through the model shown in Figure 3.9 versus time; Figure 3.9 corresponds to $4.81 \mathrm{~ms}$. The field is sampled (left-toright) at $4.25 \mathrm{~ms}, 4.30 \mathrm{~ms}, 4.36 \mathrm{~ms}$, and $4.42 \mathrm{~ms}$; the excitation waveform is given in Figure 3.3. 
also that identical sources were used for Cases D and E). At $4.25 \mathrm{~ms}$, we see the signal null in the diapir itself; at $4.30 \mathrm{~ms}$, it migrates below the evaporitic layer. At $4.36 \mathrm{~ms}$, the maximum signal strength at the source is observed, and the magnetic field no longer appears deformed by the salt diapir. By $4.42 \mathrm{~ms}$, the field has begun to reverse, although a local maximum has developed in the salt diapir itself because the signal is less attenuated there, due to the extremely low conductivity of the salt.

This example demonstrates that three-dimensional geometry can be reliably modelled. The results shown in Figures 3.9 and 3.10 confirm that a highly conductive layer with no interruptions will greatly attenuate a TTE signal, although the presence of a very low conductivity body will allow the signal to persist further.

\subsection{Conclusion}

A new FDTD-based tool was developed to perform forward modelling of TTE radio signal propagation. Modelling has shown that in realistic geologic scenarios, the signal can vary by two orders of magnitude within several metres, depending on the transmitter and receiver locations relative to various geologic features. This modelling tool is invaluable in determining the optimal placement of a transmitter and receiver pair to obtain the best signal-to-noise ratio.

The new tool uses the CPML boundary condition to absorb energy at the edges of the model domain, and employs GPU acceleration to dramatically reduce execution time. This paper features five case studies where the domain was on the order of $10^{5}-10^{7}$ cells. At TTE radio frequencies $(400-9000 \mathrm{~Hz})$, solutions were obtained in a reasonable few minutes or hours, which would have previously taken several days. FDTD is scalable: for larger-scale problems, such as an entire mine on the scale of several hundreds of metres, the cell size can be increased, which would keep the overall 
domain size reasonable.

Comparisons were made with three cases that have an analytic solution; two case studies were shown for geologies relevant to TTE communications that cannot be solved analytically. The new tool generated results with minimal deviation compared to the analytic predictions. The main source of error is the handling of boundaries. The finite computational domain introduces errors in the results. The CPML and air/material buffers help to improve the results. As seen from Case A, however, very thick buffers and CPML, along with matching geometry, are needed to obtain a match within a fraction of a percent.

When modelling realistic TTE communications scenarios, the uncertainty in the conductivities of the various geologic formations contribute much more to the overall uncertainty than do the boundary issues. FDTD is extremely flexible with regards to defining the geometry and material properties of a model - if desired, every cell could be assigned a different conductivity. Forward modelling could therefore be improved in the future by integrating site-specific conductivity information.

\subsection{Acknowledgments}

This research project is funded by the Natural Sciences and Engineering Research Council of Canada (NSERC) and Vital Alert Communications, Inc., through an Industrial Postgraduate Scholarship to M. Ralchenko. 


\subsection{Appendix I: Homogeneous halfspace}

The vertical magnetic field $H_{z}$ generated by a horizontal loop placed on the interface between air and a homogeneous halfspace with conductivity $\sigma$ is expressed as

$$
H_{z}=\frac{M}{2 \pi z^{3}} \hat{Q}
$$

where $\hat{Q}$ is a factor dependent on the material properties and geometric parameters, $M$ is the dipole moment, and $z$ is the depth. The dipole moment is the product of the number of turns $N$, the current $I$, and loop area $\mathcal{A}$ :

$$
M=N I \mathcal{A}
$$

Geometrical parameters are normalized with respect to the number of skin depths $\zeta$ from the dipole on the air-ground interface to the underground receiver. A circular loop of radius $a$ is assumed, which permits the use of cylindrical symmetry. For magnetic permeability $\mu$ and angular frequency $\omega$, we obtain

$$
\zeta=(\mu \omega \sigma)^{1 / 2} z
$$

and

$$
A=a / z
$$

Finally, the expression for $\hat{Q}$ is obtained

$$
\hat{Q}=\int_{0}^{\infty} \frac{J_{1}(A x)}{A x / 2} \cdot \frac{x^{3}}{\sqrt{x^{2}+i \zeta^{2}}+x} \cdot e^{-\left(x^{2}+i \zeta^{2}\right)^{1 / 2}} d x
$$

where $J_{1}$ is the Bessel function of the first kind. 


\subsection{Appendix II: Three-layer Earth}

A relation is defined for a buried vertical magnetic dipole in a geology with three layers with conductivities $\sigma_{1}, \sigma_{2}$, and $\sigma_{3}$ (Shope, 1982). Following the convention of Wait and Spies (1972), the vertical magnetic field $H_{z}$ generated is expressed as

$$
H_{z}=\frac{M}{2 \pi z^{3}} \hat{Q}
$$

where $\hat{Q}$ is a factor dependent on the material properties and geometric parameters, $M$ is the dipole moment, and $z$ is the depth. The dipole moment is the product of the number of turns $N$, the current $I$, and loop area $\mathcal{A}$ :

$$
M=N I \mathcal{A}
$$

An infinitesimal (non-physical) dipole is assumed.

Geometrical parameters are normalized with respect to the number of skin depths $\zeta$ to the buried dipole. Let $b$ represent the distance from the dipole to the interface of layers 1 and 2, and $c$ represent the distance from the dipole to the interface of layers 2 and 3. Hence, for magnetic permeability $\mu$ and angular frequency $\omega$, we obtain

$$
\begin{gathered}
\zeta=\left(\mu \omega \sigma_{2}\right)^{1 / 2} z, \\
B=b / z,
\end{gathered}
$$

and

$$
C=c / z
$$

The conductivities of layers 1 and 3 are normalized to that of layer 2 : 


$$
S_{12}^{2}=\sigma_{1} / \sigma_{2}
$$

and

$$
S_{23}^{2}=\sigma_{3} / \sigma_{2}
$$

Transmission and reflection coefficients are obtained based on the wavenumbers $k$ of each layer:

$$
T_{i j}=\frac{2 k_{i}}{k_{i}+k_{j}}
$$

and

$$
R_{i j}=\frac{k_{i}-k_{j}}{k_{i}+k_{j}}
$$

where

$$
\begin{gathered}
k_{1}=\frac{1}{z}\left(x^{2}+i \zeta^{2} S_{12}^{2}\right)^{1 / 2}, \\
k_{2}=\frac{1}{z}\left(x^{2}+i \zeta^{2}\right)^{1 / 2}
\end{gathered}
$$

and

$$
k_{3}=\frac{1}{z}\left(x^{2}+i \zeta^{2} S_{23}^{2}\right)^{1 / 2}
$$

Finally, we obtain the expression for $\hat{Q}$ :

$$
\begin{gathered}
\hat{Q}=\frac{1}{2} \int_{0}^{\infty} \frac{x^{2} T_{01} T_{12}\left(1+R_{23} e^{-2 C\left(x^{2}+i \zeta^{2}\right)^{1 / 2}}\right) e^{-\left(x^{2}+i \zeta^{2}\right)^{1 / 2}(1+B)}}{R_{23} e^{-2 C\left(x^{2}+i \zeta^{2}\right)^{1 / 2}}\left(R_{01} e^{-2\left(x^{2}+i \zeta^{2} S_{12}\right)^{1 / 2}}+R_{12} e^{-2 B\left(x^{2}+i \zeta^{2}\right)^{1 / 2}}\right)} \cdots \\
\cdots \frac{e^{-2 B\left(x^{2}+i \zeta^{2}\right)^{1 / 2}} d x}{T_{01} T_{12} x^{2}\left(1+R_{23} e^{-2 C\left(x^{2}+i \zeta^{2}\right)^{1 / 2}}\right)}
\end{gathered}
$$




\section{Chapter 4}

\section{Estimating subsurface conductivity while optimizing through-the-Earth radio communications using a wideband electromagnetic signal}

\subsection{Abstract}

The propagation of a through-the-Earth (TTE) radio signal depends chiefly on the subsurface conductivity distribution. A method was developed to determine the conductivity distribution of a mine overburden using TTE radio transmission data. At multiple locations in an underground test mine, we recorded a wideband electromagnetic signal, spanning a frequency range of 500-8500 Hz, transmitted from a vertical magnetic dipole at the surface. A four-layer conductivity model that accounts for topography was fitted to the observed data. The difference between the calculated and observed attenuation for each transmitter-receiver pair, for each frequency of interest, was minimized, using a finite-difference time-domain (FDTD) forward modeller. The presence of several railway tracks in the mine affected the recordings, as 
the signal tends to couple to elongated metallic objects. Further work is needed to quantitatively account for this effect, which is of interest equally for TTE radio communications, where this coupling improves signal range, and for electromagnetic methods in exploration geophysics, where such coupling is either noise or confirmation of a target.

\subsection{Introduction and objective}

Through-the-Earth (TTE) radio has been proposed for wireless underground communications, by sending electromagnetic signals in the extremely low to very low frequency ranges; in practice, frequencies between 400 and $9000 \mathrm{~Hz}$ are used. Applications of this technology have been suggested in mine safety, infrastructure such as high-rise buildings or civilian tunnels, the military, and even wireless detonation and activation. A TTE radio system can provide a communication link when conventional wired systems have been rendered unusable. A typical TTE radio system is a pair of terminals, each equipped with a transmitter $(\mathrm{Tx})$ and receiver $(\mathrm{Rx})$.

The main objective of this research is to demonstrate the viability of using a wideband through-the-Earth radio signal to obtain site-specific conductivity information. In particular, we seek to determine the conductivity structure of a mine overburden through which a TTE radio link is to be established. In our previous work (Ralchenko et al., 2015), we simulated TTE radio signal propagation using the finite-difference time-domain (FDTD) method, for an arbitrary transmitter in an arbitrary geologic structure. This modelling approach assumed a conductivity distribution based on a literature review for similar rock types, which may not be representative of the in-situ properties of the rocks of a specific site. Given the conductivity structure, a wealth of practical information is obtained: an optimal transmission frequency can 
be estimated; the relative positions of the transmitter and receiver can be optimized; or, the maximum range, both vertical and lateral, can be predicted.

\subsection{Literature review}

\subsubsection{Electromagnetic methods}

In TTE communication, we are only concerned with the impact of the properties of the overburden between the surface and a specific mine level. Performing conductivity measurements on hand samples or drill core is not a viable approach. First, these samples are not a representative element of the overall overburden. Even if they were, such a process is time consuming: not only do samples have to be processed (e.g. cutting, pore water injection), but the measured conductivity takes days to weeks to stabilize after pore water is added, during which it varies by orders of magnitude (Enkin et al., 2012), which introduces an unacceptable level of error. Conventional inductive geophysical techniques, such as the audio magnetotelluric (AMT) or the time-domain electromagnetic (TDEM) methods, will suffer from strong cultural noise typical of mining environments and terrain accessibility issues. The DC resistivity technique becomes impractical if test locations have a significant amount of area paved or otherwise built upon, thus prohibiting planting electrodes in the ground. For example, underground coal mines, which are significant potential settings for TTE radio deployment, can have a very large lateral extent and considerable surface infrastructure, and be located in difficult terrain, making it unsafe or otherwise impossible to access continuous sections of the surface. Any large-scale surface geophysical program will be logistically unfeasible. Finally, conventional EM methods are not restricted to only the overburden between the mine level and the surface, which is the region of interest for TTE radio transmissions. 


\subsubsection{Radio imaging method}

In the late 1980s, the radio imaging method (RIM) was proposed for tomographic imaging of sections between two boreholes (Thomson and Hinde, 1993). In contrast to TTE radio, RIM operates at the medium frequency (MF) range (0.1-10 MHz). RIM measures the attenuation between the boreholes; ray paths of higher attenuation correspond to areas of high conductivity. Interpretation of data is complicated - at such frequencies, neither conduction nor displacement currents can be neglected. Both FDTD (Yu et al., 1998; Vogt and Marvin, 2006; Hieda and Nam, 2008) and finite element (FE) (Li and Smith, 2015) methods have been reported as modelling approaches. A similar concept is electric resistance tomography (ERT); instead of using induction at radio frequencies, contact electrodes are used along boreholes (Daily and Owen, 1991).

There are many drawbacks to using RIM or ERT in support of a TTE radio deployment. There is no guarantee that a site will have accessible boreholes, or that they will intersect areas of interest. Alternatively, consider modifying the crossborehole geometry by rotating it into a horizontal position, yielding what is effectively a TTE radio configuration albeit with much different equipment. Using RIM in such a configuration could be feasible; however, major equipment modifications would be required. Modifying ERT for such a configuration could be unfeasible; there are safety concerns with placing electrodes into a mine roof, and practicality concerns if too much of the surface above the mine is paved over.

\subsubsection{Transmission tests}

Alternatively, we could attempt to use TTE radio to estimate the conductivity structure of the overburden. Early TTE radio tests, such as those described by Ilsley 
et al. (1928) or Felegy and Coggeshall (1948), were simple feasibility studies. Later experiments, of a more theoretical nature, considered the attenuation of conventional radio waves (Haycock et al., 1949), although controversy ensued whether reception was achieved as a result of the signal travelling down the mine shaft or due to unreasonably high transmitter power, as opposed to reception from a transmitter of typical power (Barret, 1952). The conclusions from these experiments may have been too optimistic, as the tests were done in very resistive media (Pritchett, 1952; McGehee, 1954). Pullen (1953), still operating with conventional radio, explicitly considered the geologic controls on signal attenuation. Various results from the 1950s were revisited both theoretically and experimentally by Dokoupil et al. (1962), who suggested a method to characterize the propagation factor of an overburden by varying the transmitter-receiver separation. Largely theoretical work by Fritsch (1963) demonstrated the feasibility of using TTE transmissions either for communication or extracting information about the conductivity structure of the overburden, although only homogeneous or otherwise electrically simple structures could be modelled at the time.

In the 1970s and 1980s, TTE radio research sponsored by the Bureau of Mines (United States Department of the Interior) was particularly focused on the problem of detecting a miner's signal (Olsen and Farstad, 1973; Geyer et al., 1974; Lagace et al., 1980; Durkin, 1984b), which remains a challenging problem to this day (Sogade et al., 2004; Ayuso et al., 2010; Sheinker et al., 2014). In the ideal case where the Earth is homogeneous, a miner's position can be estimated by locating the null of the radial magnetic field transmitted by a vertical magnetic dipole underground; in addition, at the same position, the vertical magnetic field will reach a peak. A drawback of this approach is that sophisticated modelling is needed to account for complicated conductivity structures that distort the magnetic field (Ayuso et al., 2010; Sheinker 
et al., 2014). From this observation, Sogade et al. (2004) developed a method to map caves by locating the depth and position of a signal transmitted underground; a conductivity distribution was assumed. The transmission tests in Geyer et al. (1974) and Lagace et al. (1980) involved placing a transmitter at the mine level and measuring the signal at the surface. Further investigation (Durkin, 1984a, 1991; Yan et al., 2014) assumed either a halfspace with constant conductivity or a halfspace with a thin conductive sheet at the surface. Tests were conducted at several coal mines in the United States and a regression was fitted to the data from all test locations to estimate apparent conductivity as a function of frequency and depth. This approach presents two major drawbacks: a simplified conductivity structure is assumed, and it eschews site-specific information in lieu of a generalized model for all coal mines in the United States.

There are numerous advantages of using TTE radios versus other methods. First, to encode information, the TTE radio system featured in this study uses a wideband signal that is designed to minimize the effect of powerline noise. Assuming a North American standard, harmonics of $60 \mathrm{~Hz}$ are noise; therefore, the signal is engineered to be stronger at offsets of $30 \mathrm{~Hz}$ from the harmonics and null on the harmonics. Second, analyzing TTE radio data signal will only identify conductivity structures to which the TTE radio is sensitive to; therefore, even if some geological formation was undetected, the resulting conductivity model could still be used to accurately predict signal strength. A conventional EM system could identify such a structure, but it would not add new information to better predict TTE radio signal strength; furthermore, it is possible that another system would miss a conductivity structure to which TTE radio is sensitive. Third, by using a below-ground unit (BGU) and above-ground unit (AGU), the electromagnetic energy is concentrated in overburden between the mine level and surface. This arrangement simplifies analysis, as the TTE 
radios are largely insensitive to the conductivity structure of the subsurface below the mine level. Finally, usng TTE radio equipment confers a logistical advantage as additional systems do not need to be acquired to estimate the conductivity structure of the mine overburden.

\subsection{Theoretical background}

Consider the propagation constant $\gamma$ of an electromagnetic wave of angular frequency $\omega$ in a medium with conductivity $\sigma$, magnetic permeability $\mu$, and electric permittivity $\epsilon$ :

$$
\gamma=\sqrt{j \omega \mu(\sigma+j \omega \epsilon)}
$$

where $j=\sqrt{-1}$. At the frequencies used in TTE radio, $\sigma \gg \epsilon \omega$, thus equation 4.1 simplifies to

$$
\gamma=\sqrt{j \omega \mu \sigma}
$$

or as can be expressed alternatively:

$$
\gamma=(1+j) \sqrt{\frac{\omega \mu \sigma}{2}},
$$

where the real component is the inverse of the skin depth. For most geologic units, the most important electromagnetic rock property is conductivity, although in some geologic settings, the magnetic susceptibility cannot be disregarded. Another factor to consider is the induction number $\mathcal{B}$, which is the ratio of transmitter-receiver separation $r$ and the skin depth $\delta=\sqrt{\frac{2}{\omega \mu \sigma}}$ :

$$
\mathcal{B}=r / \delta
$$


The induction number will vary significantly depending on the conductivity structure of the overburden, the operating frequency, and transmitter-receiver separation.

In our TTE radio system, the transmitter is a vertical magnetic dipole; its vertical magnetic field when placed on the surface is

$$
H_{z}=\frac{M}{2 \pi z^{3}} Q
$$

where $M$ is the magnetic moment of the transmitter; $z$ is the depth; and $Q$ is a propagation factor that is a function of the frequency of the signal $\omega$, the conductivity of ground $\sigma$, the radial distance from the transmitter $\rho$, the radius of transmitter $r$ and the depth $z$ :

$$
\begin{array}{r}
Q=\int_{0}^{\infty} \frac{J_{1}(A x)}{A x / 2} \cdot \frac{x^{3}}{\sqrt{x^{2}+i \zeta^{2}}+x} \cdots \\
\cdots e^{-\left(x^{2}+i \zeta^{2}\right)^{1 / 2}} \cdot J_{0}(D x) d x
\end{array}
$$

where

$$
\begin{gathered}
\zeta=(\mu \omega \sigma)^{1 / 2} z, \\
A=a / z, \\
D=\rho / z
\end{gathered}
$$

and $J_{0}$ and $J_{1}$ are Bessel functions of the first kind (Wait and Spies, 1972). Equation 4.5 assumes that the halfspace is a nonmagnetic material, i.e. $\mu=\mu_{0}$. A similar formula can be constructed for the radial magnetic field $H_{\rho}$ by replacing the propagation factor. By inspection, as the propagation factor $Q$ decreases, less signal can be received.

Plots of the vertical magnetic field $\left(H_{z}\right)$ versus conductivity, frequency, and radial 
distance are presented in Figures 4.1-4.3. When the transmitter and receiver are aligned coaxially $(\rho=0), H_{z}$ decreases monotonically if frequency or conductivity increases - as the skin depth decreases, we intuitively expect less signal. However, if $\rho>0$, we start to observe non-intuitive behaviour. Consider Figures 4.1 and 4.2, for a depth of $20 \mathrm{~m}$ and a radial distance of $50 \mathrm{~m}$. Attenuation can be reduced twofold (i.e. $6 \mathrm{~dB}$ gain) by choosing an appropriate carrier frequency provided that the conductivity of the medium is known. Based on experience with deploying TTE radios, the signal quality will decrease over a range of 5-6 $\mathrm{dB}$ as the bit error rate (i.e. amount of corrupted information) increases, until the signal is indistinguishable from the noise. In some situations, a small change in conductivity will cause a large change in the signal; for example, in Figure 4.1, for a carrier frequency of 8000 $\mathrm{Hz}$, attenuation is increased threefold (10 dB loss) when the conductivity of the homogeneous halfspace increases from $0.2 \mathrm{~S} / \mathrm{m}$ to $0.4 \mathrm{~S} / \mathrm{m}$. As seen in Figure 4.3, while the signal will generally be more attenuated away from the transmitter, some areas of much lower signal, termed dead zones, do not exist when the conductivity increases. These dead zones can be explained by the change in sign of the vertical magnetic field.

As seen in Figures 4.1-4.3, there are optimal frequencies for which significantly more signal can penetrate the ground; the rule of thumb that lower frequencies will always deliver a stronger signal does not always hold. Knowing the conductivity distribution of the subsurface would allow a crew to predict the location of any dead zones, which would be avoided by either appropriately positioning the transmitterreceiver pair or changing the carrier frequency. Knowing the conductivity distribution of the subsurface would allow to predict the maximum range (both in terms of depth and radial distance) of operability of the TTE radio system. 


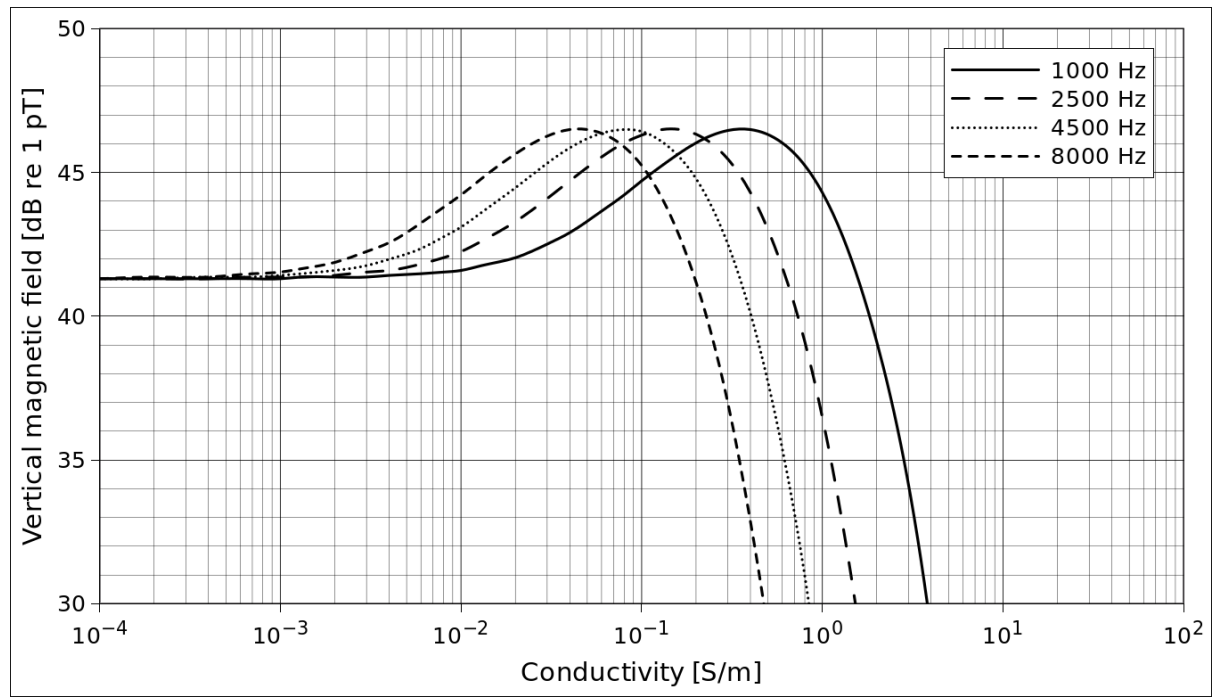

Figure 4.1: Vertical magnetic field in a homogeneous halfspace versus conductivity for four typical TTE radio carrier frequencies. A circular transmitter of radius $10 \mathrm{~m}$ and current $1 \mathrm{~A}$ is placed on the air-ground interface; the receiver is located at depth of $20 \mathrm{~m}$ and a radial distance of $50 \mathrm{~m}$.

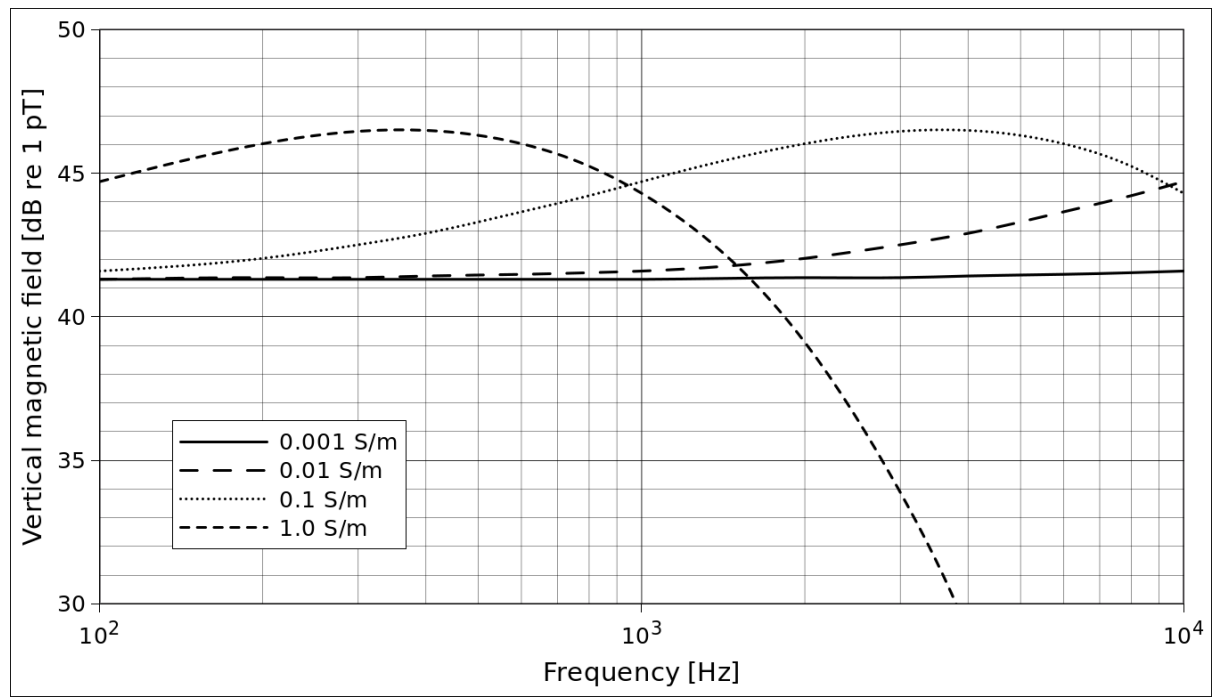

Figure 4.2: Vertical magnetic field in a homogeneous halfspace versus carrier frequency for four plausible conductivities of a mine overburden. A circular transmitter of radius $10 \mathrm{~m}$ and current $1 \mathrm{~A}$ is placed on the air-ground interface; the receiver is located at depth of $20 \mathrm{~m}$ and a radial distance of $50 \mathrm{~m}$. 


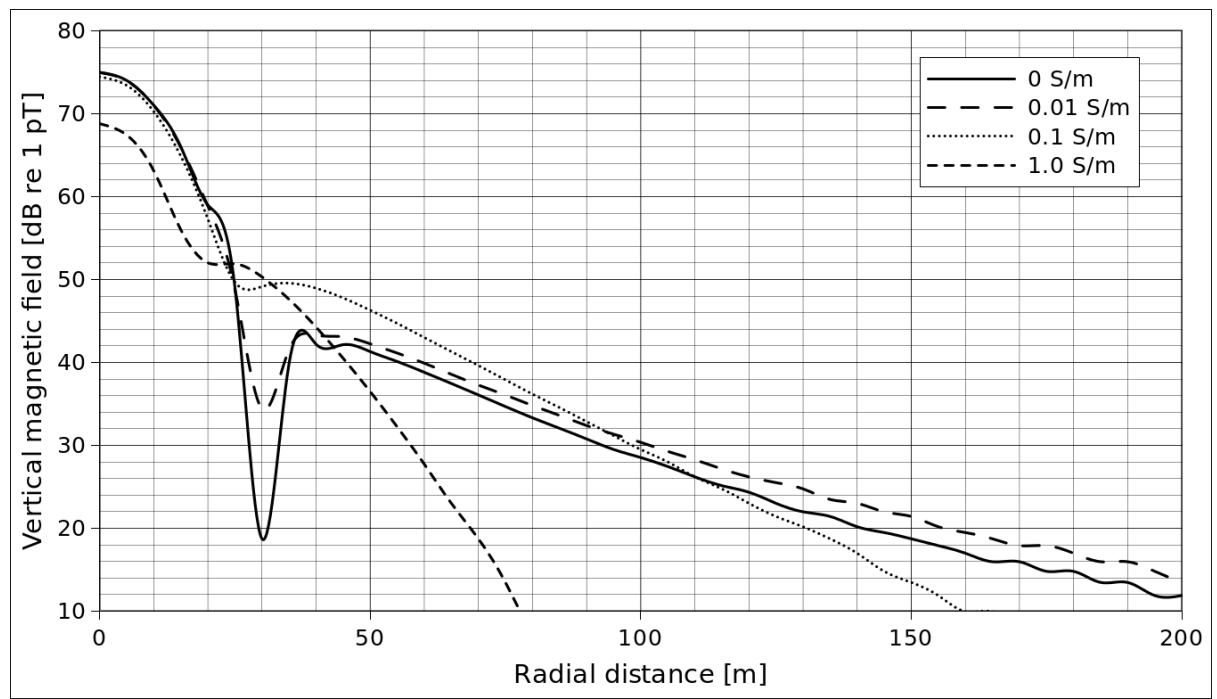

Figure 4.3: Vertical magnetic field in a homogeneous halfspace versus radial distance at a frequency of $2500 \mathrm{~Hz}$ for air and three plausible conductivities of a mine overburden. A circular transmitter of radius $10 \mathrm{~m}$ and current $1 \mathrm{~A}$ is placed on the air-ground interface; the receiver is located at depth of $20 \mathrm{~m}$.

\subsection{Proposed approach}

Following Durkin (1984a, 1991) and Yan et al. (2014), apparent conductivity for a homogeneous halfspace model can be obtained by rearranging equation 4.5 to solve for $Q$. Alternatively to a homogeneous halfspace model, a thin sheet of known conductance at the surface can be added. (Durkin, 1997; Yan et al., 2014). A final sophistication was proposed by Yan et al. (2014) by finding an apparent conductivity for a homogeneous halfspace based on a propagation factor determined for the thin sheet model. We propose to improve upon this work by using the finite-difference time-domain (FDTD) code developed for modelling TTE radio transmissions in Ralchenko et al. (2015) to fit a conductivity model.

A proof-of-concept study was done to invert the apparent conductivity from $Q$ values, using synthetic data. This approach is based solely on the change in amplitude of the signal as it propagates through the Earth, in contrast to conventional 
electromagnetic methods in exploration geophysics, which need a phase reference to decompose the total signal into its primary and secondary components. We consider the case of a horizontal transmitter of radius $10 \mathrm{~m}$ placed on the air-ground interface. The receiver is located $18 \mathrm{~m}$ coaxially below the transmitter. The ground is a homogeneous halfspace of conductivity $1 \mathrm{~S} / \mathrm{m}$. We model a current of $1 \mathrm{~A}$ in the transmitter loop. The model excitation waveform used is a cosine-modulated Gaussian function with a central frequency of $4500 \mathrm{~Hz}$ and a bandwidth of $4000 \mathrm{~Hz}$. It is defined in the time domain by

$$
f(t)=\cos \left(\omega_{c} t\right) \cdot e^{-\left(\frac{t^{2}}{\tau}\right)},
$$

where $\tau$, a parameter used to describe the width of a Gaussian pulse, is dependent on a central frequency $\omega_{c}$ and the bandwidth $\Delta f$ :

$$
\tau=\frac{2 \sqrt{2.3}}{\pi \Delta f}
$$

In the frequency domain, this function is bell-shaped, with a peak at the central frequency and its width equal to the bandwidth:

$$
F(\omega)=\frac{\tau \sqrt{\pi}}{2}\left(e^{-\left(\frac{\tau\left(\omega-\omega_{c}\right)}{2}\right)^{2}}+e^{-\left(\frac{\tau\left(\omega+\omega_{c}\right)}{2}\right)^{2}}\right) .
$$

FDTD simulations were performed for the aforementioned scenario. For comparison, an equivalent scenario was modelled where the conductivity of the halfspace was replaced with that of air. As expected, higher-frequency components are more attenuated in a conductive medium (Figure 4.4). The amplitudes for frequencies between $1000 \mathrm{~Hz}$ and $8000 \mathrm{~Hz}$ were inverted to yield the original conductivity of $1 \mathrm{~S} / \mathrm{m}$. 


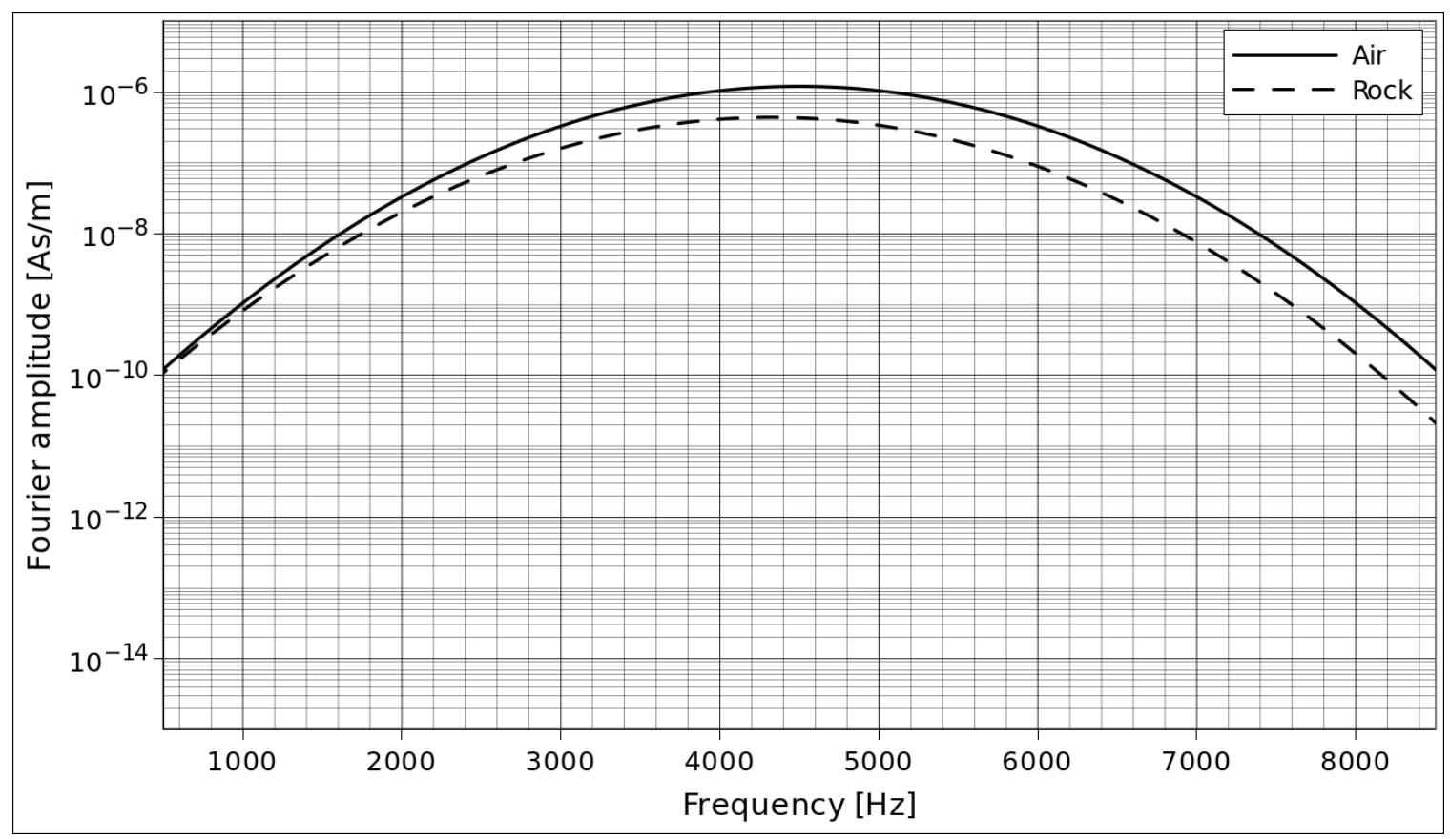

Figure 4.4: Fourier spectra of synthetic data generated using the FDTD method. The attenuation in the rock increases with higher frequency.

\subsection{Field survey}

A field survey was conducted at the Safety Research Coal Mine at the National Institute for Occupational Safety and Health (NIOSH), in Bruceton, Pennsylvania, United States, near Pittsburgh. Originally hand dug as adits into the Pittsburgh coal seam at the turn of the 20th century, this mine, as well as the adjoining Experimental Coal Mine, are room-and-pillar type mines, and have been used for communications experiments as early as the 1920s (Ilsley et al., 1928). We transmitted signal using a circular antenna of radius $10 \mathrm{~m}$ from two surface locations, and made receiver measurements at 16 different locations within the mine (Figure 4.5a); in total, 24 measurements were made (subsurface locations 1-8 were not surveyed with the transmitter Tx2). Figure $4.5 \mathrm{~b}$ is a $3-\mathrm{D}$ representation of the mine, combining a digital elevation model (DEM), and mine- and surface-level features. 


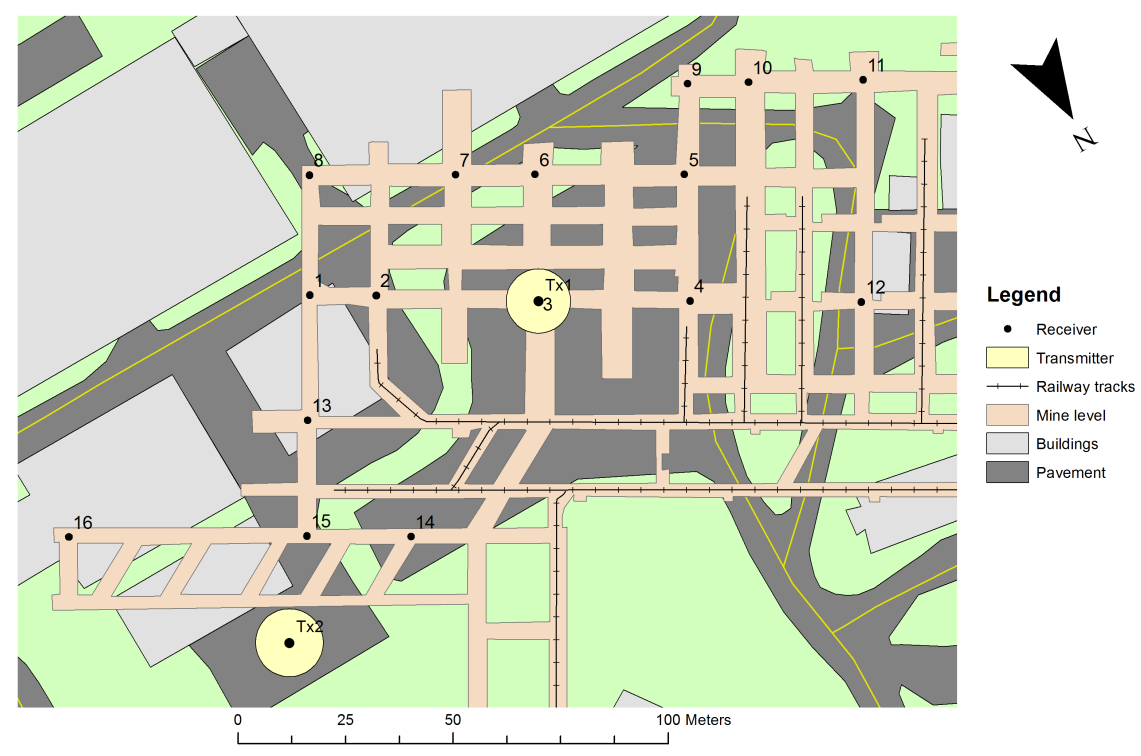

(a)

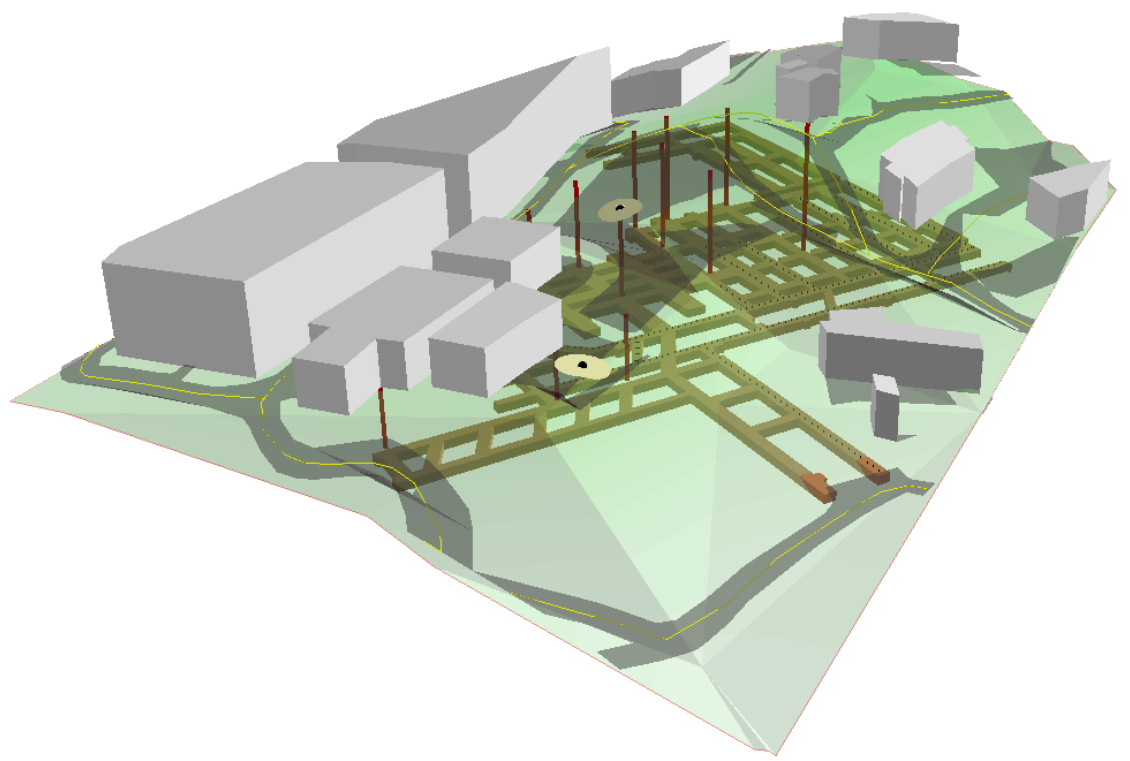

(b)

Figure 4.5: Map (a) and 3D view (b) of the Safety Research Coal Mine. The 3D view has a vertical exaggeration of 1.5 , and an elevation range on the order of $30 \mathrm{~m}$. 
A wideband TTE radio signal was transmitted. The waveform is shown in Figure 4.6. This type of signal waveform, termed interharmonic modulation (IHM), is designed such that the carriers, which are the individual peaks in the Fourier spectrum that encode information, are spaced between power line harmonics. Since our survey site was located in the United States, the power line AC frequency is $60 \mathrm{~Hz}$. The TTE radio signal was therefore offset by $30 \mathrm{~Hz}$ from the power line noise. Such a waveform can be described with regards to a central frequency about which 32 carriers are spaced $60 \mathrm{~Hz}$ apart (or $50 \mathrm{~Hz}$ if appropriate given power line frequency), for a bandwidth of $1920 \mathrm{~Hz}$. For our survey, we used central frequencies of $1500 \mathrm{~Hz}$, $3500 \mathrm{~Hz}, 5500 \mathrm{~Hz}$, and $7500 \mathrm{~Hz}$. This approach builds on similar experiments in the literature (Geyer et al., 1974; Lagace et al., 1980), which used four single-frequency signals (specifically, $630 \mathrm{~Hz}, 1050 \mathrm{~Hz}, 1950 \mathrm{~Hz}$, and $3030 \mathrm{~Hz}$ ). Additionally, those experiments used only one transmitter and receiver location each whereas we used two transmitter locations and 16 receiver locations.

In their raw form, the measured magnetic field signals contained many hundreds of repeating TTE radio data waveforms. Superimposed on top of the raw signals were $60 \mathrm{~Hz}$ power line harmonics, plus any transient natural electromagnetic events (e.g. sferics). Timing recovery was performed using autocorrelation on a coarsely filtered signal time series, in order to identify the start times of all TTE radio waveforms. Next, the TTE radio waveforms were extracted individually. Subsample interpolation was used for increased accuracy. Finally, all individual waveforms were stacked using simple boxcar coefficients (i.e. averaged together) to arrive at the final TTE radio waveform suitable for further interpretation. Following windowing using a BlackmanHarris function, a Fourier transform was performed on the final time-domain waveform to extract the amplitude of each carrier frequency. The transmitter current waveform was recorded separately; each recording, however, was clean enough not to 


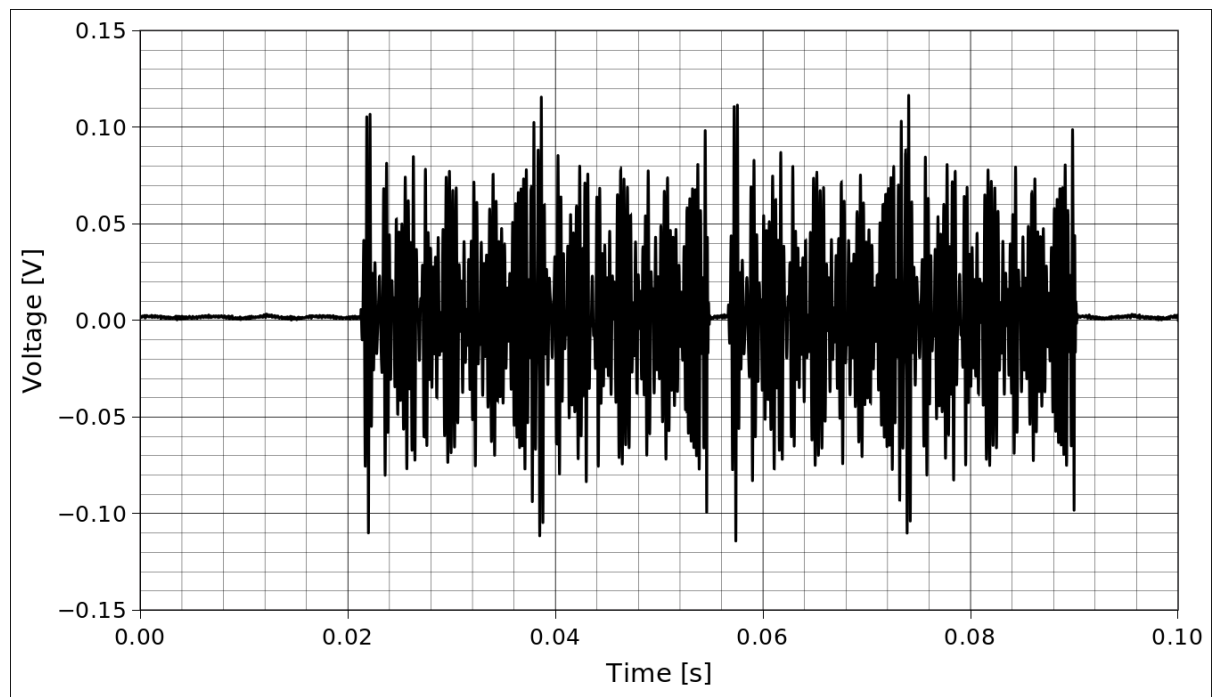

(a)

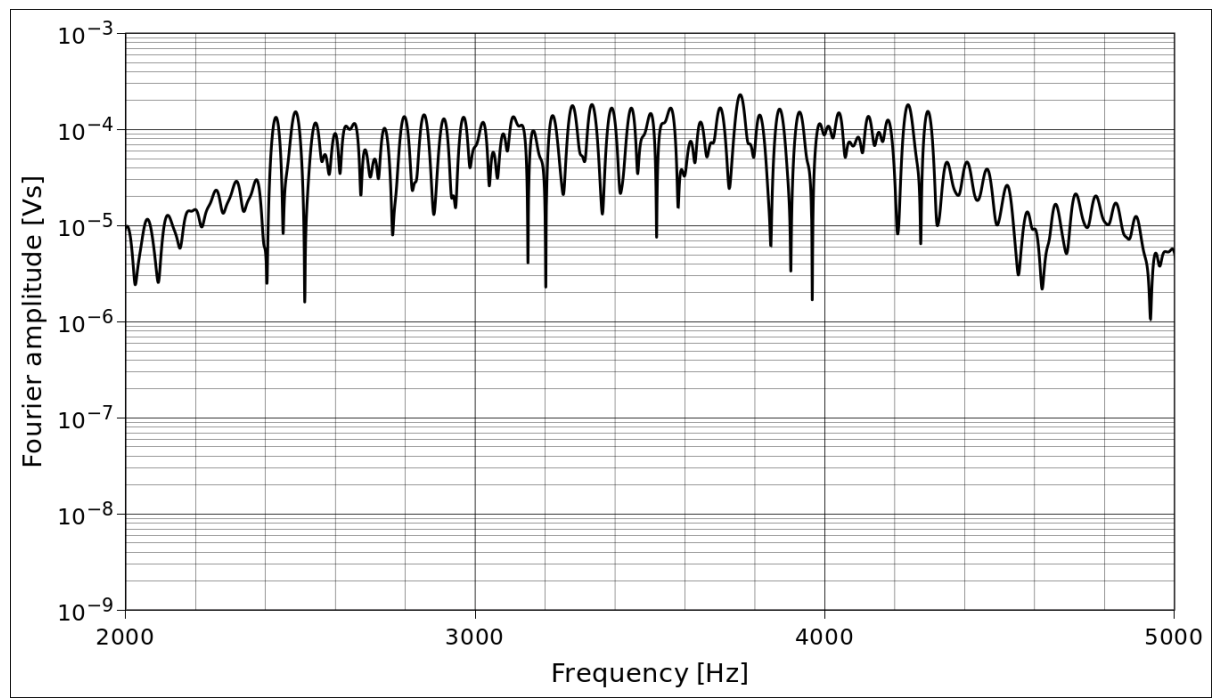

(b)

Figure 4.6: TTE radio interharmonic modulation (IHM) waveform in time domain (a) and frequency domain (b). In (a), a signal encoding a single symbol is shown. This symbol is continuously transmitted in these tests. In the Fourier spectrum (b), the peaks are offset by $30 \mathrm{~Hz}$ from the $60 \mathrm{~Hz}$ power line harmonics, which permits a TTE radio link in the presence of high ambient noise. Note that the timeseries has been stacked and filtered. 
require further processing other than windowing and a Fourier transform to permit comparison with the underground recordings.

\subsection{Field data processing and analysis}

\subsubsection{Data quality and uncertainty}

Of the 24 recordings made, nine had usable data. Eight stations had an unrealistically high magnetic field that cannot be explained by any overburden conductivity distribution or reasonable acquisition geometry. The likely cause for the high magnetic field is the presence of elongated metallic conductors, such as railways, within the mine. In other surveys, TTE signals have been observed to be amplified in the vicinity of long conductors via the poorly understood phenomenon of parasitic coupling (Ralchenko et al., 2016). A similar phenomenon is the coupling of geophysical signals to elongated wires (Szarka, 1988; McKenna et al., 2013). While it is known where the railways are, there are vertical conductors, such as borehole casings, whose position and quantity is unclear, which makes accounting for such objects during data analysis impractical. Finally, seven did not have data suitable for processing, as it was either clipped (for stations very close to the transmitter) or had a poor signal-to-noise ratio (for stations very far from the transmitter).

Durkin (1991) determined that the uncertainty in $Q$ is on the order of $\pm 20 \%$, largely based on an uncertainty of $\pm 10 \%$ in the loop area. The experimental setup described by Durkin (1991) involved placing the transmitter wire underground around coal pillars, and estimating its area based on mine maps, whereas our transmitter was on the surface, which made it easier to constrain the transmitter geometry. TTE radio signal strength is a logarithmic quantity that is best quoted in decibels. An uncertainty in signal of $\pm 20 \%$, or $\pm 1.6 \mathrm{~dB}$, is small, compared to variations of tens 
of decibels of signal which occur when changing position, frequency, or conductivity. In contrast, if a circular loop of radius $10 \mathrm{~m}$ is distorted into a $12 \mathrm{~m}$ x $8 \mathrm{~m}$ ellipse (an unrealistically high error for deployment on a clear parking lot), the resulting error in the loop area is 4\%. Based on the assumptions of Durkin (1991), the corresponding overall error in $Q$ is $8 \%$, or $0.6 \mathrm{~dB}$. Geometry is thus a small source of error in our experiment, and the largest source of error is parasitic coupling.

\subsubsection{Homogeneous halfspace model}

The propagation factor $Q$ is obtained as a function of frequency from on equation 4.6:

$$
Q(f)=\frac{H_{z}(f)}{N I(f)} \frac{2 \pi z^{3}}{\mathcal{A}}
$$

where $I(f)$ is a frequency-dependent current, $N$ is the number of turns in the loop, and $\mathcal{A}$ is the loop area. The purpose of obtaining $Q$ is three-fold: first, $Q$ is independent of the transmitter current at each frequency; second, an apparent conductivity can be obtained based on a homogeneous halfspace model; and third, it is a preliminary step for further analysis.

We consider the example for the transmitter-receiver pair of transmitter Tx1 and receiver 9. Figure 4.7 shows $Q$ as a function of frequency. The conversion of $Q$ to conductivity is shown graphically in Figure 4.8; $Q$ based on four frequencies $(1000 \mathrm{~Hz}$, $2500 \mathrm{~Hz}, 4500 \mathrm{~Hz}$, and $8000 \mathrm{~Hz}$ ) representative of the $Q$-f graph (Figure 4.7) has been plotted versus conductivity. A $20 \%$ uncertainty in $Q$ is assumed, which yields a corresponding uncertainty in apparent conductivity. As shown in Figures 4.1-4.3, a single propagation factor can result from multiple conductivities. For this case, we choose the upper estimate for apparent conductivity as the lower estimates would start to introduce a sharp discontinuity in the apparent conductivity-frequency relationship, 


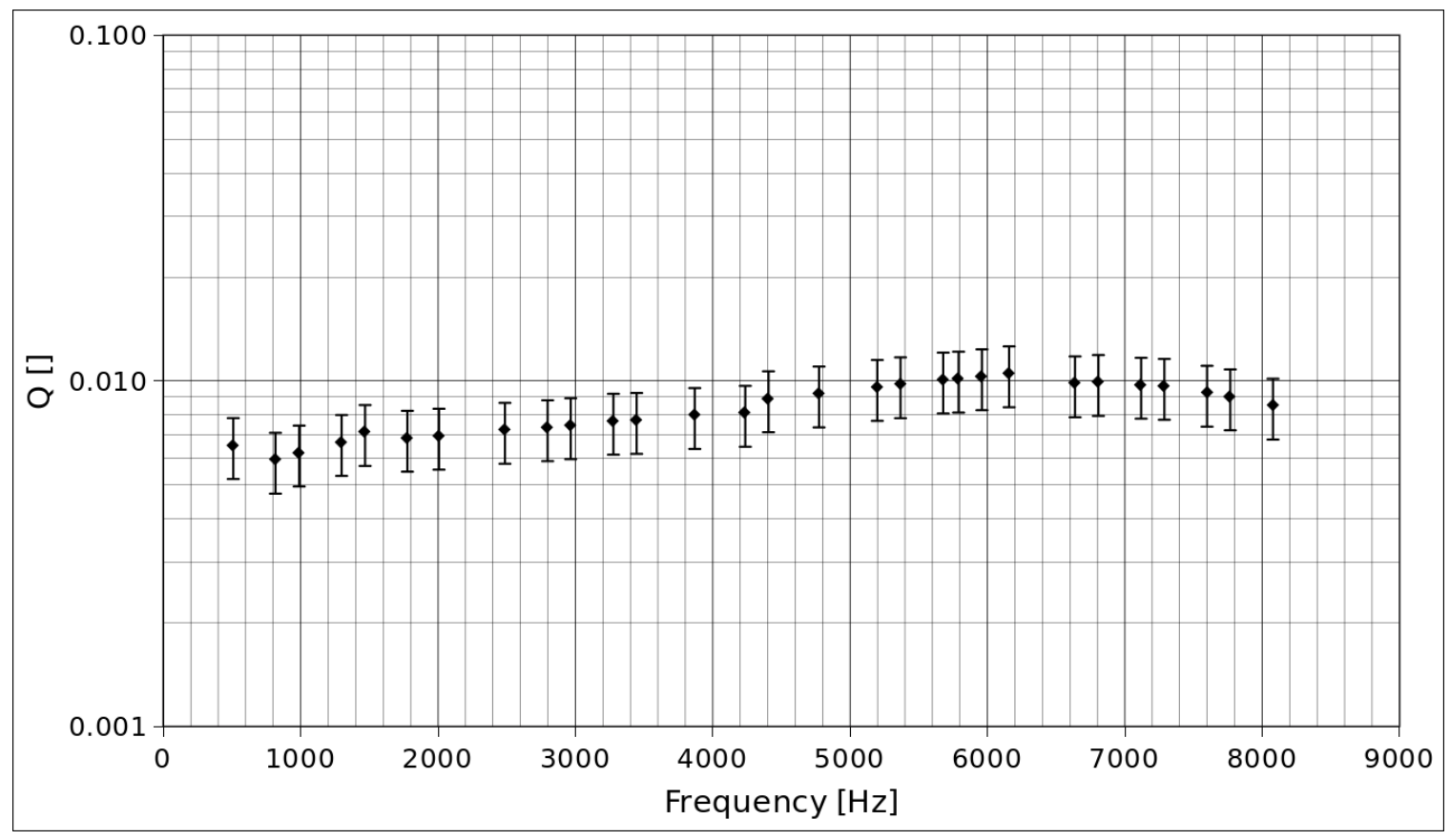

Figure 4.7: Propagation factor $Q$ versus frequency for receiver 9 and transmitter Tx1.

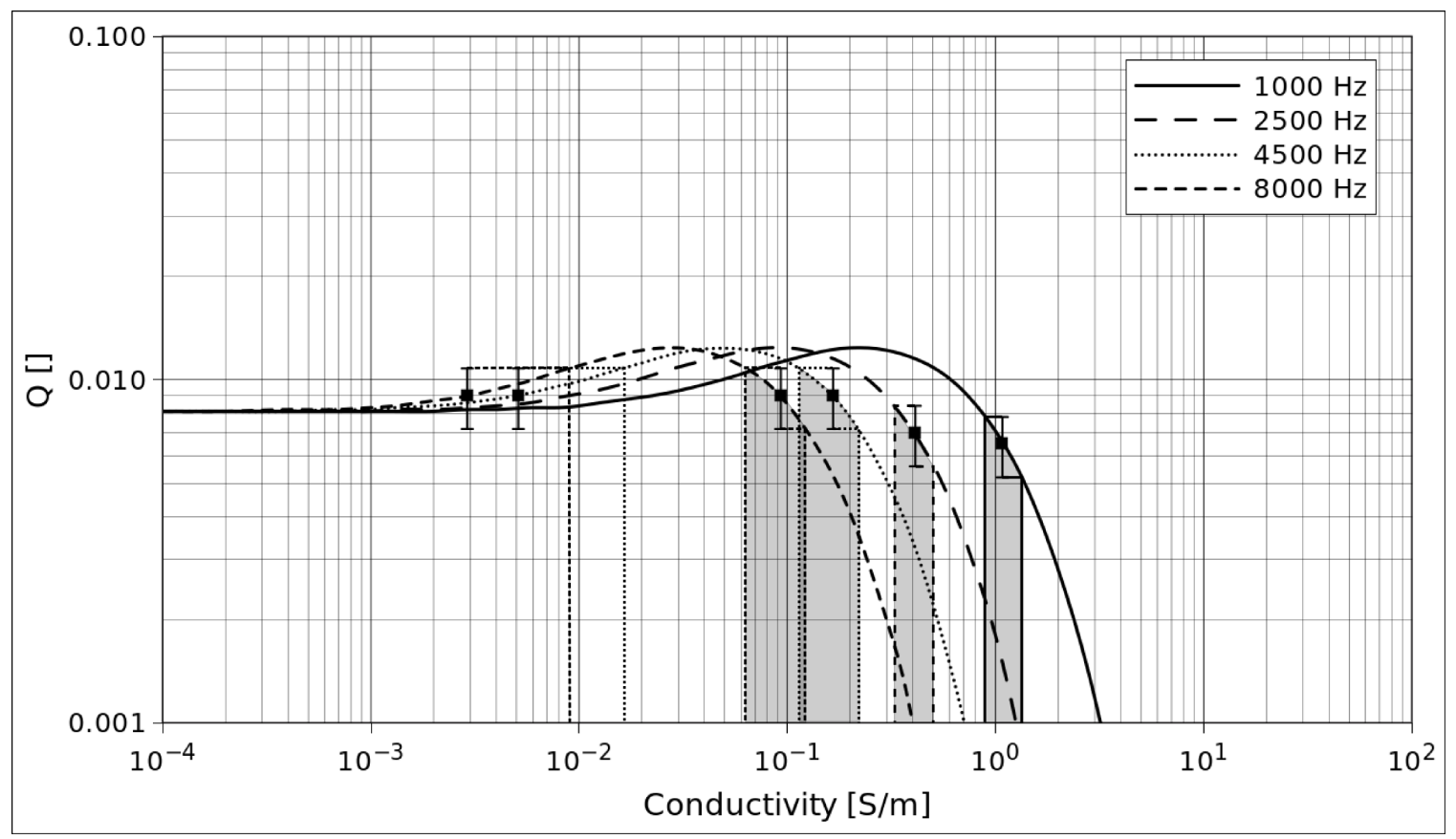

Figure 4.8: Propagation factor $Q$ at location 9 and transmitter Tx1 for four representative frequencies. The range of possible conductivities corresponding to a $20 \%$ uncertainty in $Q$ is shaded in grey. 
which is a less plausible scenario. Finally, note that it becomes difficult to resolve low conductivities at low frequencies - at this propagation factor and associated uncertainty, any apparent conductivity less than $0.01 \mathrm{~S} / \mathrm{m}$ will fit the data at $4500 \mathrm{~Hz}$ and $8000 \mathrm{~Hz}$. The final result is given in Figure 4.9.

The most important observation from Figure 4.9 is the frequency dependence of the apparent conductivity. The frequency dependence is indicative of a nonhomogeneous subsurface, as the model assumed a homogeneous halfspace; the discrepancy is apparent at the test site from the presence of a clayey soil overlying several sedimentary formations. A result of $1 \mathrm{~S} / \mathrm{m}$ is an exceedingly conductive geologic medium - even at a coal mine. Were the overburden uniformly of that conductivity, much stronger attenuation at higher frequencies would be observed. For example, comparing a homogeneous halfspace of $1 \mathrm{~S} / \mathrm{m}$ and $0.03 \mathrm{~S} / \mathrm{m}$, for a transmitter of radius $10 \mathrm{~m}$, operating at $8 \mathrm{kHz}$ with a vertical transmitter-receiver separation of $12 \mathrm{~m}$, the signal in the more conductive medium will be $8 \mathrm{~dB}$ lower when the horizontal transmitter-receiver separation is $0 \mathrm{~m}$, and $34 \mathrm{~dB}$ lower when the horizontal transmitter-receiver separation is $50 \mathrm{~m}$. Strong attenuation at higher frequencies was not observed in any of the nine recordings yielding usable data. Previous authors who performed a transmission test for several frequencies and fitted a homogeneous halfspace model to the data also report a marked decrease of conductivity with frequency at coal mines in the United States (Durkin, 1984a, 1991; Yan et al., 2014). A final note: while rock is dispersive material whose conductivity increases with frequency, such an effect is minimal over one decade of frequency.

\subsubsection{Layered Earth model}

During the visit to the field site, it was readily apparent that the overburden geology could not be approximated as a homogeneous halfspace. Based on these observations 


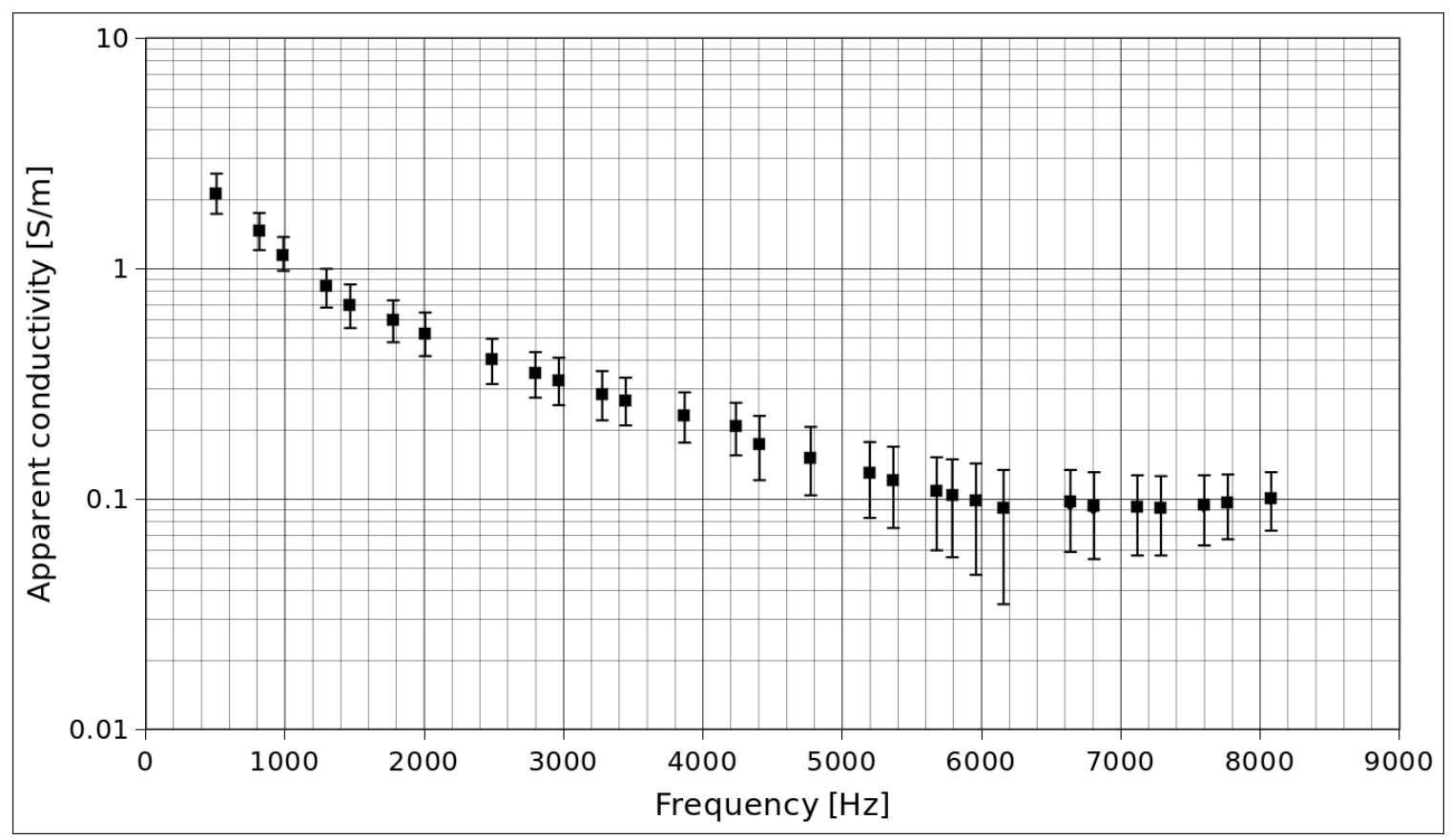

Figure 4.9: Apparent conductivity versus frequency for receiver 9 and transmitter Tx1.

and a literature survey (Ilsley et al., 1928), the mine overburden is composed of a soil layer that overlies several sedimentary units consisting of sandstones and shales. Therefore, it becomes necessary to use a more sophisticated conductivity model to adequately describe the mine overburden.

The algorithm used to fit a layered Earth conductivity model is illustrated in Figure 4.10 and is explained as follows. The geological model used was a four-layer model, consisting of a soil layer, two bounded layers, and a halfspace. Additionally, the considerable topography at the test location was accounted for based on a digital elevation model (DEM). Initial conductivity values were assigned to this model based on constraints obtained from having previously fitted apparent conductivities based on a homogeneous halfspace model and from the lithologies. Specifically, possible conductivities for any layers were constrained to be between $0.001 \mathrm{~S} / \mathrm{m}$ and $1.0 \mathrm{~S} / \mathrm{m}$. The lower limit is implied by the local geology, but it is also effectively a consequence 


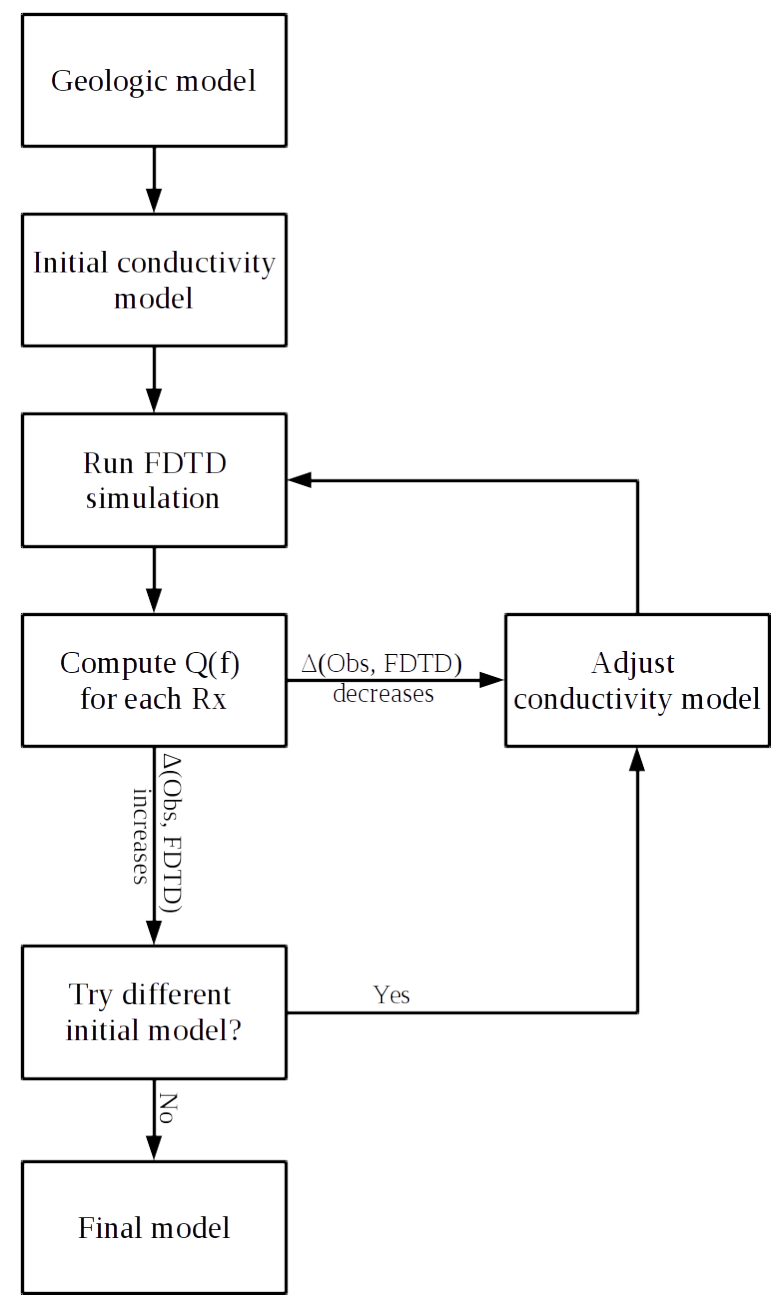

Figure 4.10: Flowchart for fitting a four-layer conductivity model 
of the inability of the method to resolve conductivities below $0.001 \mathrm{~S} / \mathrm{m}$, as the contribution of the attenuation due to the presence of a conductive medium at some distance away from the transmitter would not be distinguishable against the geometric attenuation. The upper limit is dictated by the soil conditions and the apparent conductivities based on a homogeneous halfspace model. We further assume that magnetic permeability $\mu$ is equal to that of free space $\left(\mu_{0}=4 \pi \times 10^{-7} \mathrm{H} / \mathrm{m}\right)$, and that given the frequencies used and the probable conductivities, we cannot distinguish changes in relative electric permittivity $\epsilon_{r}$.

An FDTD-based forward modeller was used to fit a conductivity model to the observed data. There are several advantages of using FDTD for this task. First, as it is a time-domain method, all frequencies of interest can be run simultaneously. Furthermore, all receivers associated with one transmitter location can be modelled simultaneously; note that transmitters and receivers are separated from each other in three-dimensional space. Finally, using FDTD allows to account for topographical variation. The original data were vertical magnetic field intensities for several frequencies over a range of 500-8500 Hz. A complication of using a wideband signal is that the transmitted current is not necessarily the same at each frequency of interest. To avoid this problem, the measured vertical magnetic field intensity was converted to the propagation factor $Q$. To streamline the interpretation process, four frequencies of interest $(1000 \mathrm{~Hz}, 2500 \mathrm{~Hz}, 4500 \mathrm{~Hz}$, and $8000 \mathrm{~Hz})$, which are sufficient to reconstruct the $Q-f$ profile, are used in further interpretation. Fitting is accomplished by minimizing the average of the absolute differences between the 23 values for modelled $Q$ and observed $Q$ (Table 4.1). The difference in $Q$ between modelled and observed values is equivalent to the difference between modelled and observed TTE radio signal strength.

Plausible conductivity values are manually fitted for each of the layers based on the 
Table 4.1: Deviation of observed and calculated $Q$ in decibels for the model shown in Figure 4.11.

\begin{tabular}{c|cccccccc|c} 
& \multicolumn{7}{c|}{ Receiver location } & Transmitter Tx2 \\
\cline { 2 - 10 } & \multicolumn{7}{c}{ Transmitter Tx1 } & 13 \\
\cline { 2 - 9 } & 9 & 8 & 12 & 11 & 6 & 5 & 4 & 1 & -3.0 \\
\hline $1000 \mathrm{~Hz}$ & 2.4 & 4.8 & 4.4 & -2.7 & -6.4 & -2.6 & -5.0 & 5.4 & -1.6 \\
$2500 \mathrm{~Hz}$ & 3.0 & 6.8 & 2.5 & -2.5 & -5.6 & -1.0 & -2.3 & - & -2.3 \\
$4500 \mathrm{~Hz}$ & 1.8 & 6.6 & 1.0 & - & - & 0.4 & 0.1 & - & - \\
$8000 \mathrm{~Hz}$ & 2.1 & 0.8 & - & - & - & - & - & 0.3 & -
\end{tabular}

aforementioned constraints. By trial and error, a conductivity model was chosen that had an optimal fit relative to other solutions, and was plausible (e.g. conductivities fit within the constraints, the magnitudes were plausible for the known geology, or relative conductivities between the layers were plausible, i.e. soil is more conductive than rock). For this final model, making small adjustments to the conductivities did not improve the fit. Quantitatively, this criterion means that average of the absolute differences is minimized within $0.1 \mathrm{~dB}$. This limit is motivated by the fact that the signal strength as measured by the TTE radios used is within $0.1 \mathrm{~dB}$; therefore, minimizing beyond $0.1 \mathrm{~dB}$ is not significant. There are two corollaries from this choice.

The true conductivities fitted for the four-layer model are shown in Figure 4.11. The difference between observed and modelled signal strength is expressed in decibels in Table 4.1. When the signal fades out, the bit error rate will gradually increase over a range of 5-6 dB until synchronization between transmitter and receiver (reception of a reference signal) is lost. The average absolute deviation of $3.1 \mathrm{~dB}$ in Table 4.1 is below this threshold. The model of Figure 4.11 is therefore accurate enough to be used to optimize the setup of a TTE radio system at the mine site. As an additional check of its accuracy, the same model was used for receiver 9 but transmitter Tx2. 


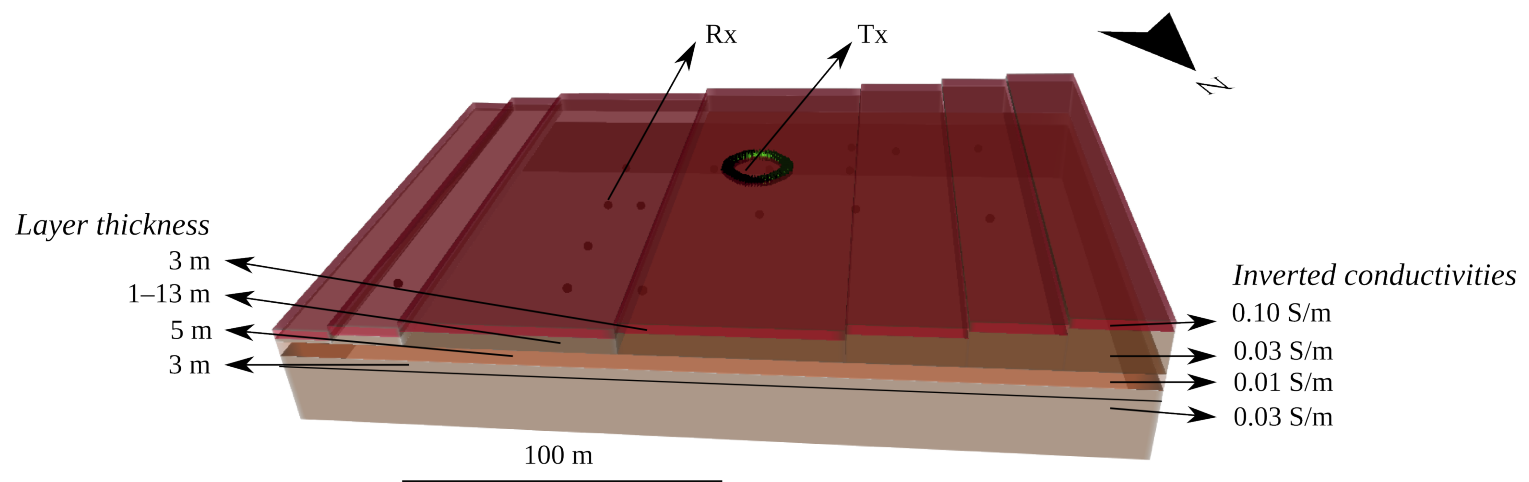

Figure 4.11: Four-layer model with fitted conductivities for each layer. The staircase pattern is a consequence of discretizing the topography using finite differences. No vertical exaggeration.

The average absolute deviation for the three additional data points is $2.3 \mathrm{~dB}$, which suggests a good fit. Another visual check is shown in Figure 4.12, which shows good agreement between the observed and calculated curves for propagation factor or apparent conductivity versus frequency.

There was a large range in apparent conductivities in the homogeneous halfspace model that is not seen in the fitted model. The first cause is the presence of multiple layers of different conductivity. The second cause is a combination of the uncertainty in the propagation factor, often having two possible conductivities returning the same propagation factor, and the difficulty in resolving lower-conductivity features at low frequencies. At low frequencies in particular, small negative errors in observed attenuation sharply skew the apparent conductivity to high values. Models where all layers have high conductivities (at least $0.1 \mathrm{~S} / \mathrm{m}$ ) were clearly incorrect. However, a realistic model was constrained as data at several locations and frequencies was available.

The derived conductivity model, however, has several limitations, such as its inability to account for parasitic coupling with the railway tracks. Another complication is the potential presence of lateral inhomogeneities, which may explain differences in 


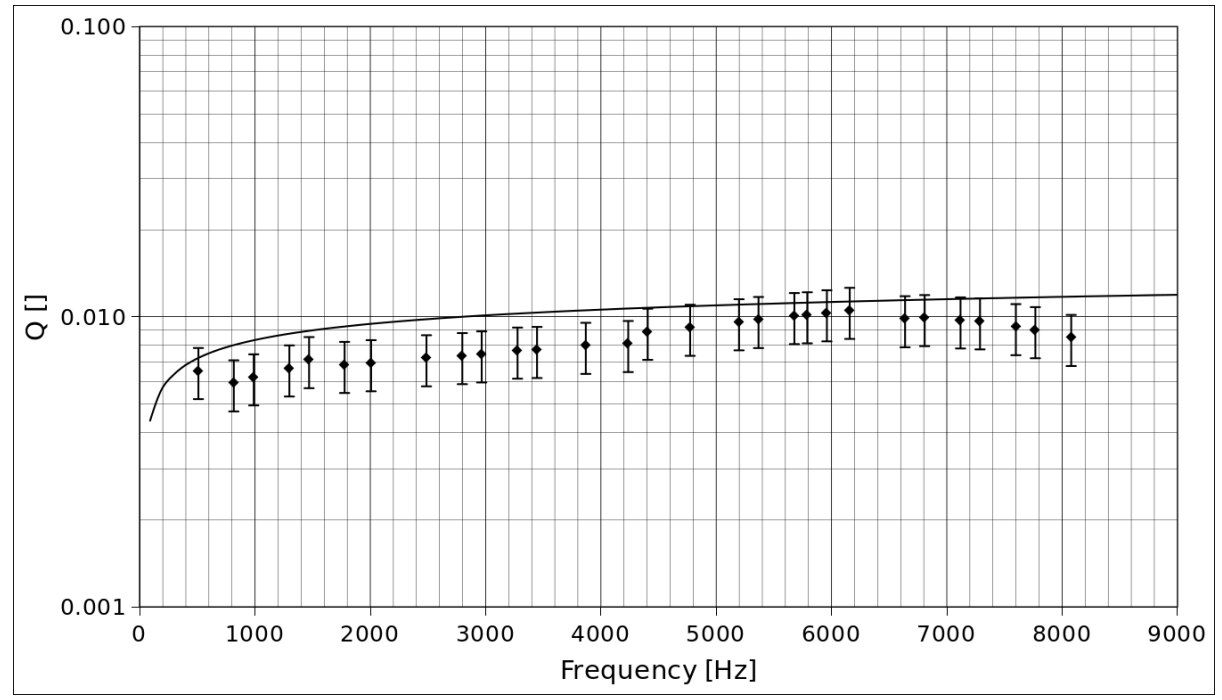

(a)

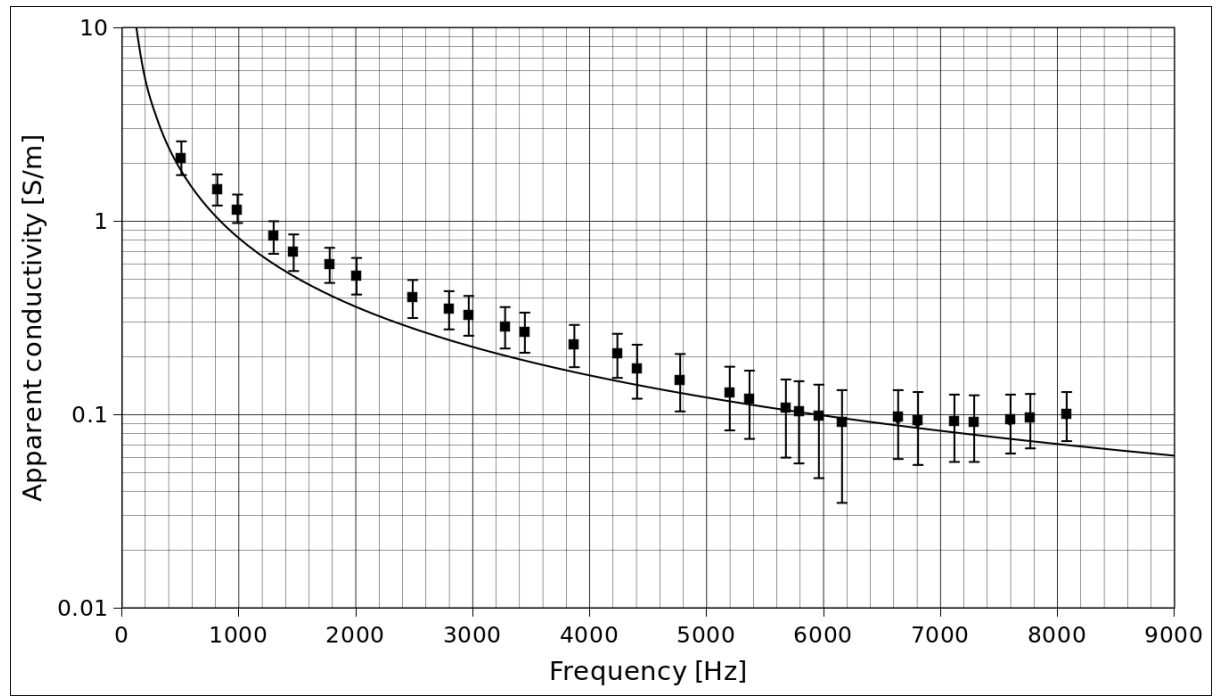

(b)

Figure 4.12: Calculated (solid line) and observed data (square symbols) after fitting a conductivity model to the data presented in Figure 4.7 and 4.9. 
the frequency-dependent attenuation between various locations. While the shale making up the bulk of the mine overburden is undoubtedly anisotropic, this effect should be minimal as a horizontal transmitter loop was used.

\subsection{Summary and conclusions}

A method has been developed to estimate site-specific conductivity data in support of a TTE radio deployment. Although the initial goal of this research was to improve TTE radio communications, a method was demonstrated by which conductivity information can be obtained for a mine overburden.

A series of wideband TTE radio signals were transmitted from the surface and recorded at underground receivers at several locations. The large frequency range $(500-8500 \mathrm{~Hz})$, as well as the variety of transmitter-receiver locations allowed us to obtain a plausible model for the conductivity structure of the mine overburden. This model was fitted by minimizing the absolute difference between the recorded and calculated attenuation coefficients $Q$. The overall discrepancy between observed and modeled data of $3.1 \mathrm{~dB}$ is sufficient to select TTE radio settings, such as central frequency, or to determine maximum signal range or ideal transmitter-receiver locations - for the digital radios used in the experiment, the ability to receive signal fades out over a range of 5-6 dB.

Nevertheless, there are complications to this approach, namely the impact of the signal coupling to elongated metallic objects, such as railway tracks common in coal mines. While other factors contribute to the uncertainty, such as geometry, they are minimal compared to the signal amplification thought to be occurring due the presence of the railway tracks (Ralchenko et al., 2016). Interpretation of the results could be improved through the better understanding of the coupling effects, as well 
as the ability to integrate such objects into numerical models.

An estimate of a site-specific conductivity distribution is a powerful piece of information for optimizing TTE radio location and operating frequency. In a mine emergency, communication would be attempted between a BGU and an AGU, each with their own transmitter and receiver. The position of the BGU is usually constrained (e.g. at a refuge chamber), although if miners could carry a portable terminal, a range of positions would be needed. Given a site-specific conductivity distribution, it is first possible to estimate the maximum range of the TTE radios, which will narrow down what locations are optimal for setting up an AGU, or, in the case of several AGUs, which one to use. Finally, an optimal operating frequency can be chosen. In most cases, lowering the frequency will have the effect of improving signal range; however, there exist locations where increasing the operating frequency will increase the signal. Changes from an initial frequency would be accommodated by tuning the BGU to an operating frequency chosen from a discrete set. Such an insight could only be made if an estimate of the site-specific conductivity distribution existed.

\subsection{Acknowledgments}

We wish to thank Randy Enkin (Natural Resources Canada) and Michel Chouteau (École Polytechnique, Montréal) for the enlightening discussions that motivated this work. We further wish to thank the National Institute for Occupational Safety and Health (NIOSH) of the United States for providing access to the Safety Research Coal Mine, as well as Nicholas Damiano and Bruce Whisner for their assistance during our visit. This research project is funded by the Natural Sciences and Engineering Research Council of Canada (NSERC) and Vital Alert Communications, Inc., through an Industrial Postgraduate Scholarship to M. Ralchenko. 


\section{Chapter 5}

\section{Coupling of very low frequency through-the-Earth radio signals to elongated conductors}

\subsection{Abstract}

Through-the-Earth radio signals (400-9000 Hz) have been observed to couple to elongated conductors present near the transmitter. This phenomenon, which greatly increases signal range, is poorly understood. Experimental and numerical data are combined to better understand the potential of such a communication link. A finitedifference time-domain (FDTD) code is used with a thin wire approximation to model the elongated conductors, which include railway tracks and an elevator shaft. The coupling effect, attributed to currents induced in the elongated conductor, is characterized based on measurements of the three magnetic field components in the vicinity of the conductors. The ability of FDTD to handle arbitrary material geometries makes it an effective tool not only for reconciling theoretical and experimental results, but also for predicting the viability of TTE communication links in different settings, and to optimize the placement of the transmitter and receiver. 


\subsection{Introduction}

Through-the-Earth (TTE) radio has been proposed for emergency communications in a variety of subterranean or otherwise difficult to access settings, such as mines, tunnels, or multistory buildings. Frequencies between 400 and $9000 \mathrm{~Hz}$ (ULF (ultra low frequency, 300-3000 Hz) and VLF (very low frequency, 3-30 kHz) bands) are used to penetrate lossy materials such as rock and concrete over hundreds of metres. The signal has been noted to couple to elongated conductors, for example, a railroad in an underground mine, thereby increasing the lateral range. Electromagnetic interaction between elongated conductors and the ground is not by itself a novelty; it has been considered since the 1920s for communications (Carson, 1926; Stolarczyk, 1991). Nowadays, research is done for applications in MF (medium frequency, 300-3000 kHz) mine communications (Deryck, 1974; Emslie and Lagace, 1976; Wait and Hill, 1977; Stolarczyk, 1991; Li et al., 2012, 2013, 2014; Brocker et al., 2015) and geophysical detection of buried elongated conductors such as wires or pipelines (Wynn and Zonge, 1975; Wait and Umashankar, 1978; Watts, 1978; Qian and Boerner, 1995; McKenna and McKenna, 2010; McKenna et al., 2013; Naus, 2013).

The physics of coupling in the MF band have been alternatively described as a waveguide effect with the tunnel (Deryck, 1974; Stolarczyk, 1991), or as a transmission line effect in suspended wires (Li et al., 2012, 2013, 2014; Brocker et al., 2015); additionally, a full-wave analysis was also provided by Wait and Hill (1977). The conductor needs not be a metallic wire - coupling to a coal seam, which is markedly more conductive than its host rock has also been reported (Emslie and Lagace, 1976). In contrast, in the VLF band, due to the long wavelength, waveguide or transmission line approaches are eschewed in favour of treating coupling as an induction problem. 
In geophysics, elongated conductors, such as buried wires or pipelines, are considered either targets or sources of noise. Theoretical responses have been computed (e.g. Wait and Umashankar (1978); Watts (1978); Qian and Boerner (1995)), which are useful for designing systems to detect such conductors, or to attenuate noise to facilitate data interpretation (Wynn and Zonge, 1975). Geophysical instrumentation has been proposed (McKenna and McKenna, 2010; McKenna et al., 2013; Naus, 2013) for the detection of such anomalies. These systems typically use frequencies at least in the MF band to exploit the increased excitation occurring when the signal wavelength is of the same order of magnitude as the length of target.

In TTE radio applications, the coupling effect has been described by a variety of terms, including serendipitous, parasitic, or infrastructure coupling. The phenomenon is poorly understood: a method for modelling propagation has not been previously proposed, and the physics of this effect, such as how current is excited in the conductors, or what field components are present near the conductors, have not been systematically studied. In turn, the results could be used to answer several practical questions, such as the nature of signal decay along the conductor, how surrounding conductivity structures affect propagation, and how the three magnetic field components differ at a point of interest.

Our main research objective is to characterize the coupling of VLF signals to elongated conductors for application in TTE radio communications. Field experiments involving railroads and multistory buildings are performed, and a method to model the coupling is developed, and validated against the field data. 


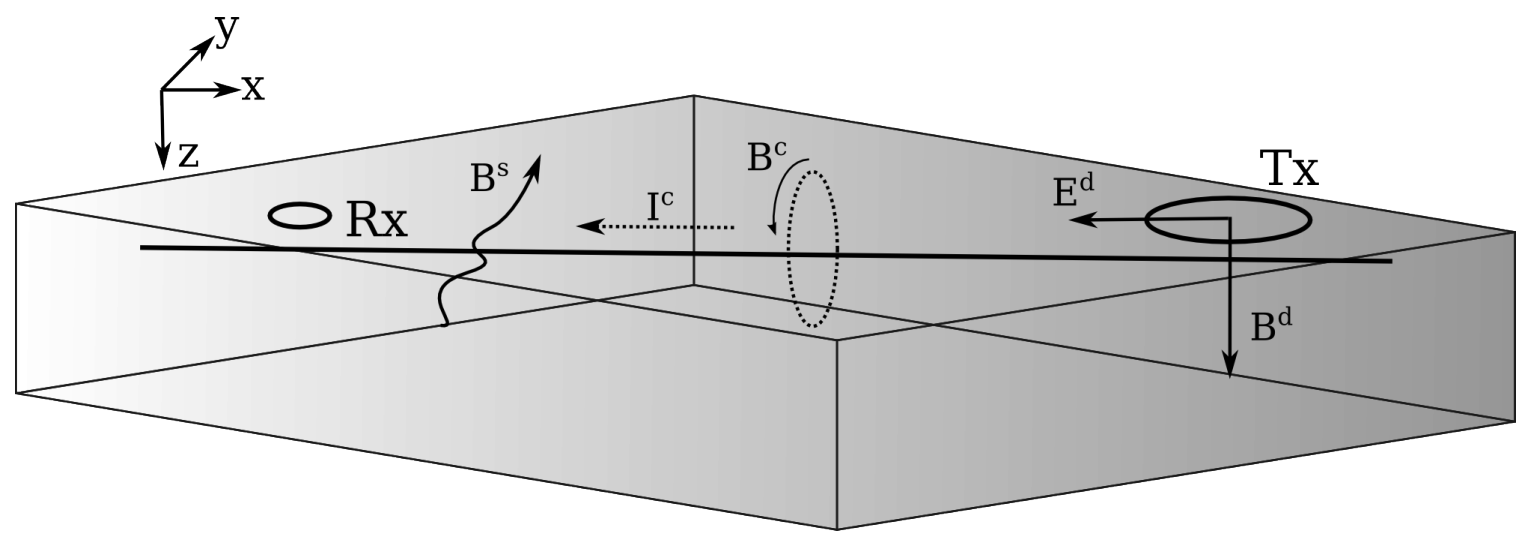

Figure 5.1: Excitation of a horizontal conductor by a vertical magnetic dipole.

\section{$5.3 \quad$ Theoretical background}

When a VLF transmitter excites a conductor whose length is much greater than its width, azimuthal and radial currents are assumed to be negligible relative to the axial current. Furthermore, the wavelength of the signal $(10-100 \mathrm{~km})$ is much greater than the length of the conductor, which makes transmission line and radiation effects negligible. As such, the problem is reduced to the induction of current along a conductor.

Consider a simplified version of a railway track: only one conductor is used (Figure 5.1). It extends underground along the x-axis, and a vertical magnetic dipole transmitter (Tx) is placed nearby. Following the work of Hill $(1988,1990)$, the conductor is excited by the primary electric field along the length of the conductor $\left(\mathbf{E}_{x}^{d}\right)$, which supports a transverse electromagnetic (TEM) mode along the conductor. Per Ampère's law, the axial current $\mathbf{I}_{x}^{c}$ generates a rotational field $\mathbf{B}^{c}$ around the conductor that decays as $1 / r$. Therefore, at the receiver $\mathrm{Rx}$, assuming $\mathbf{I}_{x}^{c}$ is sufficiently strong, two components are detected: $\mathbf{B}_{z}^{c}$ and $\mathbf{B}_{y}^{c}$. Without the conductor, $\mathbf{B}_{y}^{c}$ would not be present. Furthermore, $\mathbf{B}_{z}$ should persist more than would be expected with the typical $1 / r^{3}$ magnetic field decay in the near field. A final note: a small secondary 
magnetic field $\left(\mathbf{B}^{s}\right)$ is induced by the conductive ground.

In a single-conductor scenario, the current induced in the VLF band is minimal, because the electric field is frequency-dependent and the return path along the length of the conductor is through the ground. For two-conductor scenarios, common mode and differential mode currents are excited, which mirrors concepts of monofilar and bifilar modes of propagation suggested in the MF literature (Stolarczyk, 1991). In the monofilar case, current flows in the same direction in both conductors and returns through the ground, whereas in the bifilar case, the current flows in opposite directions in the conductors and links through the ground. A signal travels further in the bifilar case as it undergoes less ground loss. In the bifilar case, such as railroads, the opposing currents should cancel out the $B_{y}$ component between the two conductors.In the monofilar mode, which is also equivalent to the case with only one conductor, the conductivity structure of the surroundings is a control on propagation, whereas in the bifilar mode, the conductivity structure of the materials between the elongated conductors exerts a greater control. Additionally, as there are now two conductors, the fields decay away from them as $1 / r^{2}$. When a magnetic dipole is placed symmetrically about the conductors, only the bifilar mode is induced as the driving electrical field exists as loops in the plane of the transmitter. If the transmitter is offset, the currents in the two conductors will be unequal as both common and differential modes are excited.

\subsection{Methods}

\subsubsection{TTE radio system}

In this study, the CanaryTalk TTE radio system, developed by Vital Alert Communications Inc., was used. It consists of two terminals, each having a transmitter and 
Table 5.1: CanaryTalk TTE radio system specifications

\begin{tabular}{|c|c|}
\hline Transmitter dimensions & $16 \mathrm{~m}$ perimeter; maximum area $\sim 20 \mathrm{~m}^{2}$ \\
\hline Number of turns & 2 \\
\hline Current & For $100 \%$ power: $\sim 10 \mathrm{~A}$ at $4 \mathrm{kHz} ; \sim 20 \mathrm{~A}$ at $2 \mathrm{kHz}$ \\
\hline Receiver & Three-axis $(\mathrm{X}, \mathrm{Y}, \mathrm{Z})$ antenna \\
\hline Central frequency & $4 \mathrm{kHz}$ (most common); $0.4-9 \mathrm{kHz}$ (range) \\
\hline Bandwidth & $\sim 2 \mathrm{kHz}$ \\
\hline
\end{tabular}

receiver, which permits two-way communications. The specifications for this system are given in Table 5.1. Note that the transmitter current depends on the inductance of the loop; the current listed is for a minimum inductance (i.e. for a $4 \mathrm{~m} \mathrm{x} 4 \mathrm{~m}$ square loop or a circular loop with radius $\sim 2.5 \mathrm{~m}$ ).

\subsubsection{Finite-difference time-domain modelling}

A variety of analytical solutions exist to describe the interaction of a conductor with the ground, with several permutations as to whether it is bare or insulated, buried in or suspended over the ground, or even between two materials (e.g. Wait and Spies (1972); Chang and Wait (1974); Hill and Wait (1977); Wait and Hill (1977); Wait (1978); Hill (1988, 1990); Bridges (1992); Irvine (2010)). A major drawback to analytical solutions is their inability to handle arbitrary material and conductor geometry.

In our previous work (Ralchenko et al., 2015, 2016, 2017b), we developed a finitedifference time-domain (FDTD) code optimized for modelling TTE radio transmissions. In the FDTD method, complex geometries are discretized using a grid, usually rectangular, and Maxwell's equations in differential form are solved by marching forward at a given time step. For a more thorough explanation of the FDTD method, the reader is directed to, e.g. Taflove and Hagness (2005) or Elsherbeni and Demir 
(2009).

To resolve a feature in FDTD, the recommended cell size is one-tenth the wavelength of the signal in the material. Per Beggs et al. (1992), the wavelength $\lambda$ of a signal in a nonmagnetic lossy material is

$$
\lambda=\frac{\lambda_{0}}{\sqrt{\left|\frac{\sigma}{j \omega \epsilon_{0}}\right|}},
$$

where $\lambda_{0}$ is the signal wavelength in free space, $\sigma$ is the conductivity of the material, $\epsilon_{0}$ is the electric permittivity of free space, and $\omega$ is the angular frequency of the signal. Alternatively, the wavelength can be related to the skin depth $\delta$ as $\lambda=\sqrt{2} \pi \delta$, where $\delta=\sqrt{\frac{2}{\sigma \omega \mu}}$, where $\mu$ is the magnetic permeability of the material. For a ferromagnetic material where $\sigma>10^{6} \mathrm{~S} / \mathrm{m}$ and the relative permeability $\mu_{r} \approx 10^{3}$, the minimum cell size is on the order of $0.1 \mathrm{~mm}$. Such a fine grid for buildings or mines is impractical due to limitations on computer memory and execution time.

Instead of modelling the conductor as part of a material grid, a thin-wire approximation proposed by Umashankar et al. (1987); Elsherbeni and Demir (2009) can be used. This approximation places a bare, perfectly conducting thin wire along the position of the electric field $(\mathrm{E}=0)$, and updates the surrounding magnetic field based on a $1 / r$ decay away from the wire. Other approaches, namely using a material that is conductive relative to its surroundings but can still be appropriately discretized, or imposing surface impedance boundary conditions as proposed by Beggs et al. (1992) and Maloney and Smith (1992), are less efficacious. Other methods for modelling thin wires in FDTD have been proposed (e.g. Mäkinen et al. (2002); Noda and Yokoyama (2002); Baba et al. (2005)); however, the advantage of the method described in Umashankar et al. (1987); Elsherbeni and Demir (2009) for 


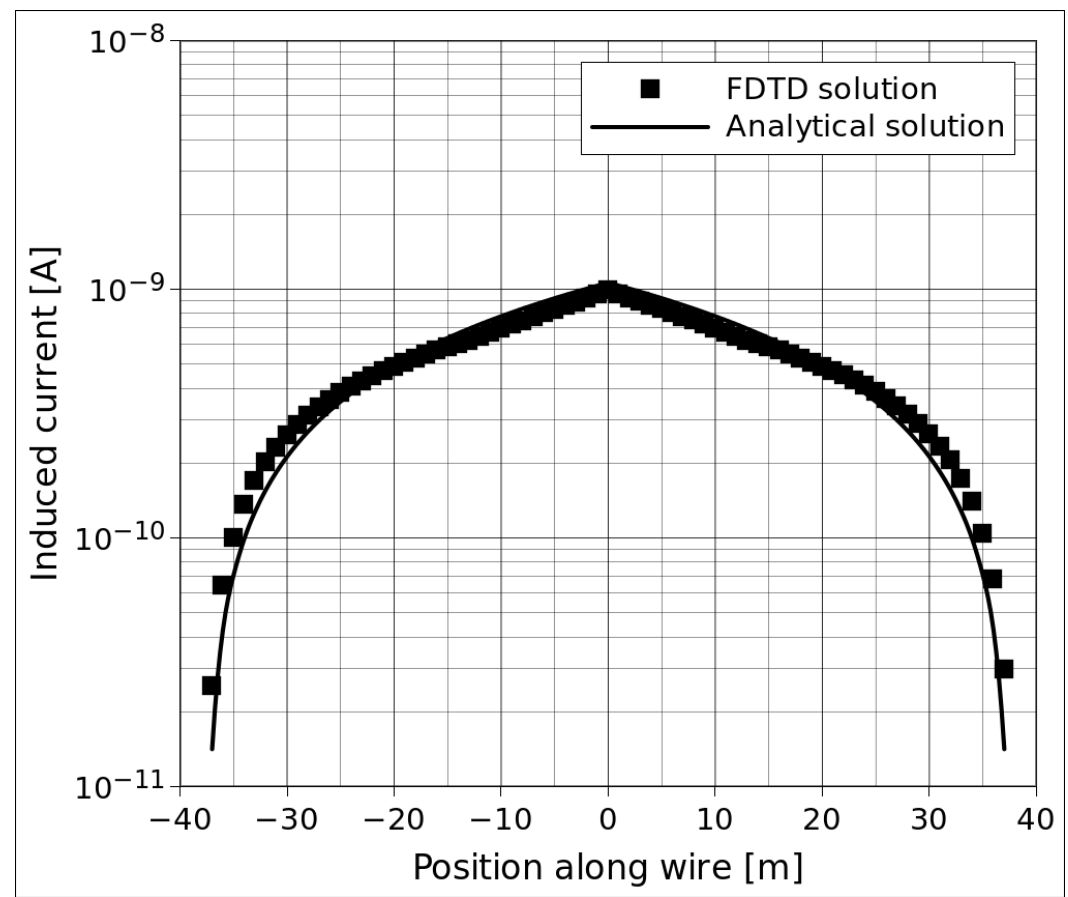

Figure 5.2: Comparison between the induced axial current at $4 \mathrm{kHz}$ in a $75 \mathrm{~m}$ long thin wire calculated analytically (after Hill (1990)) and using a thin-wire approximation for FDTD. The transmitter is placed at the origin, and centred about the wire, which extends from $-37.5 \mathrm{~m}$ to $+37.5 \mathrm{~m}$.

this application is that the conductor is not insulated and does not require considerable modifications to the FDTD algorithm. The objects of interest, such as railway tracks or elevator shafts, are grounded at regular intervals, and therefore are well suited for this approach.

The use of a thin-wire approximation for a buried conductor in FDTD is validated, following Hill (1990) and Irvine (2010). A finite 75-m long wire with radius $0.5 \mathrm{~mm}$ is placed in a medium of conductivity of $0.1 \mathrm{~S} / \mathrm{m}$; a vertical magnetic dipole of moment of $0.0029 \mathrm{Am}^{2}$, transmitting at $4 \mathrm{kHz}$ is placed $15 \mathrm{~cm}$ away. Figure 5.2 shows good agreement between the analytical and numerical results. 


\subsection{Railway tracks}

\subsubsection{Underground railway tracks}

This test was conducted in an operating underground coal mine to test the possible range of a TTE radio signal in the presence of an elongated conductor.

Located near East Lynn, West Virginia, USA, the Rockspring Development Camp Creek mine is a room-and-pillar mine approximately $100 \mathrm{~m}$ deep. The mine level and overburden are particularly resistive $(<0.001 \mathrm{~S} / \mathrm{m})$. It was dry at mine level, and in the vertical test, it was possible to receive a signal from the surface with the transmitter at a very low (5\%) power setting.

In the lateral test, the transmitter was draped over the railway tracks, and a centre frequency of $4 \mathrm{kHz}$ was used. The mine was electromagnetically noisy, in part due to a $12 \mathrm{kV}$ power cable one crosscut over. Even in these challenging conditions, it was possible to receive a signal $5.2 \mathrm{~km}$ away. Such a distance is far beyond the range of the dipole field, which decays as $1 / r^{3}$.

One explanation for this large transmission range, discussed later in subsection 5.5.3, is the low conductivity of the ground. Depending on the anchoring system used, it is possible that the railway tracks were electrically connected in some areas. As we had travelled in a cart that ran on the railway tracks, we may have created a closed loop, which would provide a longer range than an equivalent circuit that was completed by the ground.

\subsubsection{Surface railway tracks}

Further testing was done at an abandoned surface railroad at Chelsea, near Ottawa, Canada. In this test, the behavior of the three magnetic field components was investigated, as well as the propagation mode (monofilar vs. bifilar) of the current in the 
railway tracks.

The local geology consists of a resistive Precambrian crystalline basement overlain by a thin conductive soil. The railway tracks were grounded with spikes at regular intervals $(\sim 1 \mathrm{~m})$. As for the test described in subsection 5.5.1, the transmitter was draped over tracks (Figure 5.3), and a centre frequency of $4 \mathrm{kHz}$ was used.

A baseline case without the railway tracks was obtained by placing the transmitter on the ground away from the track, and measuring the signal decay by walking away orthogonally from the track (i.e. in the y-direction as per Figure 5.1). Next, the transmitter was draped over the track, and measurements were taken along the track. The comparison between the field strength for the two cases is shown in Figure 5.4. As expected, there was no difference in the $B_{x}$ component as measured along the baseline or along the railway tracks, but the $B_{y}$ and $B_{z}$ components along the track persisted for longer, and decayed at a rate of $11 \mathrm{~dB} / 100 \mathrm{~m}$ compared to the baseline decay rate of $47 \mathrm{~dB} / 100 \mathrm{~m}$.

In a bifilar propagation mode, the $B_{y}$ component is expected to be null between the tracks. In the field, the $B_{y}$ got markedly weaker but not entirely null. To explain this result, the signal decay going away from each track was measured separately. The signal attenuated faster going away from one track compared to the other; therefore, the induced currents must also be unequal, perhaps due to difference in grounding, and would not entirely suppress $B_{y}$ between the railway tracks.

A second experiment was performed on the surface railroad to investigate the propagation mode. A copper wire was electrically connected to both railway tracks, and measurements were taken before and after the connection was established. There was no difference in signal strength before the connection, whereas after, there was a sharp loss $1 \mathrm{~m}$ away with complete loss of reception $10 \mathrm{~m}$ away. This test confirms the presence of a bifilar mode as there was no change in signal strength before the 


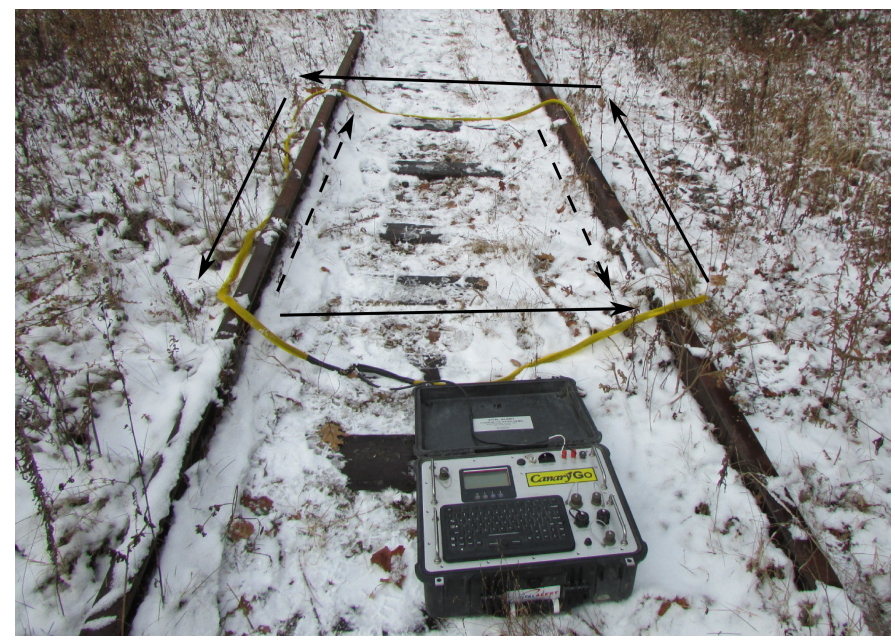

Figure 5.3: Equipment setup along surface railway tracks at Chelsea, Canada. The transmitter is draped over the tracks which induces current along the tracks (dashed arrows) in the direction opposite to those in the loop (solid arrow) as per Lenz's law.

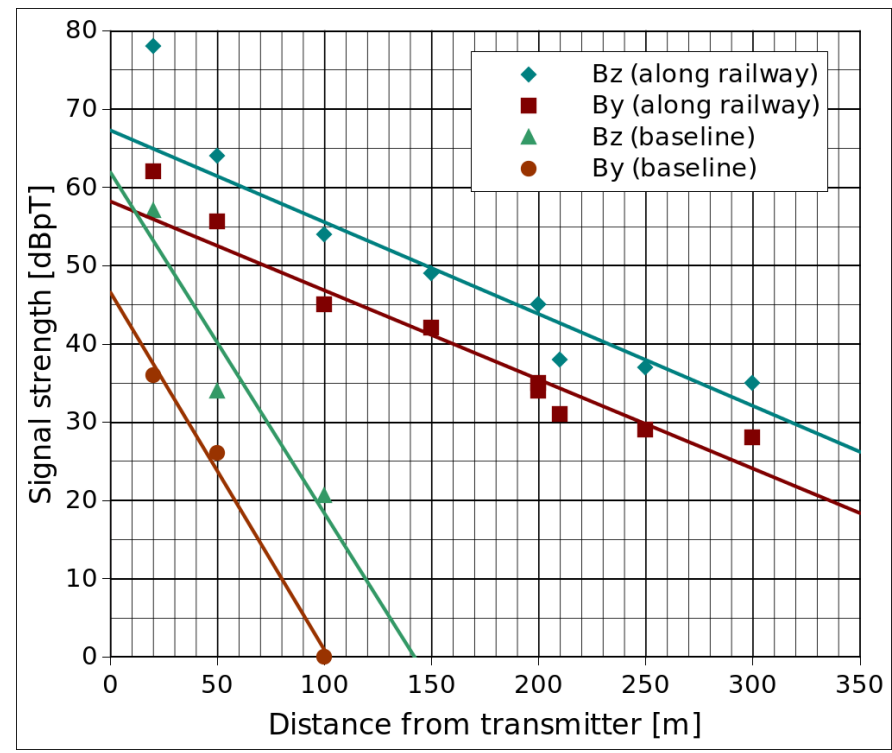

Figure 5.4: Signal decay along surface railway tracks at Chelsea, Canada. Data was gathered next to one of the tracks. Signal strength is presented in dB relative to $1 \mathrm{pT}$.

connection. The loss of signal after the connection indicates a considerable induced axial current in the railway track, which was also suggested by the presence of the $B_{y}$ and $B_{z}$ components combined with the absence of the $B_{x}$ component along the railway 
tracks. Finally, the electrical connection would not have affected signal strength had a monofilar mode been excited, as there would exist no potential difference between the two tracks, unlike the case for the bifilar mode.

\subsubsection{Numerical modelling}

Modelling the coupling of signal to railway tracks is simpler than for other metallic structures: it is generally known where the conductors are located. The purpose of the modelling is two-fold: first, to reconcile the field data to a model, and second, to make predictions for different situations.

Relative to the transmitter, the railway tracks can be considered infinitely long. To terminate the FDTD grid, the convolutional perfectly matched layer (CPML) absorbing boundary condition $(\mathrm{ABC})$ is used, and the thin wires that represent the tracks are extended into the CPML. The mechanical analogy of an ABC is an anechoic chamber. The ground is represented by a homogeneous halfspace.

To model coupling to railway tracks, a current loop is placed over the thin wires, and is excited at $4 \mathrm{kHz}$. Results for transmitter parameters used in Chelsea, Canada (subsection 5.5.2) are presented in Figure 5.5 as graphs of signal strength and decay with respect to the vertical magnetic field $\left(B_{z}\right)$ observed $100 \mathrm{~m}$ away from the transmitter for ground conductivities ranging from $0.001 \mathrm{~S} / \mathrm{m}$ to $1 \mathrm{~S} / \mathrm{m}$. For a ground conductivity of $0.01 \mathrm{~S} / \mathrm{m}$, there is agreement between the observed and modelled signal strengths and decay rates.

In the model, there is no contribution to the $B_{y}$ component from the coupling effect directly between the tracks; instead, a very small secondary field induced by the ground (i.e. $B^{s}$ in Figure 5.1) is observed. Removing one of the tracks from the model resulted in a marked increase in $B_{y}$, which further confirms that unequal currents were induced in the railway tracks at Chelsea. 


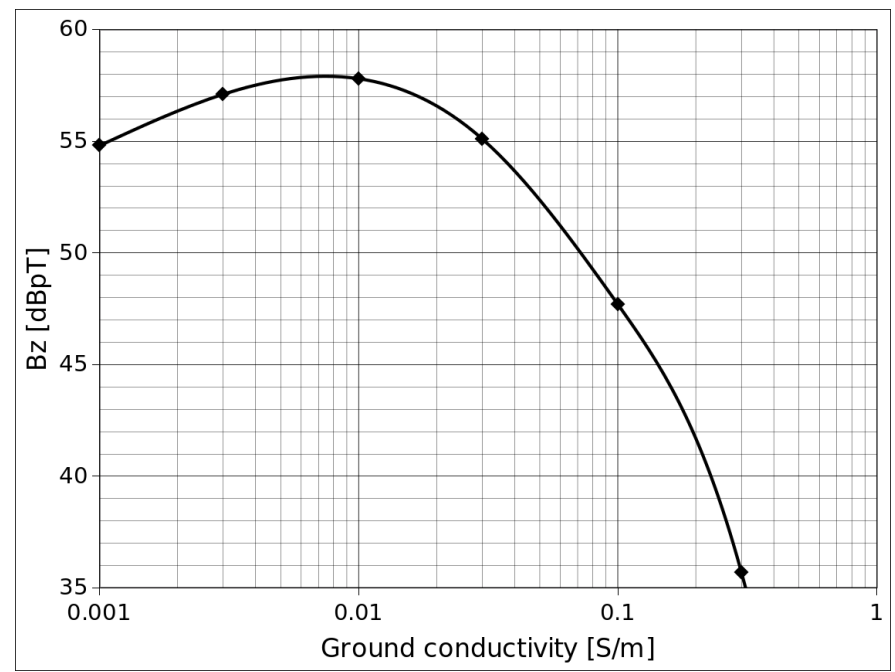

(a)

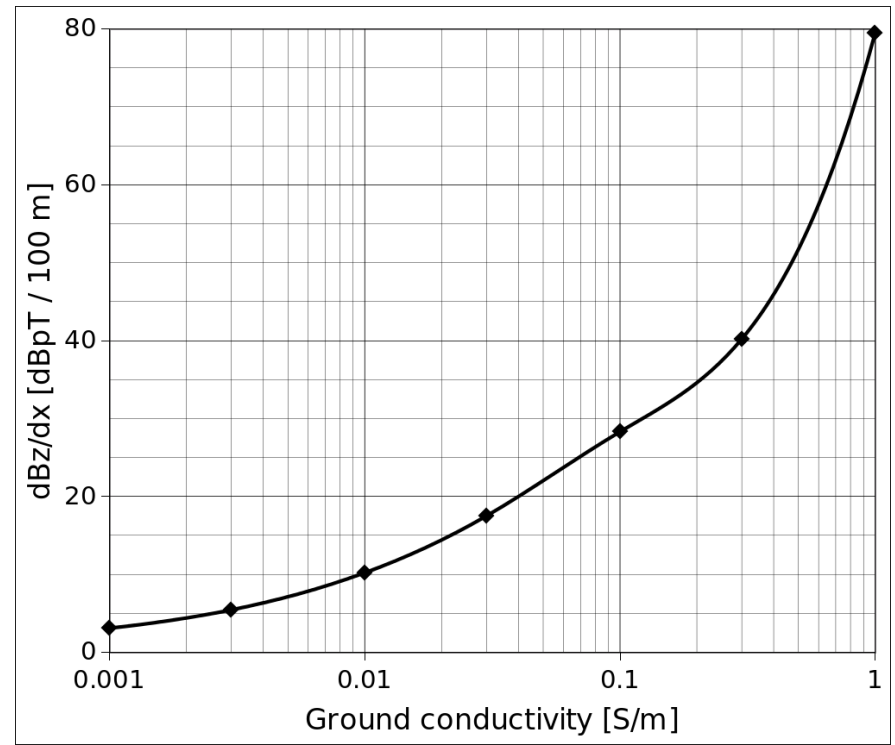

(b)

Figure 5.5: Signal strength (a) and rate of change with distance along the railway tracks (b) for the vertical magnetic field $\left(B_{z}\right) 100 \mathrm{~m}$ away from the transmitter for various ground conductivities.

Coinciding with the apex of the curve shown in Figure 5.5a, a ground conductivity of approximately $0.01 \mathrm{~S} / \mathrm{m}$ is optimal for achieving a communication link by coupling electromagnetic signal to a railway track $100 \mathrm{~m}$ away from the transmitter. When the track is in a highly resistive medium, the ground link cannot support the excitation of 
a bifilar mode of propagation. However, as the conductivity of the medium increases, more current leaks into the ground until this loss negates the additional signal gained by having a current excited along the railway tracks.

\subsection{Elevator shaft}

During tests of TTE radios in multistory buildings, it was observed that the signal couples to conductors in elevator shafts. These include guide rails or other vertical components going down the shaft, horizontal structures such as guide rail reinforcements, or even the elevator car (Strakosch, 1998; Janovský, 1987).

Field work was performed in the 22-story Dunton Tower on the campus of Carleton University, Ottawa, Canada. The building has four elevators that are grouped in pairs on either side of a central waiting area (Figure 5.6a). In the FDTD model, the floors and walls are represented as sheets of a given conductance (product of conductivity and thickness). The conductance of these structures depends on the rebar in the concrete, both in terms of quantity and geometrical arrangement. The elevators are represented by a thin wire running along the height of the building. A horizontal wire connects neighbouring elevators at the 21st level, which is where the transmitter was placed (Figure 5.6b).

Data acquisition was performed with a transmitter $(6 \mathrm{~m} \mathrm{x} 2 \mathrm{~m}$, total moment $\sim$ $250 \mathrm{Am}^{2}$ ) placed in the waiting area (Figure 5.6a). The signal strength was measured in one-metre increments one metre away from the elevator as shown in Figure 5.6a.

A conductance model of $10 \mathrm{~S}$ floors and $1 \mathrm{~S}$ walls was fitted to the field data. The comparisons of observed and computed field strengths for the lateral $\left(B_{x}\right.$ and $\left.B_{y}\right)$ components is given in Figure 5.7. The vertical field $\left(B_{z}\right)$ is not shown as it could not be resolved in the noise environment of no less than $30 \mathrm{dBpT}$. The uncertainty 


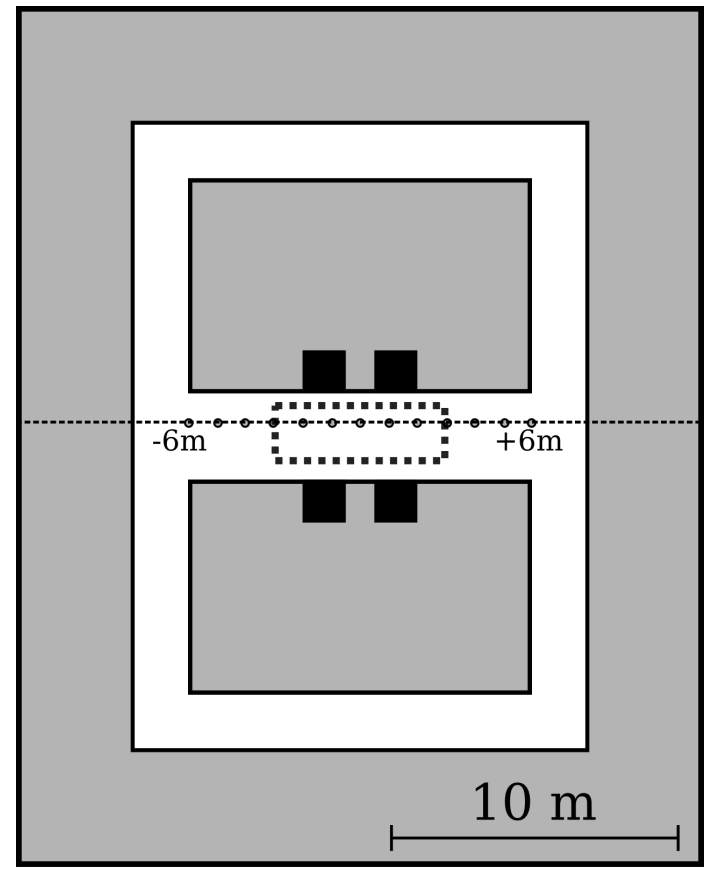

(a) Floor plan

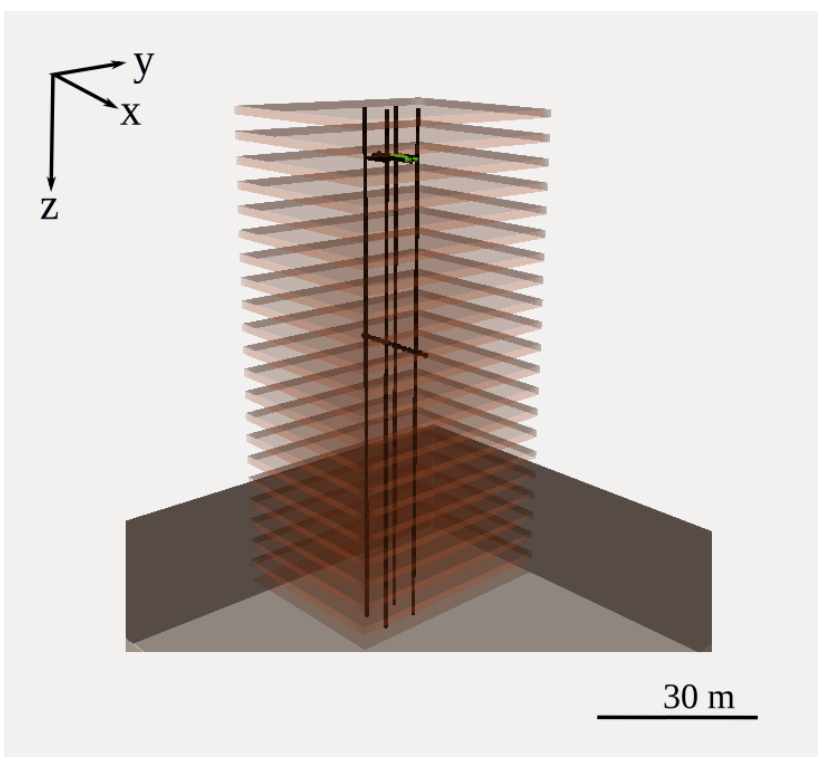

(b) 3-D model with walls removed

Figure 5.6: Architectural layout of Dunton Tower. Office space is shaded, corridors are white, and elevator shafts are black. The transmitter and receiver locations are indicated by the coarse dashed line and small circles, respectively. The position of the plane sampled in Figures 5.8 and 5.9 is indicated by a fine dashed line in (a). 
in the arrangement of conductors in the elevator shaft makes modelling more difficult compared to railway tracks. Furthermore, there exist many areas in such a building where electrical connections can be made with the elongated conductors in the elevator shaft, which further contributes to the uncertainty. For example, the $B_{y}$ profile for level 12 is somewhat asymmetric, which can likely be explained by variations in the geometry of the conductors within the elevator shaft. In the $B_{x}$ component, a null is predicted between the elevators, and a strong decrease in signal is observed at the midpoint. The small field at the midpoint arises from uncertainty in position, transmitter layout, noise, and contributions of other conductivity structures.

There are several marked similarities between the observed and computed data. Between two elevators, there is a dip in $B_{x}$ but a peak in $B_{y}$, and a peak in $B_{x}$ exists instead near the individual elevators. The increased signal is observed only near the elevators, and it quickly decays to the baseline when going away. In Figures 5.8 and 5.9, the three magnetic field components are shown as a slice in the $x z$ plane (cf. Figure 5.6a), with and without the elevators, respectively. As seen in the field, the $B_{x}$ and $B_{y}$ components persist much further than the $B_{z}$ component, in the presence of the elevators. The $B_{x}$ component is strongest near each elevator and persists further down the building when the elevators are taken into account. The $B_{y}$ component is particularly strong between the two elevators, and is not present in that particular plane when the elevators are not modelled. Finally, as expected from the alignment of the transmitter relative to conductors, the $B_{z}$ does not change from the baseline, and it is for this reason that the lateral components persisted further than the vertical one. This analysis shows that for a communication link in a multistory building, placing the transmitter near an elevator increases vertical range, provided the receiver is placed near the same elevator. 


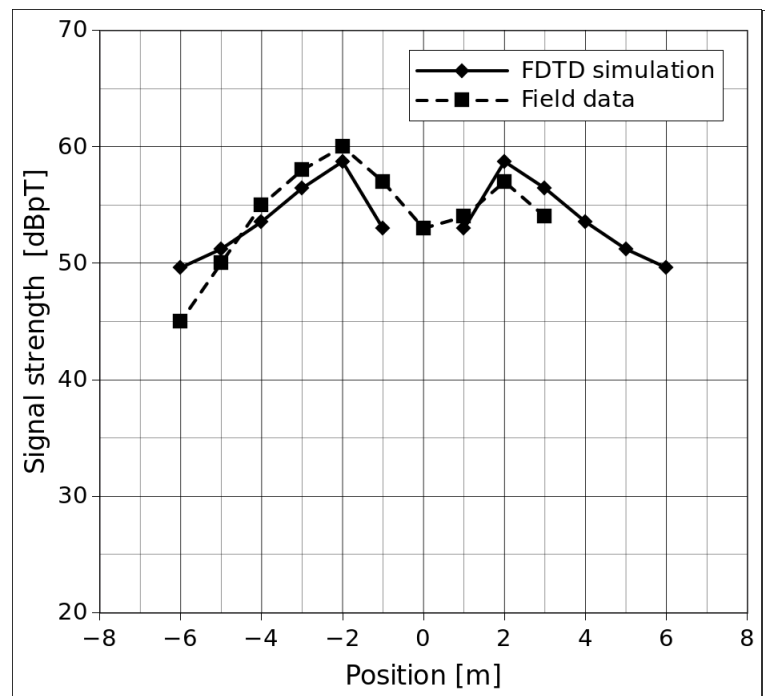

(a) $B_{x}$, level 13

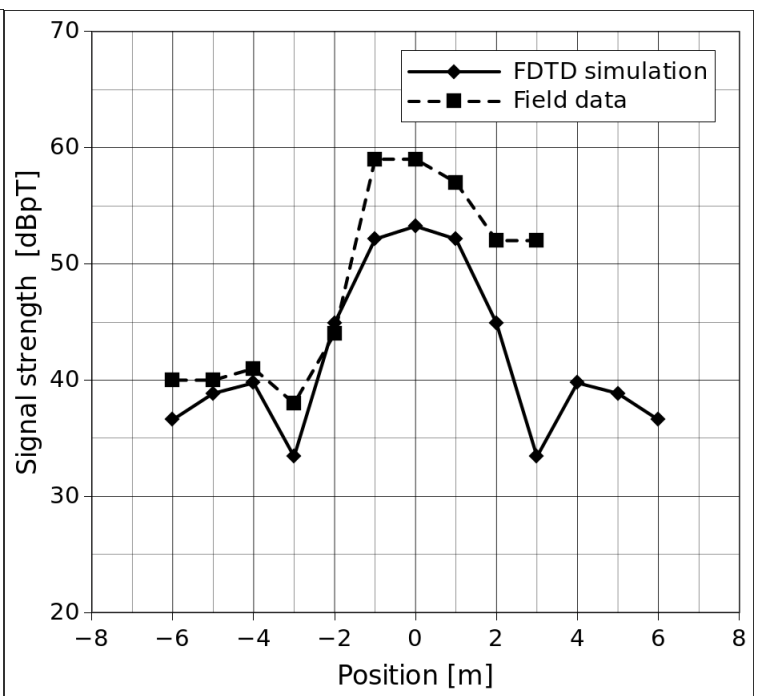

(b) $B_{y}$, level 13

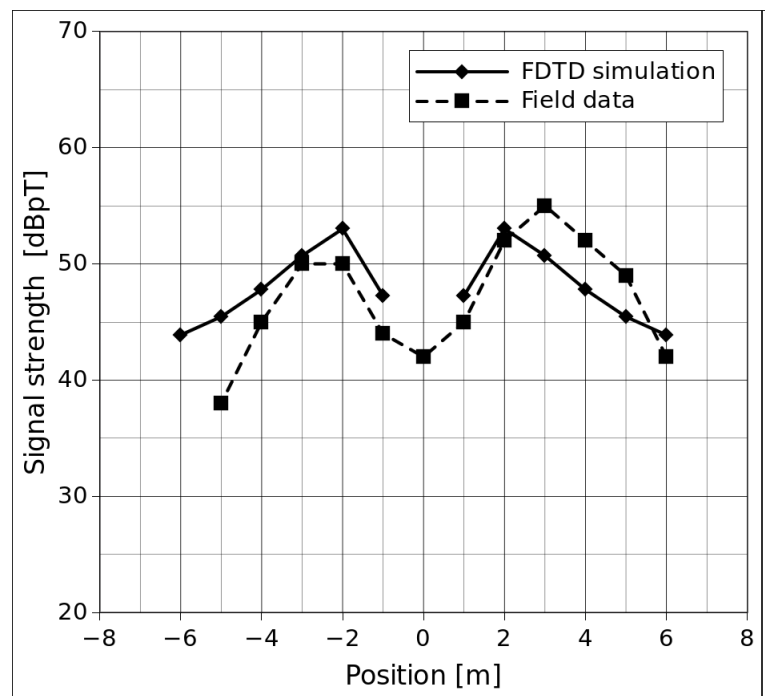

(c) $B_{x}$, level 12

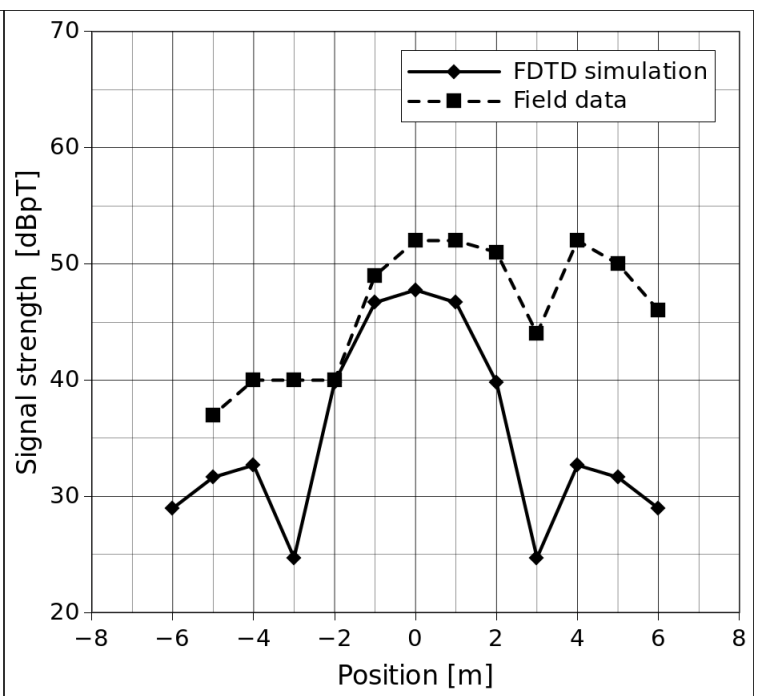

(d) $B_{y}$, level 12

Figure 5.7: Comparison of signal strength in Dunton Tower measured in the field and computed using FDTD. Two levels are shown to demonstrate consistency in the results.

\subsection{Conclusion}

In this paper, experimental and numerical results for the coupling of a TTE radio signal to elongated conductors have been presented. Both data sets suggest that a communication link can be successfully established by exploiting the presence of 


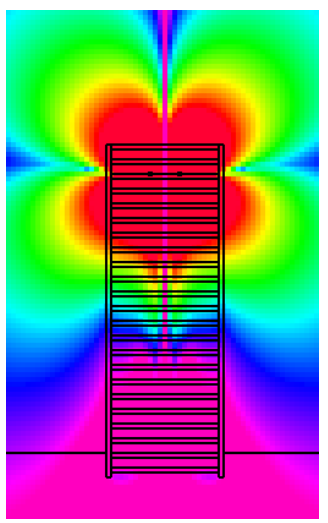

(a) $B_{x}$

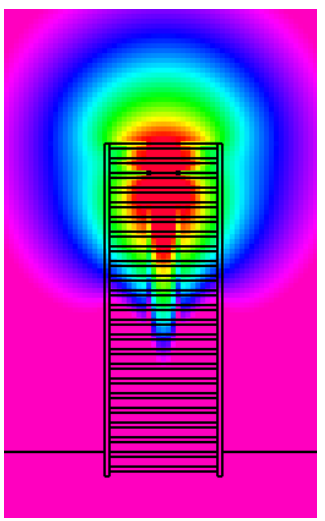

(b) $B_{y}$

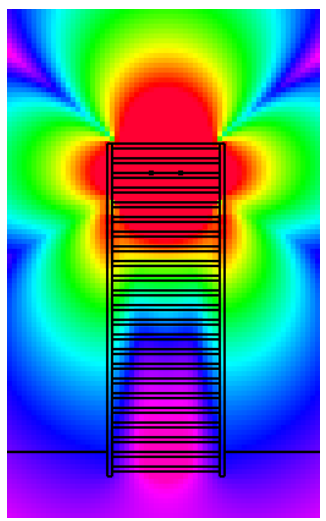

(c) $B_{z}$

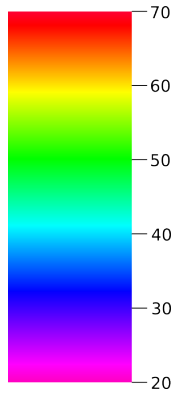

Figure 5.8: Signal strength $(\mathrm{dBpT})$ in Dunton Tower matched to field data for small loop.

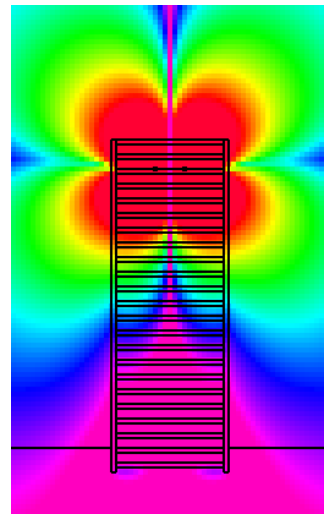

(a) $B_{x}$

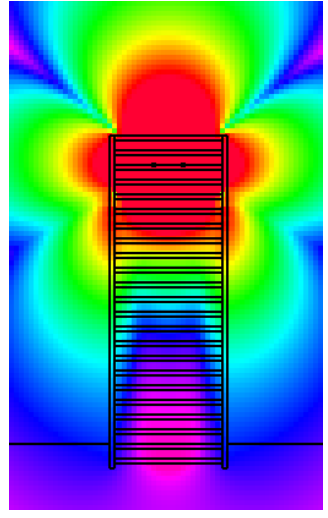

(b) $B_{z}$

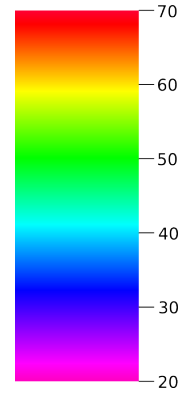

20

Figure 5.9: Signal strength (dBpT), baseline with no elevators, in Dunton Tower using small loop. The field is sampled such that the $B_{y}$ component is null.

elongated conductors. It was shown that currents were induced in the conductors, which yielded a characteristic magnetic field structure where components orthogonal to the conductor persisted much farther than for a baseline case. For railway tracks, it was shown that a bifilar mode was excited. Finally, a multistory building with elevators was successfully modelled. This experiment showed that the vertical range of TTE radios in such buildings can be greatly extended by placing the transmitter near the elevator while the receiver is next to the same elevator. 
The FDTD models are a powerful tool not only for reconciling theoretical and observed data, but to make predictions for the viability of a link when certain system parameters are changed (e.g. transmitter orientation) or for an entirely new setting. The capability of the FDTD method to model an arbitrary material geometry allows for simulation to be made for very disparate environments. The settings described here are but examples where such coupling is possible, and further research could be done to test the proposed methodology in locations with different conductivity structures, such as underground mine shafts.

With some knowledge of the nearby conductivity structures, the deployment of TTE communications systems can be readily optimized, based on theoretical considerations and numerical experiments, in particular with regards to the placement of the transmitter. For example, the magnetic field from a monofilar excitation will persist farther from the conductors, but with more of a return path through the ground, the signal range along the conductors would suffer. The transmitter could be placed to exploit variations in the conductivity near the elongated conductors, observable as changes in material, as the bifilar mode is more sensitive to structures in the volume between the elongated conductors, whereas the monofilar mode is sensitive to structures around the conductors. In cases with several conductors, such as in elevator shafts, FDTD is particularly powerful as the position of the transmitter can be chosen prior to deployment to target a specific area for improved signal strength.

\subsection{Acknowledgments}

The authors wish to thank Alpha Natural Resources for granting access to the Rockspring Development Camp Creek mine, and Vladmir Puzakov, Michael Homer, and John Steckley for their assistance during field work at the mine site. Brian Du and 
Peter Kwasniok are thanked for their assistance and input during preliminary testing. We thank Peter Oliver and Andrew Hay for their help during field work at Chelsea. Carling Walsh is thanked for her assistance with the field work in Dunton Tower. Comments from two anonymous reviewers significantly improved this article. This research project is funded by the Natural Sciences and Engineering Research Council of Canada (NSERC) and Vital Alert Communication, Inc., through an Industrial Postgraduate Scholarship to M. Ralchenko. 


\section{Chapter 6}

\section{Near-field VLF electromagnetic signal propagation in multistory buildings}

\subsection{Abstract}

In this paper, we present modelling of VLF (very low frequency; 3-30 kHz) throughthe-Earth (TTE) radio transmissions in four multistory buildings using the finitedifference time-domain (FDTD) method. These structures are generally made of reinforced concrete, whose steel content is the most important control on bulk conductivity. The floors and walls are represented as sheets of a given conductance, based on the fact that electromagnetic geophysical methods will only resolve conductive sheets in terms of their conductance as opposed to both conductivity and thickness. We validate this approach first using synthetic data, and second, by comparison with field data. Conductance models were derived from observed data for a variety of propagation environments, including reinforced concrete, steel floors, and basements, and for transmission into, out of, or within a building. The models were able to account for non-intuitive observations, such as increases in signal strength 
near the edges of buildings and in localized areas in between buildings. Conductances obtained for the floors and walls could be used as representative values in future forward modelling to assess the viability of, or optimize a TTE radio link in a multistory building.

\subsection{Introduction}

Through-the-Earth (TTE) radio has been suggested for emergency communications in a variety of settings such as multistory buildings, underground mines, or tunnels. It uses frequencies between 400 and $9000 \mathrm{~Hz}$, which correspond to the ULF (ultra low frequency; 300-3000 Hz) and VLF (very low frequency; 3-30 kHz) bands, to reduce attenuation in lossy materials. There are several benefits of using TTE radio in multistory buildings. Some locations are difficult to reach by conventional wireless means, such as basements. In emergencies, wired or wireless communications systems could become disabled; furthermore, mobile telephone networks could be overloaded by the high volume of calls. In situations where the existing wireless infrastructure remains operational, it can be integrated with a TTE radio system to access difficultto-reach locations.

Preliminary signal propagation tests in multistory buildings show strong attenuation after as little as 3-4 levels. ${ }^{1}$ The multistory buildings of interest in this study are constructed out of concrete reinforced with steel bars, termed rebar, arranged in a gridded structure. By itself, the conductivity of concrete is similar to that of earth materials, and depends on age and saturation, but does not typically exceed $0.1 \mathrm{~S} / \mathrm{m}$ (Karhunen, 2013). This value is not representative of the whole floor, as its bulk conductivity depends on the presence of rebar. Signal propagation is dominated largely

\footnotetext{
${ }^{1}$ In this paper, a "level" spans an interval in a building, whereas a "floor" is a physical structure.
} 
by two effects. First, geometric spreading attenuates the signal as $1 / r^{3}$. Second, eddy currents are induced in the conductive floors which further attenuates the magnetic fields. The position of the transmitter relative to the building is an additional factor of interest; if the transmitter is outside, magnetic field lines not penetrating the building will not be attenuated by the conductive floors or walls. To reliably account for these effects, a numerical model is needed, as has previously been done for analyzing TTE radio transmissions (Ralchenko et al., 2015, 2016, 2017b,a).

Near-field VLF transmissions in multistory buildings have not been considered at any length in the literature. Smith (1978) considered signal propagation between $20 \mathrm{kHz}$ and $500 \mathrm{MHz}$ into a variety of buildings. The major distinction between those experiments and those reported in this paper is that Smith (1978) used a farfield VLF source $\left(1 / r\right.$ geometric decay) whereas we use near-field signals $\left(1 / r^{3}\right.$ decay). In contrast, the measurement of RF (radio frequency) propagation into, out of, or within buildings is not a particularly novel problem - the literature is replete with examples such as early attempts at mobile telephony at $35 \mathrm{MHz}$ and $150 \mathrm{MHz}$ Rice (1959), VHF (very high frequency; 30-300 MHz) and UHF (ultra high frequency; 300-3000 MHz) propagation tests in urban areas (Blaunstein and Levin, 1996), or the design of a system for personnel location using a wideband signal spanning from 550-700 MHz (Cyganski et al., 2003).

The objectives of this study are: (1) to develop a method to predict signal strength from near-field VLF transmissions in multistory buildings; (2) to experimentally validate the modelling approach; and (3) to investigate differences in signal propagation for different conductivity distributions. The body of this paper is divided into five sections: the methodology is described first, and is followed by case studies for four different buildings. Each of the case studies demonstrates successful modelling of a given propagation environment, which includes reinforced concrete floors, steel floors, 
basements, and transmissions into, out of, and within a building.

\subsection{Methodology}

\subsubsection{Through-the-Earth radio system}

In this study, we used the CanaryTalk TTE radio system, developed by Vital Alert Communication Inc., to acquire field data. Each terminal has a transmitter and receiver, which supports a time-division duplex (TDD) link with another terminal. The transmitter has a $16 \mathrm{~m}$ perimeter (maximum area $\sim 20 \mathrm{~m}^{2}$ ) with two turns of wire. At $4 \mathrm{kHz}$, the most common central frequency used, for a minimum inductance (i.e. $4 \mathrm{~m} \mathrm{x} 4 \mathrm{~m}$ square loop or circular loop with radius $\sim 2.5 \mathrm{~m}$ ), a 10 A current is supported. The receiver is a three-axis $(\mathrm{X}, \mathrm{Y}, \mathrm{Z})$ antenna. To improve portability, the transmitter loop is designed to be wrapped around the three-axis antenna when not in use.

\subsubsection{Finite-difference time-domain modelling}

In our previous work (Ralchenko et al., 2015, 2016, 2017a,b), we developed a finitedifference time-domain (FDTD) code optimized for modelling TTE radio transmissions. In the FDTD method, complex geometries are discretized using a grid, usually rectangular, and Maxwell's equations in differential form are solved by marching forward at a given time step. A more thorough explanation of the FDTD method can be found in Taflove and Hagness (2005) or Elsherbeni and Demir (2009). In Ralchenko et al. (2017a), we used FDTD to model the coupling of the TTE radio signal to elongated conductors; one application studied in particular was coupling to conductors in elevator shafts in multistory buildings. FDTD is a powerful method to simulate 
TTE radio transmissions in multistory buildings as it can efficiently model arbitrary geometries. In the literature, signal propagation in multistory buildings has been modelled using FDTD, but at much higher frequencies (Dalke et al., 2000; Austin et al., 2011; Austin, 2016). Dalke et al. (2000) calculated reflection and transmission coefficients for scattering from a reinforced concrete wall at 100-6000 MHz. Austin et al. (2011) modelled propagation at $1.0 \mathrm{GHz}$ while incorporating objects in an office setting, such as furniture, and later improved their code to include fire as a material (Austin, 2016).

\subsubsection{Thin sheets}

It is impractical to discretize a rebar mesh within a geometrical domain on the scale of a multistory building. Smaller FDTD cells necessitate a smaller time step to ensure numerical stability, which increases memory use. Hence, such an approach will have an exorbitant execution time. Instead, the floor is modelled as a thin conductive sheet. In inductive electromagnetic geophysical surveys, which are highly analogous to TTE radio experiments given the transmitter design and frequency range used (Ralchenko et al., 2016), sheet-like targets, which have a minimal thickness relative to their lateral extent, are resolved in terms of their conductance $\mathcal{G}$ (Keller, 1988), which is the product of conductivity $\sigma$ and thickness $d$ :

$$
\mathcal{G}=\sigma d
$$

This approach has secondary benefits. A modelled floor can be slightly thickened provided that its conductivity is adjusted to yield the appropriate conductance. In addition, small conductive objects, which are difficult to resolve individually with a TTE radio system, can be treated as part of the conductive floors or walls. A final 
consideration is that the cell size should be a fraction of the skin depth $\delta$ of the material. For most cases with a VLF signal, this quantity is a function of the angular frequency $w$, magnetic permeability $\mu$ and conductivity $\sigma$ :

$$
\delta=\sqrt{\frac{2}{\omega \mu \sigma}}
$$

If the skin depth is smaller than the cell thickness, the material might not be properly resolved, and an erroneously strong signal would be predicted.

\subsubsection{Synthetic data}

To validate that the conductance of a floor is the predominant control on the attenuation of a signal in a building, as opposed to purely floor thickness or conductivity, several simulations were run over with floors of constant conductance, but their thickness or conductivity varied. As an example, results are shown for a six-story building with a footprint of $50 \mathrm{~m} \times 50 \mathrm{~m}$ and levels separated by $4 \mathrm{~m}$, with a floor conductance of $10 \mathrm{~S}$. The transmitter was placed in the centre of the building on level 6 ; this location minimized edge effects. For each simulation, the conductivity and thickness were varied to test whether signal propagation is dependent on conductance. Additionally, different vertical cell sizes (i.e. $d z$ ) were tried to observe whether using more cells to represent the floor made a difference. The horizontal cell sizes (i.e. $d x$ and $d y$ ) were kept constant at $100 \mathrm{~cm}$. The results and parameters used for each of the 11 simulations, labelled A to J, are given in Table 6.1.

Results for simulations A-E show that the number of cells used to represent the floor has little effect on the computed signal strength. The consistency of the results of simulations $\mathrm{F}-\mathrm{J}$ show that, as expected, the floor conductance is the primary control on signal strength. Note that simulation $\mathrm{F}$ is not a realistic model of a multistory 
Table 6.1: Conductance model specifications and comparison of signal strength $\left(B_{z}\right.$ $[\mathrm{dBpT}])$. The transmitter is located on L6.

\begin{tabular}{ccccccccccc} 
Simulation & $\sigma[\mathrm{S} / \mathrm{m}]$ & $d[\mathrm{~cm}]$ & $d z[\mathrm{~cm}]$ & Cells per floor thickness & L6 & L5 & L4 & L3 & L2 & L1 \\
\hline A & 10 & 100 & 100 & 1 & 140 & 119 & 101 & 87 & 76 & 67 \\
B & 10 & 100 & 50 & 2 & 140 & 119 & 101 & 87 & 76 & 66 \\
C & 10 & 100 & 25 & 4 & 140 & 119 & 101 & 87 & 76 & 65 \\
D & 10 & 100 & 20 & 5 & 140 & 119 & 101 & 87 & 76 & 64 \\
E & 10 & 100 & 10 & 10 & 140 & 119 & 101 & 87 & 76 & 64 \\
F & 2.5 & 400 & 100 & 4 & 140 & 119 & 101 & 87 & 76 & 64 \\
G & 20 & 50 & 25 & 2 & 140 & 119 & 101 & 87 & 76 & 64 \\
H & 25 & 40 & 20 & 2 & 140 & 119 & 100 & 87 & 76 & 65 \\
I & 40 & 25 & 25 & 2 & 140 & 119 & 100 & 87 & 76 & 64 \\
J & 100 & 10 & 10 & 1 & 140 & 119 & 100 & 87 & 76 & 65
\end{tabular}

building, as it does not include free space between the floors. The set of numerical simulations in Table 6.1 confirms that representing a multistory building in terms of thin sheets of given conductance is a viable approach for modelling TTE radio propagation in such structures.

\subsubsection{Conductance model}

A conductance model for each of the four buildings studied was fitted based on the field data. Obtaining representative conductances for the floors and walls is of particular interest as they can be used for future forward modelling of signal strength when setting up an operational TTE radio link. To represent a building, we assume that it consists of sheets that represent both floors and walls, albeit of different conductances. Depending on the building, the ground conductivity (e.g. above ground to below ground link) or additional structures (e.g. elevator shafts) are also accounted for. A model is obtained by trial-and-error, where several FDTD simulations with 
varying conductances are run to minimize the deviation between observed and modelled signal strengths. Visual comparisons of the data, which are plotted as observed or modelled signal strength against distance from the transmitter, are also done. In Ralchenko et al. (2017b), a similar approach was used to fit a conductivity model for a mine overburden based on TTE radio signal strengths. Typically, conductance is estimated to one significant digit, as there are several sources of uncertainty in the assumed model, which include geometry, the presence of conductive elements in the building that are not readily apparent, and even signal coupling to elongated conductors (Ralchenko et al., 2016).

\subsection{Case studies}

Results for four different buildings are presented. These examples were chosen based on differences in construction and possible locations of the transmitter and receiver. In the parking garage, located on the campus of Carleton University, Ottawa, Canada, the TTE radio signal was transmitted within the building, similar to the setup of the synthetic experiment. In the hotel, located at a military base, the signal was transmitted into the building. The embassy, also at the military base, featured a similar transmitter-receiver setup to that of the hotel, except that this building had metallic floors. Finally, the signal was transmitted out of the Loeb Building (Carleton University); a further point of interest was that, in this case, the transmitter was located two levels underground.

\subsubsection{Parking garage}

A concrete multi-level parking garage is located on the campus of Carleton University, Ottawa, Canada (Figure 6.1). It is composed of two halves, each with surface 


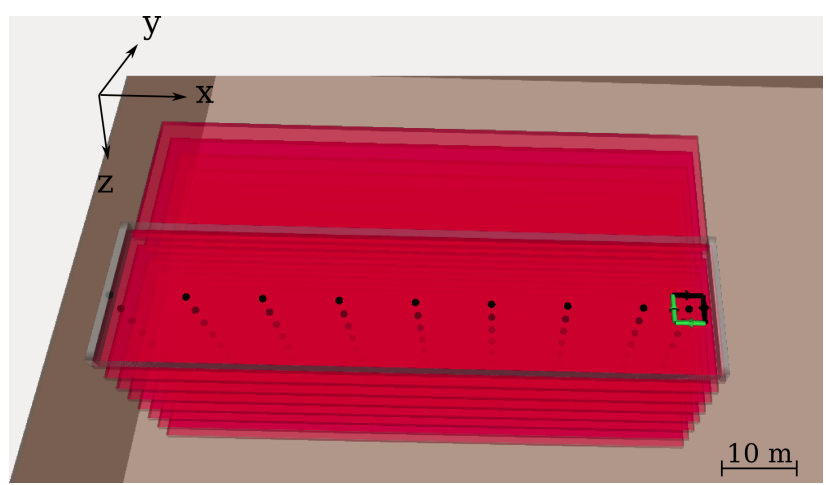

(a) Top view

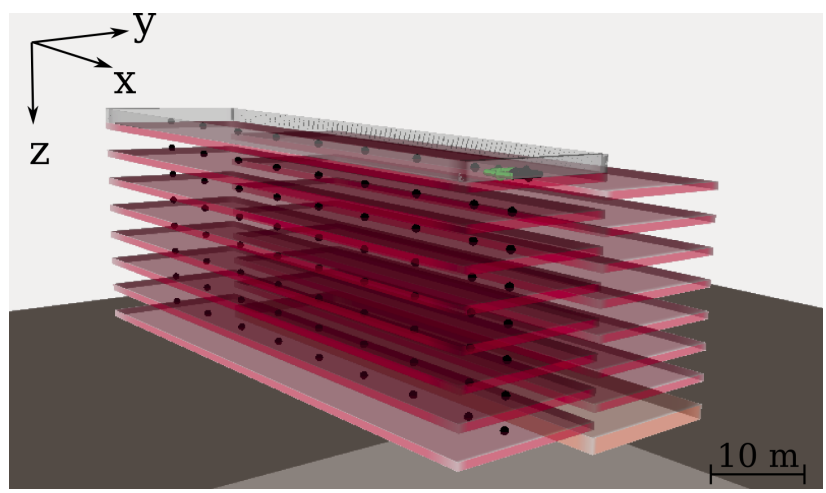

(b) Side view

Figure 6.1: Model of parking garage; the building footprint is $80 \mathrm{~m} \mathrm{x} 40 \mathrm{~m}$. Black dots represent receiver locations; the black and green loop represents the transmitter.

dimensions of $80 \mathrm{~m} \times 20 \mathrm{~m}$, and whose floors are offset by $2 \mathrm{~m}$. The walls on levels below the roof are generally thin and short, whereas the wall on the roof is thicker and taller. Data were collected from one half of the structure. A transmitter was placed on the roof, $2 \mathrm{~m}$ from the edge of the garage and centred about the y-axis, and measurements were taken along the $\mathrm{x}$-axis.

Figure 6.2 compares observed and modelled signal strength profiles one level below the roof. A conductance of $30 \mathrm{~S}$ was used for the floor. Ambient noise levels were 20-25 dBpT, and the receiver saturation threshold is $76 \mathrm{dBpT}$. For the $B_{x}$ and $B_{z}$ components of the magnetic field, receiver saturation causes differences between the observed and modelled signal strengths at distances $\mathrm{x}<15 \mathrm{~m}$. The $B_{y}$ component 


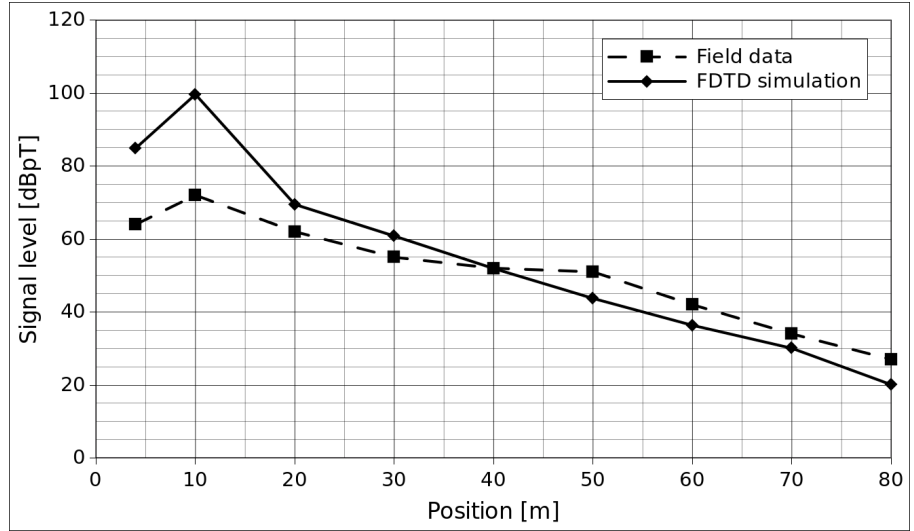

(a) $B_{x}$

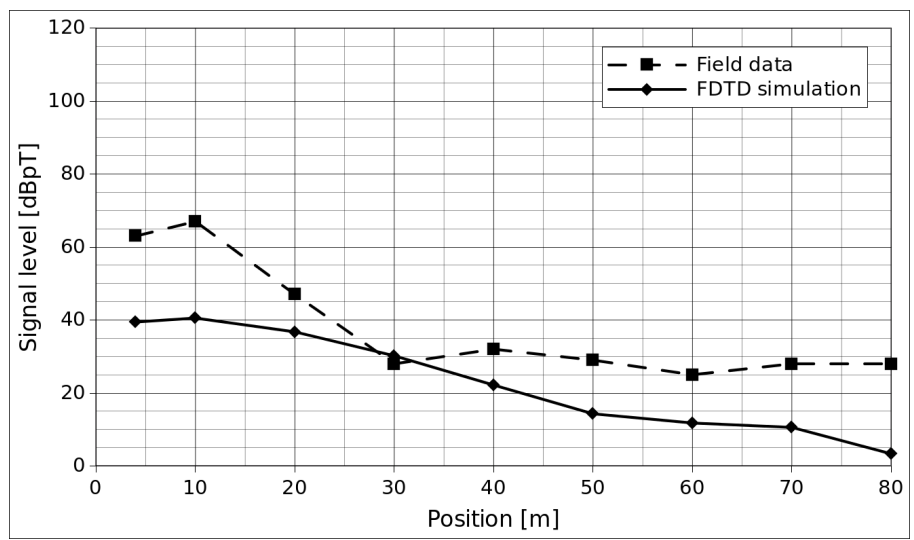

(b) $B_{y}$

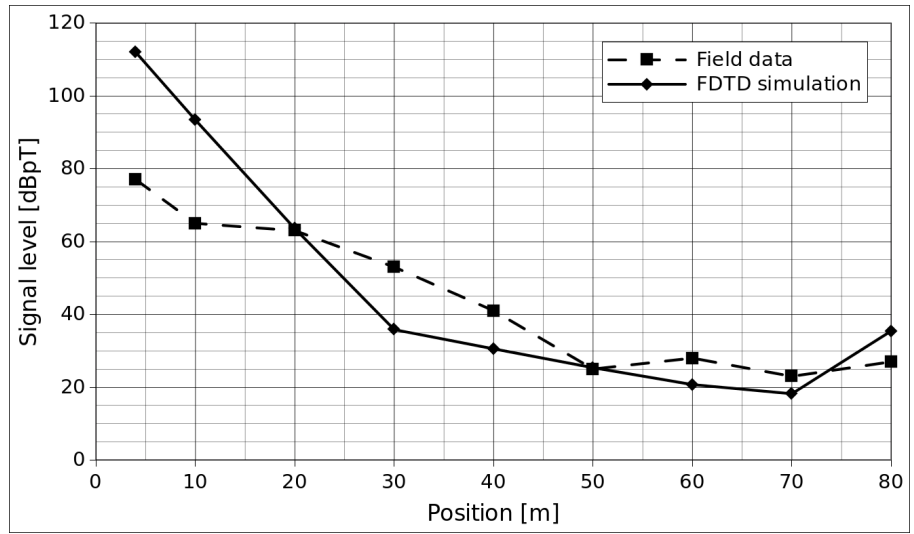

(c) $B_{z}$

Figure 6.2: Comparison between observed and modelled data for the $B_{x}$ (a), $B_{y}$ (b), and $B_{z}$ (c) components one level below the roof of the parking garage. The position of the receiver is measured along the $\mathrm{x}$-axis relative to the position of the transmitter. 
is more difficult to interpret. Without the presence of the building, $B_{y}$ would be expected to be null as the receivers are aligned along $y=0$; therefore, even small uncertainties on the order of $5-10 \mathrm{~cm}$ in the y position result in considerable variations in $B_{y}$. For this reason, and due to the difficulty of resolving the expected $B_{y}$ signal in the noisy environment, the match between the observed and modelled $B_{y}$ signal strength is not as consistent as for $B_{x}$ and $B_{z}$.

Two-dimensional slices (xz plane) of signal strength for the $B_{x}, B_{y}$, and $B_{z}$ components are given in Figure 6.3. An important observation seen in $B_{z}$-both in the observed and modelled data - is that there is an increase in signal strength near the edge of the building away from the transmitter. While moving away from the transmitter, the $B_{z}$ signal dives below the noise threshold, but when the receiver approaches the far edge of the building, the $B_{z}$ signal is once again above the noise threshold and can be received. Overall, the $B_{x}$ component of the field data is the strongest, especially far away from the transmitter, although $B_{z}$ is above the noise threshold in areas directly below the transmitter.

\subsubsection{Hotel}

The next example comes from a military base. One of the training buildings is built to resemble a hotel. It is five stories high and constructed of reinforced concrete. An elevator shaft goes down the building, but it does not contain an actual elevator and is instead sealed off by metal sheets. A transmitter was placed outside and centered about the building (Figure 6.4). The transmitter current was reduced to $20 \%$ (i.e. to $2 \mathrm{~A}$ ) to avoid receiver saturation. Measurements were made along the transmitter axis (x-axis), while going into the building.

Figure 6.5 compares the observed and modelled data for four levels above the 


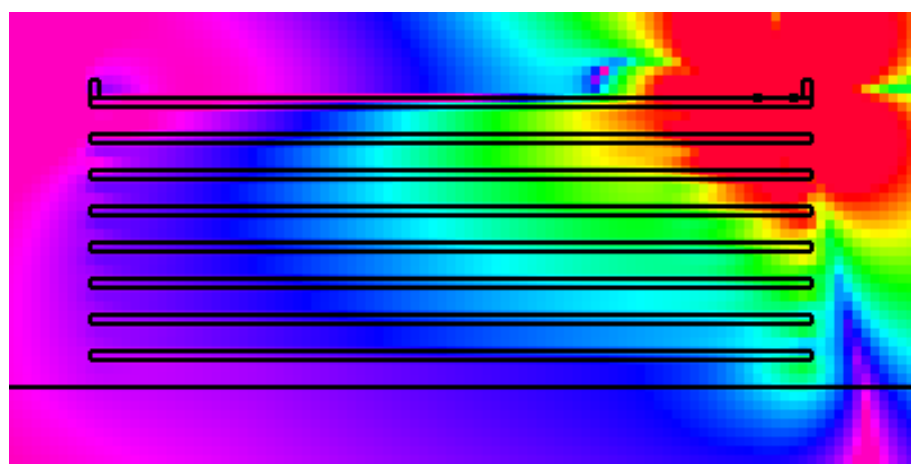

(a) $B_{x}$

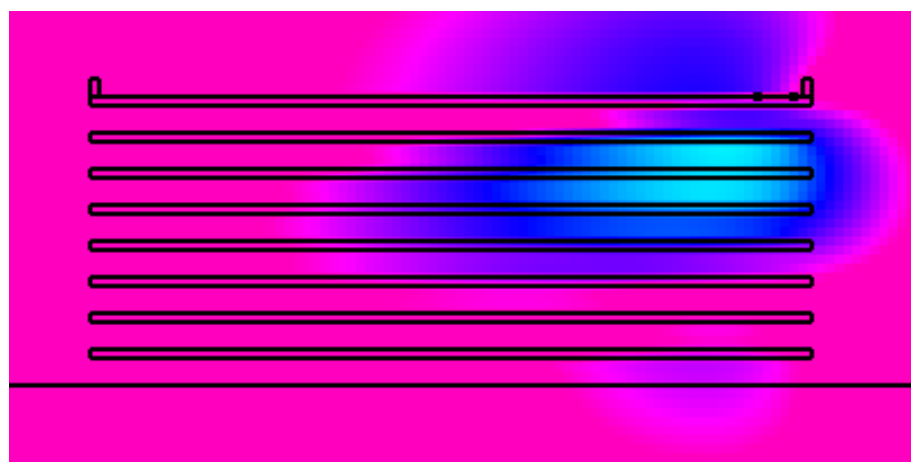

(b) $B_{y}$

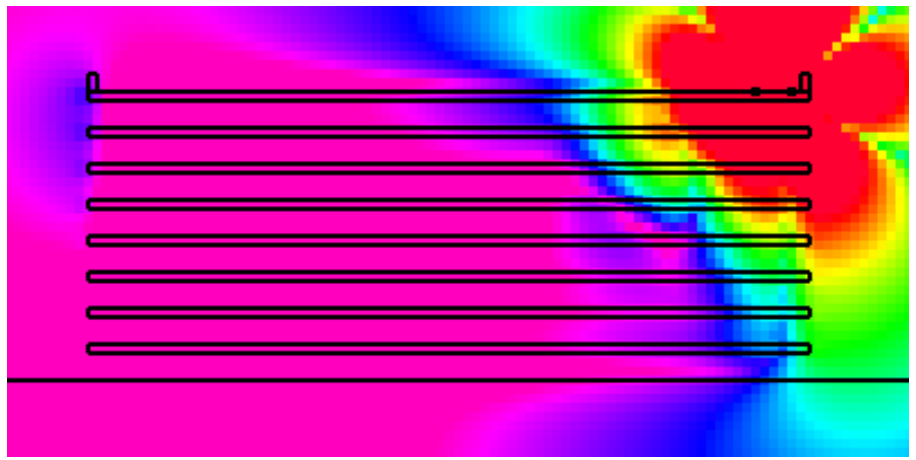

(c) $B_{z}$

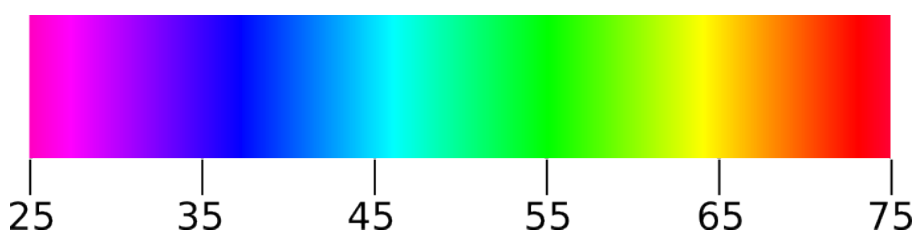

Figure 6.3: Modelled signal strength in dBpT for the $B_{x}(\mathrm{a}), B_{y}(\mathrm{~b})$, and $B_{z}$ (c) components in the parking garage. This view bisects the transmitter along the xz plane. The continuous black line represents the ground; the repeated rectangular segments represent floors. 


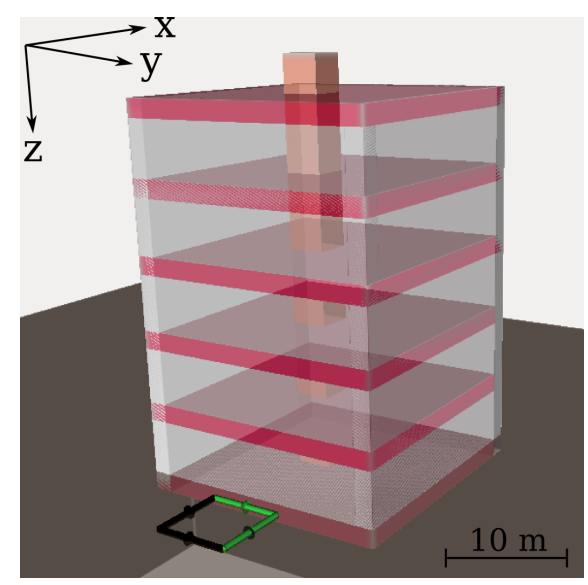

Figure 6.4: Model of hotel; the building footprint is $14 \mathrm{~m} \mathrm{x} 14 \mathrm{~m}$. The elevator shaft is represented by the orange bar running down the centre of the building.

ground. Conductances of $100 \mathrm{~S}$ and $10 \mathrm{~S}$ are used for the floors and walls, respectively. In the model, the elevator shaft is represented as a solid object of cross-section $2 \mathrm{~m} \times 2 \mathrm{~m}$. Its position corresponds between $5 \mathrm{~m}$ and $7 \mathrm{~m}$ along the profile (x-axis) and between $-1 \mathrm{~m}$ and $1 \mathrm{~m}$ to the side (y-axis). As there are rooms within the building, the direct line of sight to the transmitter is compromised; therefore, modelled results have been calculated for five possible positions along the y-axis.

As a general trend, the $B_{x}$ and $B_{z}$ components consistently decrease while moving away from the transmitter. The presence of the elevator shaft results locally in a greater decay rate than for other positions. In both the observed and modelled data, the $B_{z}$ component is more greatly affected by the elevator shaft than the $B_{x}$ component. As was seen in the parking garage, there is an increase in signal strength in the $B_{z}$ component when approaching the edge of the building away from the transmitter. In all three components, the strong nulls for the $\mathrm{y}=0.0 \mathrm{~m}$ and $\mathrm{y}=0.5 \mathrm{~m}$ profiles, centered at $\mathrm{x}=6 \mathrm{~m}$, result from sampling in the modelled elevator shaft, which is not representative of the field data. Based on the five different y-positions, the zigzagged nature of the observed $B_{y}$ data can be explained by uncertainties in the position of the receiver along the y-axis, as previously noted in the parking garage 


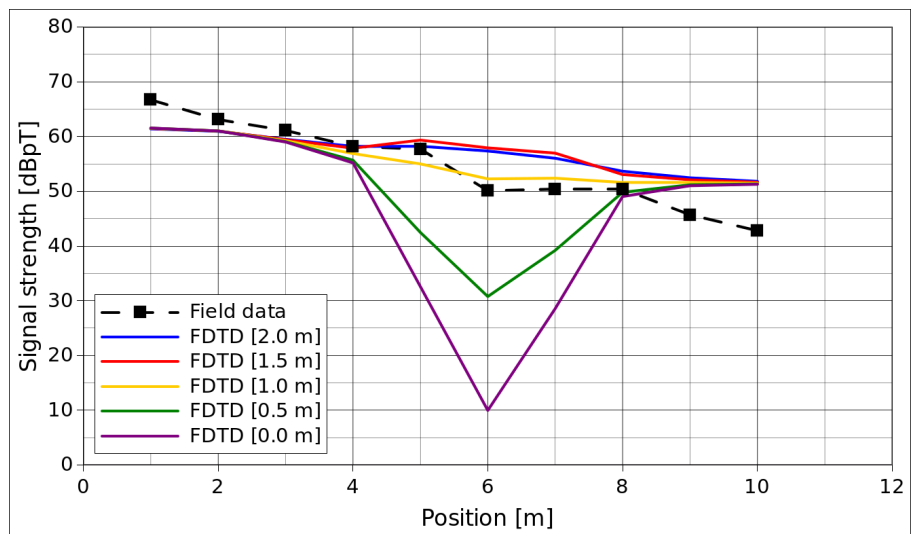

(a) $B_{x}$

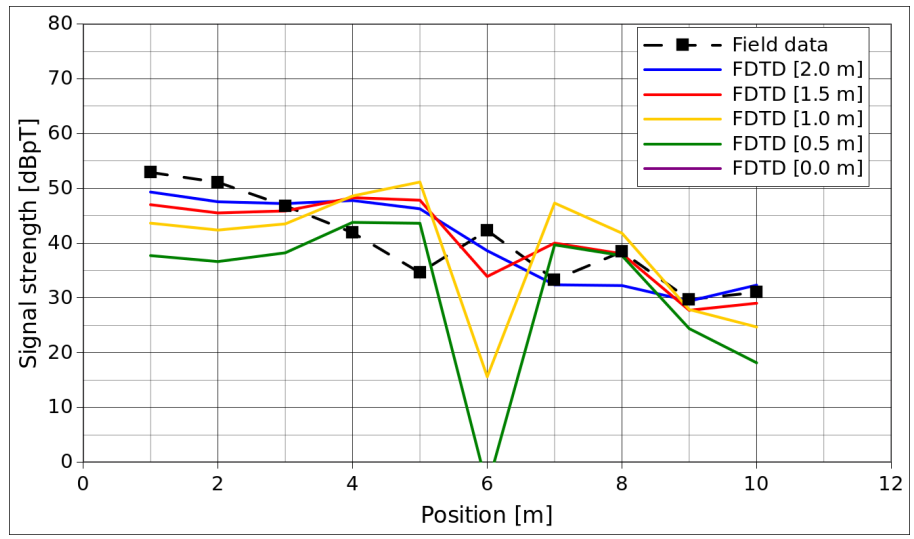

(b) $B_{y}$

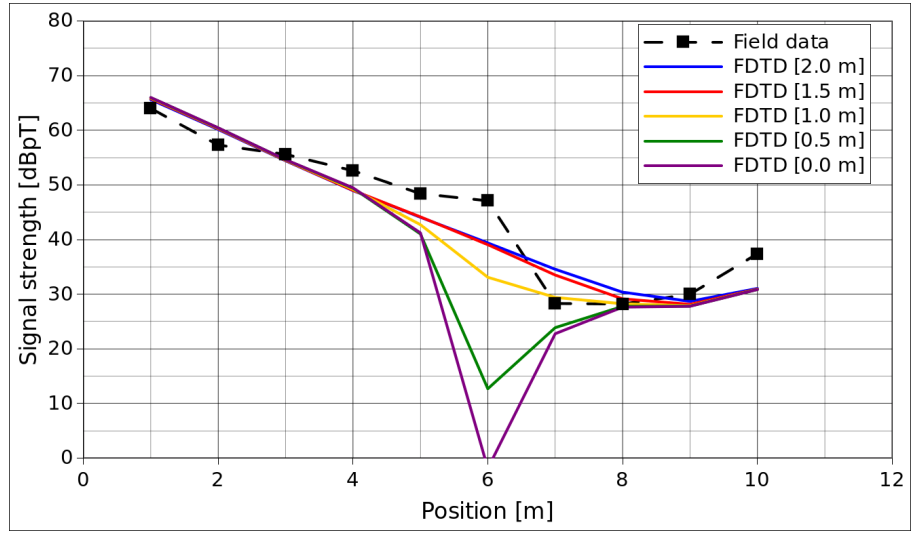

(c) $B_{z}$

Figure 6.5: Comparison between observed and modelled data for the $B_{x}$ (a), $B_{y}$ (b), and $B_{z}$ (c) components along four levels above the ground in the hotel. The position of the receiver is measured along the $\mathrm{x}$-axis relative to the position of the transmitter. The modelled profiles are given for five positions, from $y=$ $0.0 \mathrm{~m}$ to $\mathrm{y}=2.0 \mathrm{~m}$. 


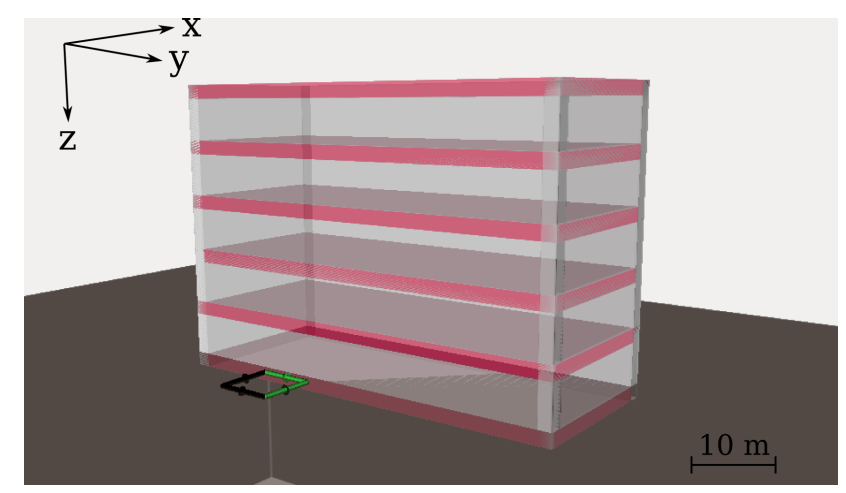

Figure 6.6: Model of embassy; the building footprint is $30 \mathrm{~m} \mathrm{x} 12 \mathrm{~m}$.

example.

\subsubsection{Embassy}

The embassy is another training building at the military base (Figure 6.6). An important difference from the two previous examples is that the concrete floors of the embassy are reinforced with steel sheets, which has a conductance greater than that of concrete reinforced with rebar. The transmitter was placed outside the building, and offset from the centre of the building by approximately $5 \mathrm{~m}$. As for the hotel, the transmitter current was reduced to $20 \%$ (2 A) to avoid saturation.

Figure 6.7 compares the observed and modelled data for one level above the ground. As was done for the hotel, five possible profiles were modelled to account for the sensitivity of the $B_{y}$ component to position along the y-axis. Conductances of $1000 \mathrm{~S}$ for the floor and $100 \mathrm{~S}$ for the walls were used.

At $\mathrm{x}=8 \mathrm{~m}$, there is a modelled null in the $B_{z}$ component. While there is a decrease seen about the same position in the field data, it is not as sharp as in the modelled data, due to a noise level of $20 \mathrm{dBpT}$. At distances greater than $\mathrm{x}=8 \mathrm{~m}$, the increase in $B_{z}$ could be attributed to the receiver approaching the edge of the building, as was observed in the parking garage and hotel examples. Unlike the $B_{x}$ 


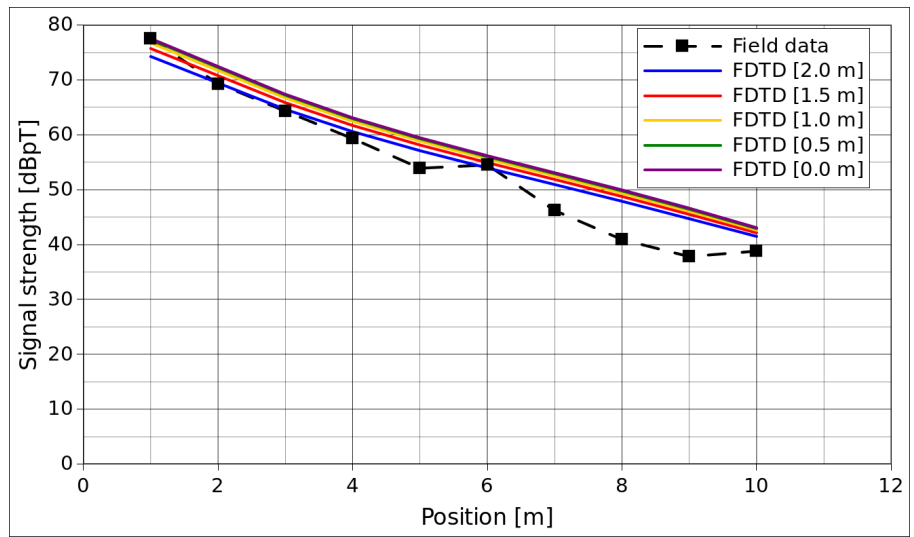

(a) $B_{x}$

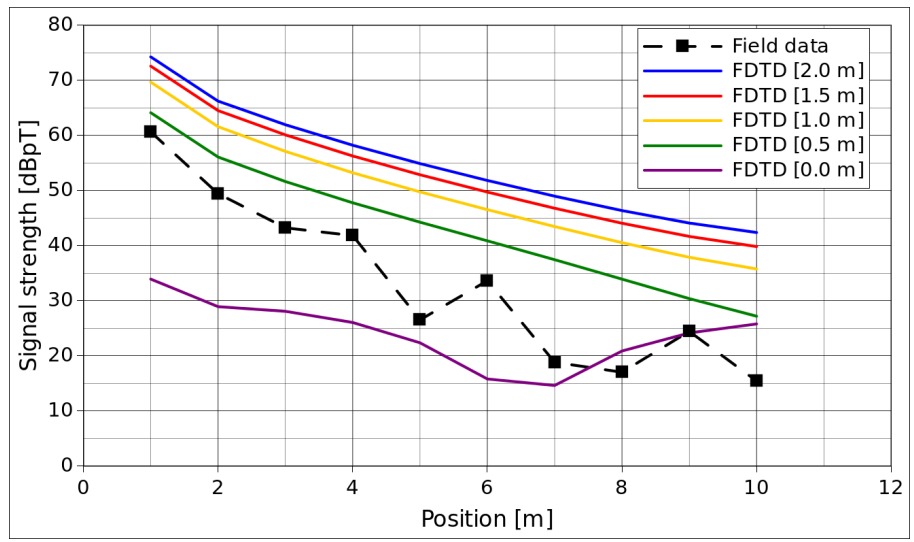

(b) $B_{y}$

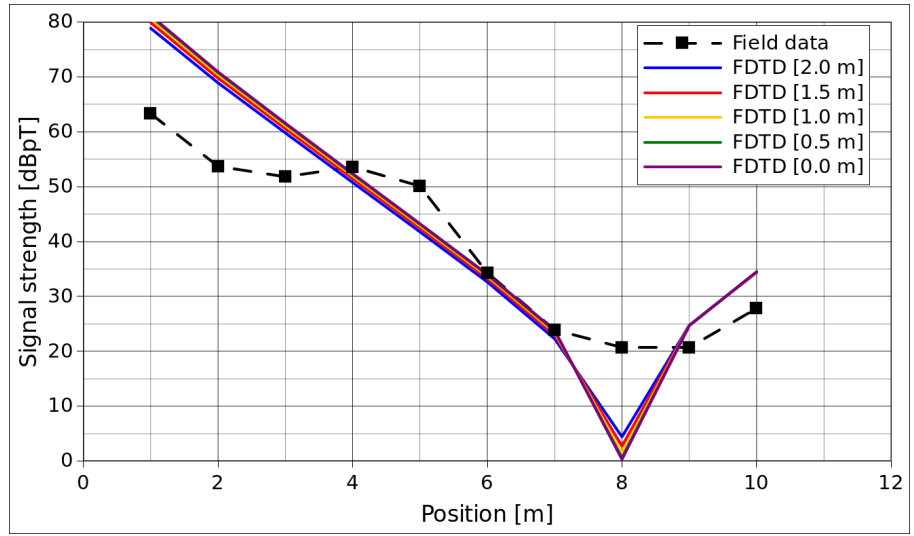

(c) $B_{z}$

Figure 6.7: Comparison between observed and modelled data for the $B_{x}$ (a), $B_{y}$ (b), and $B_{z}$ (c) components one level above the ground in the embassy. The position of the receiver is measured along the $\mathrm{x}$-axis relative to the position of the transmitter. The modelled profiles are given for five positions, from $\mathrm{y}=$ $0.0 \mathrm{~m}$ to $\mathrm{y}=2.0 \mathrm{~m}$. 


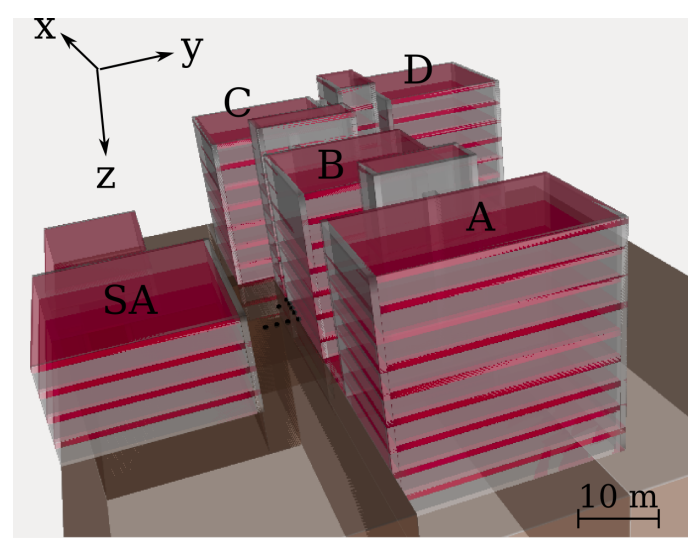

(a) Side view

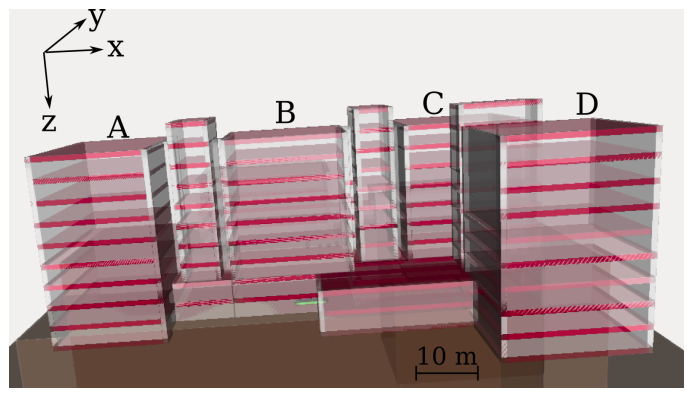

(b) Front view

Figure 6.8: Model of the Loeb Building. The entire complex spans $100 \mathrm{~m}$. The towers in Loeb Building are labelled from A to D; the neighbouring Southam Hall is labelled "SA".

and $B_{z}$ components, the $B_{y}$ component has an irregular profile shape. As also shown in the parking garage and hotel examples, the behaviour of the $B_{y}$ component is attributable to its sensitivity to position along the y-axis.

\subsubsection{Loeb Building}

The Loeb Building at Carleton University is composed of four linked nine-story towers (Figure 6.8). The front side is entirely above ground whereas the back side has two levels underground. The transmitter was placed on the ground level of Tower B, close to the front wall (i.e. farther away from Southam Hall), and measurements were made on road between the Loeb Building and Southam Hall (two levels above). Unlike in the three previous examples, below-ground to above-ground transmissions were made, thus the ground conductivity needed to be accounted for. Conductances of $35 \mathrm{~S}$ and 3.5 S were fitted for the floors and walls respectively; a ground conductivity of $0.01 \mathrm{~S} / \mathrm{m}$ was used.

Figure 6.9 compares the observed and modelled data for the $B_{x}$ and $B_{z}$ components. The strength of the $B_{y}$ component was below the $25 \mathrm{dBpT}$ noise threshold. 


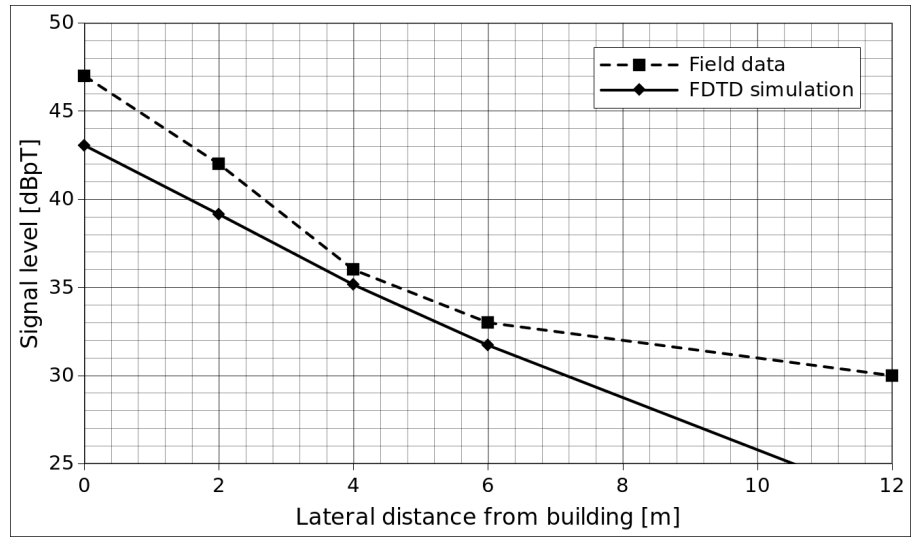

(a) $B_{x}$

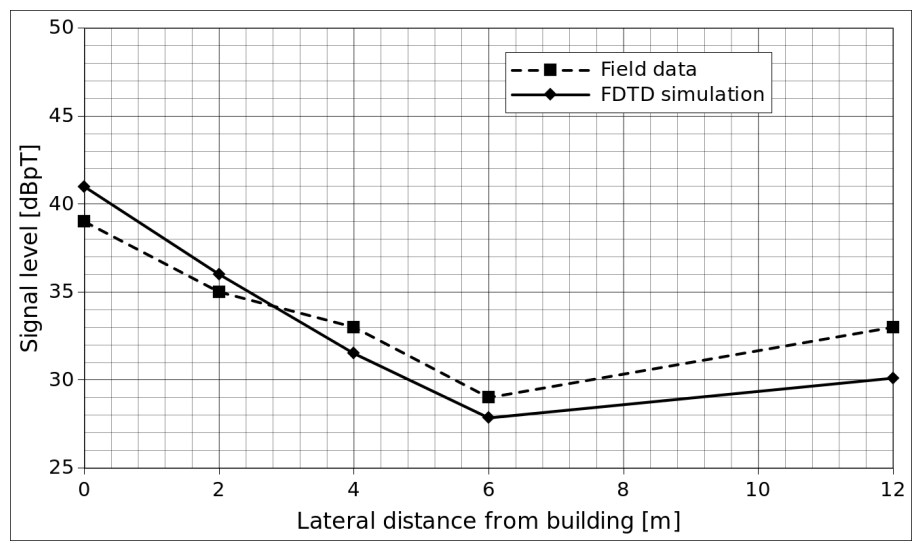

(b) $B_{z}$

Figure 6.9: Comparison between observed and modelled data for the $B_{x}$ (a), $B_{y}$ (b), and $B_{z}$ (c) components between Loeb Building and Southam Hall. The position of the receiver is measured along the $\mathrm{y}$-axis relative to the position of the transmitter.

An increase in $B_{z}$ signal strength when approaching Southam Hall $(\mathrm{x}=12 \mathrm{~m})$ is apparent in both datasets; in contrast, at that position, the $B_{x}$ signal was too weak to be detected above the noise. Based on modelling, a higher ground conductivity resulted in greater attenuation; the conductivity value chosen, $0.01 \mathrm{~S} / \mathrm{m}$, is typical for soil (Keller, 1988).

Two-dimensional slices (xy plane) of signal strength for the $B_{x}, B_{y}$, and $B_{z}$ components are given in Figure 6.10. In $B_{z}$ component, there is a null outside tower $\mathrm{B}$, visible as a semicircular feature of signal under $20 \mathrm{dBpT}$. Outside the building, the 


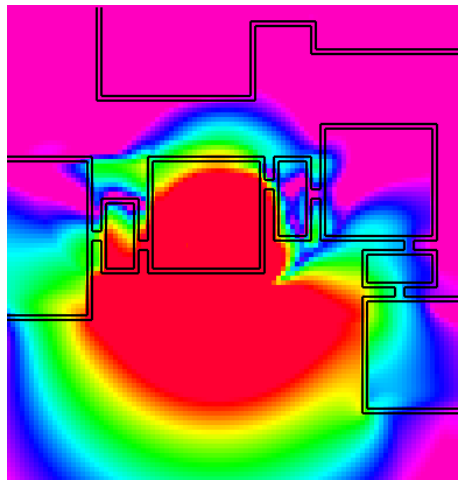

(a) $B_{x}$

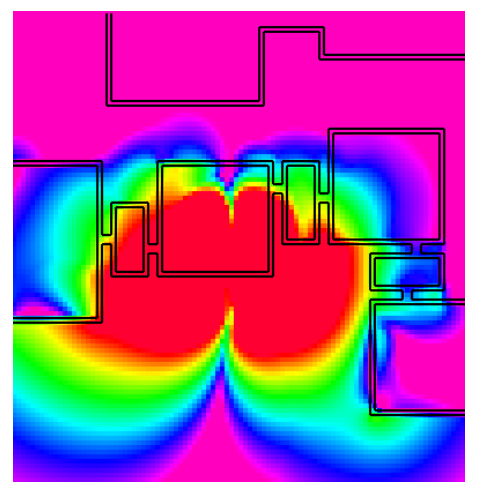

(b) $B_{y}$

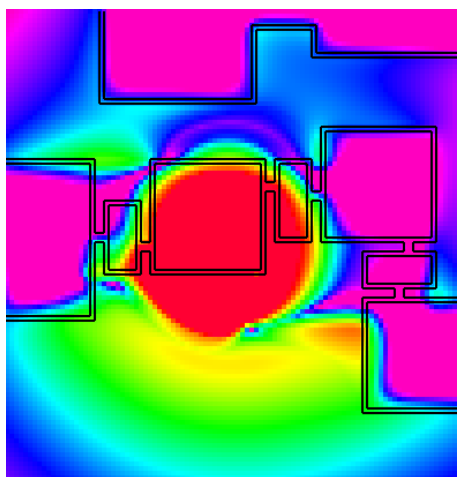

(c) $B_{z}$

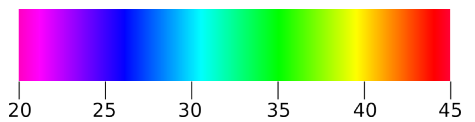

Figure 6.10: Modelled signal strength in dBpT for the $B_{x}(\mathrm{a}), B_{y}(\mathrm{~b})$, and $B_{z}$ (c) components in Loeb Building. The towers are seen from left to right as A to D. A bird's-eye view at two levels above the ground is shown.

$B_{y}$ component is largely unresolvable in the noisy environment. Both of these observations also apply to the observed data. In the area between Towers $\mathrm{A}$ and $\mathrm{B}$, the $B_{x}$ and $B_{z}$ components are particularly elevated. This location was revisited and the presence of elevated signal was confirmed, thereby demonstrating the predictive power of the FDTD models. Finally, the effect of the ground can be seen. In the upper part of the slice (relative to the page), the signal propagates through the equivalent of two levels of soil, whereas in the bottom part, it goes through air. Consequently, the signal strength in the bottom part of the slice is much greater than in the upper part.

\subsection{Discussion}

In the parking garage, hotel, and embassy examples, for both the observed and modelled data, there is an increase in the $B_{z}$ component when approaching, from inside, the building edge furthest from the transmitter. Such a result is not intuitive: when 
moving further away from the transmitter, it is expected that the signal will generally display a decreasing trend (e.g. for a vertical magnetic dipole, even if $B_{z}$ does not monotonically decrease with distance, the overall trend is that of decay). The physical explanation for this effect stems from the placement of the transmitter outside the building - in the case study where the transmitter was inside the building (Loeb Building), the effect is not observed. Part of the magnetic field is present outside the building and does not get attenuated by conductors. For example, the $B_{x}$ and $B_{z}$ components in the parking garage (Figures $6.2 \mathrm{a}$ and $6.2 \mathrm{c}$ respectively) maintain the pattern characteristic of the field of a magnetic dipole in free space. If magnetic field lines are considered, those at the edge of the building would interact less with conductors than those inside the building. This explanation suggests that for the locations studied, both $B_{x}$ and $B_{z}$ would increase ( $B_{y}$ is presumed to be mostly null), but only the $B_{z}$ signal is affected. This discrepancy is explained by the orientation of the transmitter. A horizontal loop will largely excite eddy currents in the horizontal plane, which results in stronger attenuation of the $B_{z}$ component in the presence of conductors as opposed to the $B_{x}$ component. As the $B_{x}$ component is more attenuated by geometric spreading, its strength will not increase at the edge as the degree of attenuation due to propagation in a conductive medium remains comparable. An important practical implication of this result is that more of the building can be covered by placing the transmitter outside it (including on the roof).

In the Loeb Building, a localized zone of strong $B_{x}$ and $B_{z}$ signals was identified between Towers $\mathrm{A}$ and $\mathrm{B}$ in simulations done during analysis; the presence of this zone was later experimentally verified. The presence of the area of strong signal outside the building points to an explanation similar to the previous example, as part of the signal would propagate through the more resistive soil as opposed to the reinforced concrete floors. In this example, the signal propagates through a 
conductive ground, which would account for the distortion of the $B_{x}$ component, which is not similarly observed in the other case studies, which all had above-ground transmitters. These results further show that a conductive ground will contribute to the attenuation of the fields when transmitting from, or receiving in an underground locations, even in the presence of significantly more conductive structures. To fit the observed data, a best-fitting ground conductivity had to be assigned in addition to the floor and wall conductances. When setting up a TTE radio link where one location is underground, the conductivity structures of the subsurface (i.e. geological controls) are of equal importance as is the structure of the building. By positioning the transmitter as to orient the field lines through more resistive media (i.e. in the case of the Loeb Building, the ground), the signal strength at the receiver can be improved. This approach would also help mitigate the challenge of two-way communications, as transmitters located outside can cover more of the building interior as compared to the coverage outside a building from a transmitter located inside.

Both the observed and modelled data show that unusually conductive large objects will distort and further attenuate the signal. The signal decay rate with distance away from the transmitter was markedly greater in the embassy (40 dBpT / $10 \mathrm{~m}$ ), which had steel floors, compared to the hotel (25 dBpT / $10 \mathrm{~m})$. While the presence of steel floors were unavoidable in the embassy, it was nevertheless possible to receive a signal in this challenging environment. In the hotel, when the receiver was placed behind the elevator shaft closed off with thick steel doors, the signal sharply dropped. When unusual conductive structures are known to exist, the transmitter-receiver pair could be positioned to avoid the conductor. 


\subsection{Conclusion}

Experimental data for near-field VLF TTE radio signal propagation were acquired in four multistory buildings with different structures, for a variety of transmitterreceiver setups. Conductance models were fitted to the observed data; conductance values ranging from 30-100 S were obtained for reinforced concrete floors and a steel floor was found to have a conductance of $1000 \mathrm{~S}$. The conductance values of the floors were an order of magnitude greater than those of the walls. This result can be explained by the reduced sensitivity of a horizontal loop to vertical structures, or by less reinforcements and more discontinuities in walls as compared to floors. Unexpected observations were replicated with the FDTD simulations, and vice-versa. An observed increase in the $B_{z}$ component at the edge of a building furthest from the transmitter was replicated in the models; alternatively, in the Loeb Building experiment, a zone of higher signal strength was first seen in a model, and later verified in the field. The measurement of all three components helped to formulate physical explanations for the observed magnetic field strengths; however, as most measurements were done along a transmitter axis (i.e. $y=0$ ), the corresponding component $\left(B_{y}\right)$ was highly sensitive to small changes in position, which made it more difficult to fit a model to those observations.

The agreement between observed and modelled data validates the modelling ap-

proach. Forward models can be used to assess the viability of, or optimize a TTE radio communication link in multistory buildings. Models can be used to find zones of unexpectedly strong or weak signal; such information could in turn guide the placement of the transmitter and receiver to take advantage of high signal strength. Alternatively, the transmitter and receiver could be positioned as to mitigate the additional attenuation caused by a conductor particular to a given building. 


\subsection{Acknowledgments}

We thank Carling Walsh for her assistance with the field work. This research project is funded by the Natural Sciences and Engineering Research Council of Canada (NSERC) and Vital Alert Communication, Inc., through an Industrial Postgraduate Scholarship to M. Ralchenko. 


\section{Chapter 7}

\section{Conclusion}

\section{$7.1 \quad$ Summary}

This thesis addressed the problem of accurately modelling through-the-Earth (TTE) radio transmissions. Two broad areas were investigated: first, to develop an efficient forward modelling tool to simulate TTE radio transmissions in inhomogeneous media,

and second, to use the TTE radio signals to estimate a site-specific conductivity structure.

To model TTE radio transmissions, a finite-difference time-domain (FDTD) code was written. The FDTD method handles inhomogeneous media by discretizing the geometrical domain into a rectangular grid. To improve performance, the FDTD code was parallelized on a general-purpose graphics processing unit (GPU), which permitted most simulations to be executed in a matter of minutes to hours. The FDTD code was validated against three analytic solutions.

The use of a TTE radio signal to estimate subsurface conductivity was demonstrated at an experimental coal mine. By measuring the signal strength underground, transmitted from an above-ground terminal, at several locations and several frequencies, a four-layer conductivity model, with topography, was fitted. When combined 
with further modelling, a site-specific conductivity structure is a powerful piece of information. In a mine disaster, communication would be attempted between a belowground unit (BGU) and an above-ground unit (AGU). While the position of the BGU is more constrained (e.g. refuge chamber), the AGU could be optimally positioned based on forward models. Additionally, an ideal operating frequency could be chosen or the maximum operating range could be estimated, via accurate forward modelling.

Two situations where TTE radio could be used were investigated experimentally and numerically. TTE radio signals have been previously observed to couple to elongated conductors, such as railway tracks in underground mines or civilian tunnels, or cables and frames in elevator shafts, thereby increasing signal range; however, no systematic study of this effect was previously undertaken. The existing FDTD code was upgraded to handle such conductors as wires thin relative to the cell size. Measurements were done at railway tracks and next to an elevator shaft in a 22-story building to gather experimental data that validated the model. It was shown that the induction of currents in these conductors allows a TTE radio operator to improve signal range by exploiting the presence of these structures. Further work was done on modelling multistory buildings; the propagation of a near-field VLF signal in multistory buildings had not been previously studied. By modelling the floors and walls as sheets of a given conductance, experimental data were reconciled with the calculated data. This approach was validated in a variety of scenarios, including different transmitter and receiver locations inside or outside the building, in a building with steel floors, and for a building that was partially underground. When modelling these new settings, conductances were fitted to the observed data using the FDTD code. By being able to both accurately model signal strength, and estimate the underlying conductivity structure, zones of strong or weak signal strength can be constrained, which would guide the placement of the transmitter and receiver in the field to obtain 
a viable TTE radio communications link.

\subsection{Future work}

The problem of modelling TTE radio transmissions, and the associated problem of estimating the conductivity distribution in the context of TTE radio transmissions, were addressed in this thesis by developing a FDTD-based forward modeller, and applying it to model signal strength and estimate to conductivity structures in various propagation environment. Nevertheless, there remains several avenues of additional work on modelling TTE radios. Different environments pose unique constraints on the propagation of a near-field VLF signal. While this thesis considered several environments, namely coal mines, multistory buildings, and those having elongated conductors, there are many other situations and environments in which this technology could be applicable. For example, the presence of fire could affect the signal. Or, the materials used in a building could differ; only reinforced concrete constructions were considered in this thesis. Additionally, using different frequency bands, such as medium frequency (MF; 300-3000 kHz), could be considered, as it can encode more information than a VLF signal, and it can exploit transmission line effects in the presence of elongated conductors.

An alternative area for future work on the subject is to refine the forward modelling code. The FDTD algorithm used in this work is conditionally stable, which imposed a constraint on the maximum permissible time step. Advances have been made in unconditionally stable algorithms which permit a larger time step, but further research is needed to improve accuracy at large time steps and in making the algorithm more parallelizable, which is a challenge compared to conventional FDTD. 


\section{Bibliography}

Adveev, D., 2005, Three-dimensional electromagnetic modelling and inversion from theory to application: Surveys in Geophysics, 26, 769-799.

Ames, L., J. DeBettencourt, J. Frazier, and A. Orange, 1963, Radio communications via rock strata: IEEE Transactions on Communications, 11, 159-169.

Austin, A. C. M., 2016, Wireless channel characterisation in burning buildings over 100-1000 MHz: IEEE Transactions on Antennas and Propagation, 64, 3265-3269.

Austin, A. C. M., M. J. Neve, and G. B. Rowe, 2011, Modeling propagation in multifloor buildings using the FDTD method: IEEE Transactions on Antennas and Propagation, 59, 4239-4246.

Ayuso, N., J. A. Cuchi, F. Lera, and J. L. Villarroel, 2010, Accurately locating a vertical magnetic dipole buried in a conducting Earth: IEEE Transactions on Geoscience and Remote Sensing, 48, 3676-3685.

Baba, Y., N. Nagaoka, and A. Ametani, 2005, Modeling of thin wires in a lossy medium for FDTD simulations: IEEE Transactions on Electromagnetic Compatibility, 47, 54-60.

Barret, W. M., 1952, Note on the radio transmission demonstration at Grand Saline, Texas: Geophysics, 17, 544-549.

Beggs, J. H., R. J. Luebbers, K. S. Yee, and K. S. Kunz, 1992, Finite-difference timedomain implementation of surface impedance boundary conditions: IEEE Transactions on Antennas and Propagation, 40, 49-56.

Bergmann, T., J. Robertsson, and K. Holliger, 1998, Finite-difference modeling of electromagnetic wave propagation in dispersive and attenuating media: Geophysics, 63, 856-867.

Blaunstein, N., and M. Levin, 1996, VHF/UHF wave attenuation in a city with regularly spaced buildings: Radio Science, 31, 313-323.

Bridges, G. E. J., 1992, Fields generated by bare and insulated cables buried in a lossy 
half-space: IEEE Transactions on Geoscience and Remote Sensing, 30, 140-146.

Brocker, D., P. Sieber, J. Waynert, J. Li, P. Werner, and D. Werner, 2015, A hybrid approach for efficient modeling of medium-frequency propagation in coal mines: IEEE Antennas and Propagation Magazine, 57, 164-176.

Carson, J. R., 1926, Wave propagation in overhead wires with ground return: Bell System Technical Journal, 5, 539-554.

Chang, D., and J. Wait, 1974, Extremely low frequency (ELF) propagation along a horizontal wire located above or buried in the earth: IEEE Transactions on Communications, 22, 421-427.

Coggeshall, E. J., E. W. Felegy, and H. J. Harrison, 1948, Some studies on emergency mine communications: Bureau of Mines.

Cyganski, D., J. Orr, and M. W. R., 2003, A multi-carrier technique for precision geolocation for indoor/multipath environments: 6th International Technical Meeting of the Satellite Division of The Institute of Navigation (ION GPS/GNSS 2003), 1069-1073.

Daily, W., and E. Owen, 1991, Crossborehole resistivity tomography: Geophysics, 56, 1228-1235.

Dalke, R. A., C. L. Holloway, P. McKenna, M. Johansson, and A. S. Ali, 2000, Effects of reinforced concrete structures on RF communications: IEEE Transactions on Electromagnetic Compatibility, 42, 486-496.

de la Kethulle de Ryhove, S., and R. Mittet, 2014, 3D marine magnetotelluric modeling and inversion with the finite-difference time-domain method: Geophysics, 79, E269-E286.

de la Kethulle de Ryhove, S., J. Morten, and K. Kumar, 2013, Efficient 3D MT inversion using finite-difference time-domain modelling: Presented at the 75 th EAGE Conference \& Exhibition incorporating SPE EUROPEC 2013.

Delogne, P., 1982, Leaky feeders and subsurface radio communication: Peter Perigrinus.

Deryck, L., 1974, Étude de la propagation des ondes électromagnétiques guidées le long d'une ligne bifilaire dans les galeries southerraines: Communauté europene du charbon et de l'acier.

Dokoupil, S., J. Karpinský, and M. Kašpar, 1962, The attenuation of electromagnetic waves in rocks: Studia Geophysica et Geodaetica, 6, 176-192.

Dukeshire, A., T. Tan, M. Okoniewski, and M. Potter, 2011, Marine CSEM scattered 
subsurface response detection using total-field scattered-field FDTD formulation: Presented at the Proceedings of the 5th European Conference on Antennas and Propagation (EUCAP).

Durkin, J., 1982, Apparent Earth conductivity over coal mines as estimated from through-the-Earth electromagnetic transmission tests: Bureau of Mines.

— $1984 \mathrm{a}$, Apparent Earth conductivity over coal mines as estimated from through-the-Earth electromagnetic transmission tests: Bureau of Mines.

$\longrightarrow, 1984 \mathrm{~b}$, Electromagnetic detection of trapped miners: IEEE Communications Magazine, 22, 37-46.

— 1991 , Earth conductivity estimates from through-the-Earth electromagnetic transmission tests: IEEE Transactions on Geoscience and Remote Sensing, 29, 300-307.

— 1997 , Surface vertical magnetic field produced by a finite loop buried in an earth containing a thin conducting sheet: Radio Science, 32, 19-23.

Elsherbeni, A., and V. Demir, 2009, The finite-difference time-domain method for electromagnetics with MATLAB simulations: SciTech Publishing.

Emslie, A. G., and R. L. Lagace, 1976, Propagation of low and medium frequency radio waves in a coal seam: Radio Science, 11, 253-261.

Enkin, R. J., D. Cowan, J. Tigner, A. Severide, D. Gilmour, A. Tkachyk, M. Kilduff, B. Vidal, and J. Baker, 2012, Physical property measurements at the GSC paleomagnetism and petrophysics laboratory, including electric impedance spectrum methodology and analysis, Geological Survey of Canada Open File 7227: Geological Survey of Canada.

Felegy, E. W., and E. J. Coggeshall, 1948, Applicability of radio to emergency mine communications: Bureau of Mines.

Forooshani, A., S. Bashir, D. Michelson, and S. Noghanian, 2013, A survey of wireless communications and propagation modeling in underground mines: IEEE Communications Surveys \& Tutorials, 15, 1524-1545.

Fritsch, V., 1963, Propagation of radiofrequency electromagnetic fields in geological conductors: Journal of Research of the National Bureau of Standards, Section D: Radio Propagation, 67D, 161.

Geyer, R., and G. Keller, 1976, Constraints affecting through-the-earth electromagnetic signalling and location techniques: Radio Science, 11, 323-342.

Geyer, R. G., G. V. Keller, and T. Ohya, 1974, Research on the transmission of 
electromagnetic signals between mine workings and the surface: Bureau of Mines.

Gibson, A., 2003, Channel characterization and system design for sub-surface communications: PhD thesis, University of Leeds, Leeds, UK.

Greene, F., 1967, The near-zone magnetic field of a small circular-loop antenna: Journal of Research of the National Bureau of Standards, Section C: Engineering and Instrumentation, 71C, 319-326.

Griffiths, D., 1999, Introduction to electrodynamics 3 ed.: Prentice Hall.

Haycock, O. C., E. C. Madsen, and S. R. Hurst, 1949, Propagation of electromagnetic waves in earth: Geophysics, 14, 162-171.

Hieda, I., and K. C. Nam, 2008, FDTD simulation of radio imaging method for biomedical application: 4th Kuala Lumpur International Conference on Biomedical Engineering 2008, 566-569.

Hill, D., and J. Wait, 1974, Diffusion of electromagnetic pulses into the earth from a line source: IEEE Transactions on Antennas and Propagation, 22, 145-146.

— 1982 , Theoretical noise and propagation models for through-the-earth communication: National Telecommunications and Information Administration, Institute for Telecommunication Sciences.

Hill, D. A., 1988, Magnetic dipole excitation of a long conductor in a lossy medium: IEEE Transactions on Geoscience and Remote Sensing, 26, 720-725.

—, 1990 , Magnetic dipole excitation of an insulated conductor of finite length: IEEE Transactions on Geoscience and Remote Sensing, 28, 289-294.

Hill, D. A., and J. R. Wait, 1977, Coupling between a dipole antenna and an infinite cable over an ideal ground plane: Radio Science, 12, 231-238.

Ilsley, L. C., H. B. Freeman, and D. H. Zellers, 1928, Experiments in underground communication through Earth strata, technical paper 433: Bureau of Mines.

Inman, M., A. Elsherbeni, J. Maloney, and B. Baker, 2007, GPU-based FDTD solver with CPML boundaries: Presented at the 2007 IEEE Antennas and Propagation International Symposium.

Irvine, S. E., 2010, Numerical simulation of wire detection using radio-frequency transmitter-receiver pairs: Technical report, Defence Research and Development Canada, Suffield, AB.

Janovský, L., 1987, Elevator mechanical design: principles and concepts: Ellis Horwood Limited. 
Karhunen, K., 2013, Electrical resistance tomography imaging of concrete: PhD thesis, University of Eastern Finland, Kuopio.

Keller, G. V., 1988, Rock and mineral properties, in Electromagnetic Methods in Applied Geophysics: Society of Exploration Geophysicists, Investigations in Geophysics No. 3, 12-51.

Lagace, R., J. Dobbie, and W. Hawes, 1980, Detection of trapped miner electromagnetic signals above coal mines: Bureau of Mines.

Large, D., L. Ball, and A. Farstad, 1973, Radio transmission to and from underground coal mines-theory and measurement: IEEE Transactions on Communications, 21, 194-202.

Lee, J., R. Lee, and A. Cangellaris, 1997, Time-domain finite-element methods: IEEE Transactions on Antennas and Propagation, 45, 430-442.

Li, J., J. Waynert, and B. Whisner, 2013, An introduction to a medium frequency propagation characteristic measurement method of a transmission line in underground coal mines: Progress In Electromagnetics Research B, 55, 131-149.

Li, J., J. A. Waynert, B. Whisner, and N. W. Damiano, 2014, Comparison of medium frequency propagation characteristics of a transmission line measured from both ends in a coal mine entry: 2014 IEEE Antennas and Propagation Society International Symposium (APSURSI), 255-256.

Li, J., B. Whisner, and J. Waynert, 2012, Measurements of medium frequency propagation characteristics of a transmission line in an underground coal mine: 2012 IEEE Industry Applications Society Annual Meeting, 1-8.

Li, Y., and R. S. Smith, 2015, Forward modeling of radio imaging (RIM) data with the Comsol RF module: Computers \& Geosciences, 85, 60-67.

Mäkinen, R. M., J. S. Juntunen, and M. A. Kivikoski, 2002, An improved thin-wire model for FDTD: IEEE Transactions on Microwave Theory and Techniques, 50, $1245-1255$.

Maloney, J. G., and G. S. Smith, 1992, The use of surface impedance concepts in the finite-difference time-domain method: IEEE Transactions on Antennas and Propagation, 40, 38-48.

McGehee, F. M., 1954, Propagation of radio frequency energy through the Earth: Geophysics, 19, 459-477.

McKenna, S. P., and J. R. McKenna, 2010, Modeling and analysis of the response of a triaxial, frequency-domain electromagnetic induction sensor to a buried linear 
conductor: Geophysics, 75, F1-F14.

McKenna, S. P., K. B. Parkham, L. J. Perren, and J. R. McKenna, 2013, Automatic detection of a subsurface wire using an electromagnetic gradiometer: IEEE Transactions on Geoscience and Remote Sensing, 51, 132-139.

Naus, H. W. L., 2013, Modeling of buried wire detection by radio-frequency electromagnetic waves: IEEE Geoscience and Remote Sensing Letters, 10, 160-164.

Newman, G., 2013, A review of high-performance computational strategies for modeling and imaging of electromagnetic induction data: Surveys in Geophysics, 35, 85-100.

Niu, J., and J. J. Simpson, 2012, On the air-sea boundary in transient marine CSEM detection modeling of subseafloor hydrocarbon reservoirs: IEEE Antennas and Wireless Propagation Letters, 11, 651-654.

Noda, T., and S. Yokoyama, 2002, Thin wire representation in finite difference time domain surge simulation: IEEE Transactions on Power Delivery, 17, 840-847.

Okada, T., and A. Iwai, 1988, Natural VLF radio waves: Research Studies Press.

Olsen, R., and A. Farstad, 1973, Electromagnetic direction finding experiments for location of trapped miners: IEEE Transactions on Geoscience Electronics, 11, 178185.

Parkhomenko, E., 1967, Electrical properties of rocks: Plenum Press.

Pittman, W., R. Church, and J. McLendon, 1985, Through-the-Earth electromagnetic trapped miner location system: a review: Bureau of Mines.

Pritchett, W. C., 1952, Attenuation of radio frequency waves through the Earth: Geophysics, 17, 193-217.

Pullen, M. W., 1953, Geologic aspects of radio wave transmission: Illinois State Geological Survey.

Qian, W., and D. E. Boerner, 1995, Electromagnetic modelling of buried line conductors using an integral equation: Geophysical Journal International, 121, 203-214.

Ralchenko, M., M. Roper, M. Svilans, and C. Samson, 2016, Coupling of very low frequency electromagnetic signals to long man-made conductors: SEG Technical Program Expanded Abstracts 2016, 2154-2158.

—_ 2017a, Coupling of very low frequency through-the-Earth radio signals to elongated conductors: accepted in IEEE Transactions on Antennas and Propagation.

Ralchenko, M., M. Svilans, C. Samson, and M. Roper, 2015, Finite-difference timedomain modelling of through-the-earth radio signal propagation: Computers \& 
Geosciences, 85, 184-195.

Ralchenko, M., M. Svilans, C. Samson, C. Walsh, and M. Roper, 2017b, Estimating subsurface conductivity while optimizing through-the-Earth radio communications using a wideband electromagnetic signal: accepted in Geophysics.

Rice, L. P., 1959, Radio transmission into buildings at 35 and $150 \mathrm{mc}$ : The Bell System Technical Journal, 38, 197-210.

Roden, J., and S. Gedney, 2000, Convolution PML (CPML): An efficient FDTD implementation of the CFS-PML for arbitrary media: Microwave and Optical Technology Letters, 27, 334-339.

Sheinker, A., B. Ginzburg, N. Salomonski, L. Frumkis, and B.-Z. Kaplan, 2014, Remote tracking of a magnetic receiver using low frequency beacons: Measurement Science and Technology, 25, 105101.

Shope, S., 1982, Electromagnetic surface fields due to a magnetic dipole buried in a three-layered Earth: Bureau of Mines.

Simpson, J. J., 2012, An established numerical method applied to geophysics: Eos, Transactions, American Geophysical Union, 93, 265-267.

Smith, A. A., 1978, Attenuation of electric and magnetic fields by buildings: IEEE Transactions on Electromagnetic Compatibility, EMC-20, 411-418.

Sogade, J., Y. Vichabian, A. Vandiver, P. Reppert, D. Coles, and F. Morgan, 2004, Electromagnetic cave-to-surface mapping system: IEEE Transactions on Geoscience and Remote Sensing, 42, 754-763.

Stefański, T., S. Benkler, N. Chavannes, and N. Kuster, 2013, OpenCL-based acceleration of the FDTD method in computational electromagnetics: International Journal of Numerical Modelling: Electronic Networks, Devices and Fields, 26, $355-365$.

Stefański, T., N. Chavannes, and N. Kuster, 2011, Multi-GPU accelerated finitedifference time-domain solver in Open Computing Language: Progress in Electromagnetics Research Symposium Online, 7, 71-74.

Stolarczyk, L., 1991, Emergency and operational low and medium frequency band radio communications system for underground mines: IEEE Transactions on Industry Applications, 27, 780-790.

Strakosch, G. R., 1998, The vertical transportation handbook: John Wiley \& Sons, Inc.

Szarka, L., 1988, Geophysical aspects of man-made electromagnetic noise in the 
earth-a review: Surveys in Geophysics, 9, 287-318.

Taflove, A., and S. Hagness, 2005, Computational electrodynamics: the finitedifference time-domain method: Artech House.

Thomson, S., and S. Hinde, 1993, Bringing geophysics into the mine: radio attenuation imaging and mine geology: Exploration Geophysics, 24, 805-810.

Toivanen, J., T. Stefański, N. Kuster, and N. Chavannes, 2011, Comparison of CPML implementations for the GPU-accelerated FDTD solver: Progress in Electromagnetics Research M, 19, 61-75.

Tripp, A., R. McNearny, and C. Furse, 2000, Prof. James R. Wait and mining production technology-an appreciation: IEEE Transactions on Antennas and Propagation, 48, 1438-1441.

Umashankar, K., A. Taflove, and B. Beker, 1987, Calculation and experimental validation of induced currents on coupled wires in an arbitrary shaped cavity: IEEE Transactions on Antennas and Propagation, 35, 1248-1257.

Vogt, D., and A. Marvin, 2006, Characterising borehole radio antenna performance using finite-difference time-domain modelling: South African Journal of Geology, 109, 561-570.

Wait, J., 1954, Mutual coupling of loops lying on the ground: Geophysics, 19, 290296.

— 1970, Electromagnetic waves in stratified media 2 ed.: Pergamon Press.

, 1971, Electromagnetic probing in geophysics: The Golem Press.

—, 1975, Theory of EM wave propagation through tunnels: Radio Science, 10, $753-759$.

— 1982, Geo-electromagnetism: Academic Press.

$\longrightarrow$, 1986, Introduction to antennas and propagation: Peter Peregrinus.

Wait, J., and D. Hill, 1977, Radio frequency transmission via a trolley wire in a tunnel with a rail return: IEEE Transactions on Antennas and Propagation, 25, 248-253.

Wait, J., and K. Spies, 1972, Subsurface electromagnetic fields of a circular loop of current located above ground: IEEE Transactions on Antennas and Propagation, 20, 520-522.

Wait, J. R., 1978, Excitation of currents on a buried insulated cable: Journal of Applied Physics, 49, 876-880.

Wait, J. R., and K. R. Umashankar, 1978, Analysis of the earth resistivity response of buried cables: Pure and Applied Geophysics, 117, 711-742. 
Wang, T., and G. W. Hohmann, 1993, A finite-difference, time-domain solution for three-dimensional electromagnetic modeling: Geophysics, 58, 797-809.

Watt, A., 1967, VLF radio engineering: Pergamon Press.

Watts, R. D., 1978, Electromagnetic scattering from buried wires: Geophysics, 43, 767-781.

Williams, H., 1951, Subterranean communication by electric waves: Journal of the British Institution of Radio Engineers, 11, 101-111.

Wynn, J. C., and K. L. Zonge, 1975, EM coupling, its intrinsic value, its removal and the cultural coupling problem: Geophysics, 40, 831-850.

Yan, L., 2014, Measurements and modeling of the electromagnetic fields in throughthe-earth wireless communications: PhD thesis, University of New Mexico, Albequerque.

Yan, L., J. Waynert, and C. Sunderman, 2013, Measurements and modeling of through-the-Earth communications for coal mines: IEEE Transactions on Industry Applications, 49, 1979-1983.

— , 2014, Earth conductivity estimation from through-the-Earth measurements of 94 coal mines using different electromagnetic models: Applied Computational Electromagnetics Society Journal, 29, 755-762.

Yarkan, S., S. Guzelgoz, H. Arslan, and R. Murphy, 2009, Underground mine communications: a survey: IEEE Communications Surveys \& Tutorials, 11, 125-142.

Yee, K., 1966, Numerical solution of initial boundary value problems involving Maxwell's equations in isotropic media: IEEE Transactions on Antennas and Propagation, 14, 302-307.

Yenchek, M., G. Homce, N. Damiano, and J. Srednicki, 2012, NIOSH-sponsored research in through-the-Earth communications for mines: a status report: IEEE Transactions on Industry Applications, 48, 1700-1707.

Yu, L., M. Chouteau, D. E. Boerner, and J. Wang, 1998, On the imaging of radiofrequency electromagnetic data for cross-borehole mineral exploration: Geophysical Journal International, 135, 523-541. 